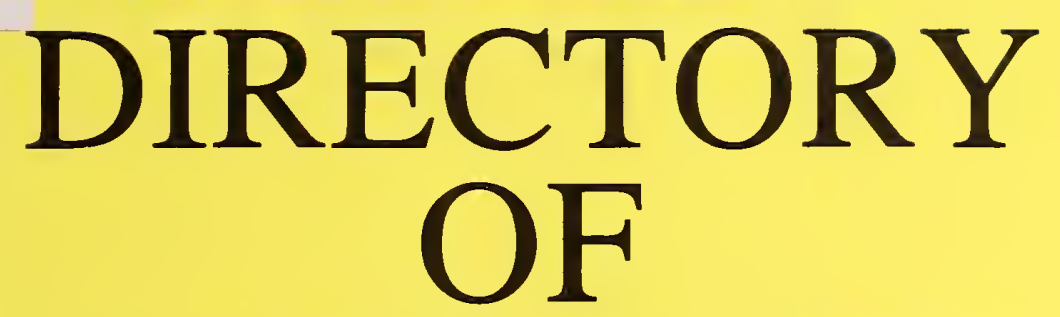

ASSOCIATE

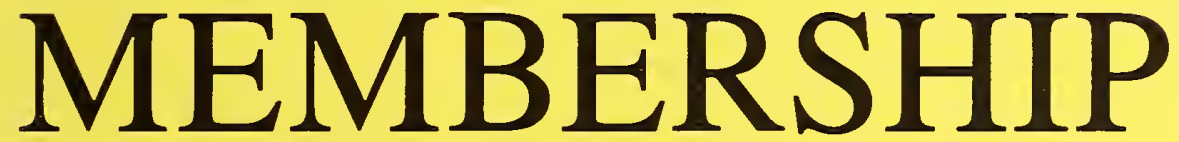

\title{
1986
}

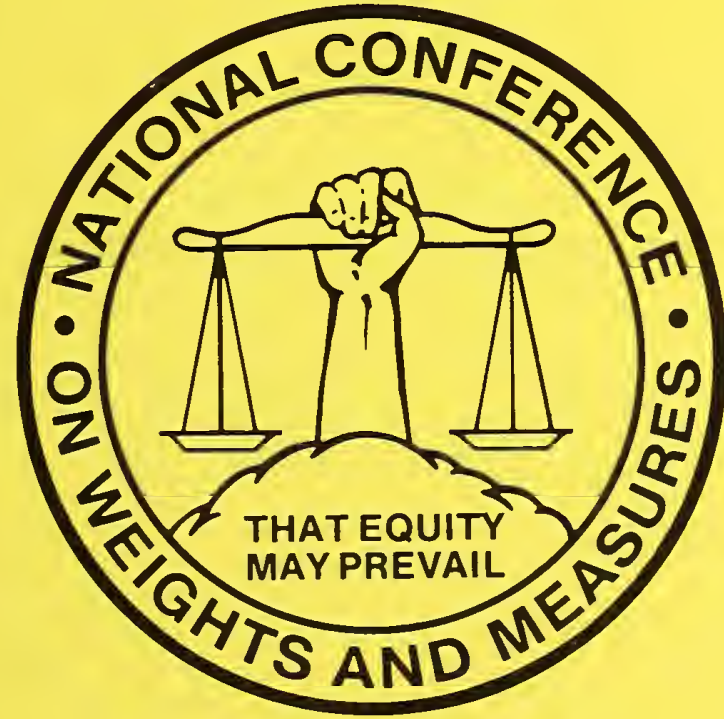

NCWM Publication 9

\author{
NBSIR 86-3374
}

$Q C$

100

.456

April 1986

$86-3374$ 



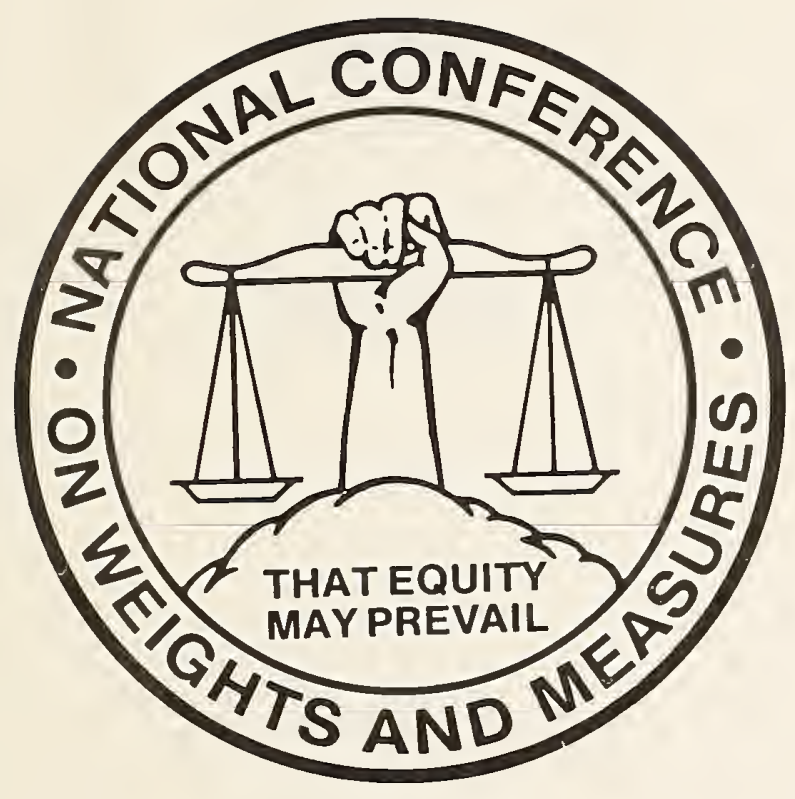

$\underline{1986}$

\section{DIRECTORY OF ASSOCIATE MEMBERS}

This Directory contains two types of listings of the Associate Members of the National Conference on Weights and Measures. Section I is a listing by the member's name. Section II is a listing by company name.

The names, addresses, and phone numbers huve been checked. However, due to reorganizations, retirements, and position changes implemented after this printing, some information may no longer be current. If a change, deletion, or addition is required, please send updated information to:

Ann P. Heffernan

Conference Coordinator

National Conference on Weights and Measures

P. O. Box 3137

Gaithersburg, MD 20878

Membership in the National Conference on Weights and Measures is $\$ 35.00$ per year. For the convenience of anyone wishing to become a member, an application is provided in the back of this publication. 


\section{TABLE OF CONTENTS}

\section{Section I}

$\underline{\text { Page }}$

Listing by Member

SECTION II

Listing by Company . . . . . . . . 41 

SECTION I

LISTING BY MEMBER 

MARKET I NG/SALES DEPT FISONS WESTERN CORPORATION $500-1212$ WEST BROADWAY VANCOUVER BC

$604873-3201 \times 207$

SALES MANAGER

G T MICHELLI CO INC

RIVER RIDGE, LA 70123

504 $885-9710$

WILBERT D ABELE

VICE PRESIDENT \& GENERAL MGR

TROEMNER HENRY INC

6825 GREENWAY AVE

PHILADELPHIA, PA 19143

$215724-0800$

LEROY ADAMS

NORWICH EATON PHARMACEUTICALS

$P O B O X 2468$

GREENVILLE SC 29602

$803277-7110 \times 298$

PEGGY H ADAMS

SECRETARY

PA ASSN WEIGHTS AND MEASURES

$P$ O BOX 340

BEDMINSTER. PA 18910

ROBERT $O$ ADERS

PRES IDENT

FOUD MARKET ING INST I TUTE

$1750 \mathrm{~K}$ STREET NA

WA SHING TON, DC 20006

$202452-8444$

FRANCISCO AGUIRRE

BI OCHEM IST

INST CENTRO AM INV TEC IND

AVENIDA LA REFORMA 4-47ZONA 10

GUATEMALA GUATEMALA 310631

HOWARD ALBOUM

TECHNICAL DIRECTOR

$J$ L PRESCOTT COMPANY

27 EIGHT ST POBOX 778

PASSAIC. NJ 07055

$201777-4200$

JAMES L ALCORN

SUPERVISORY LIVESTOCK SEW SPEC

$P E S-A M S$ US DEPT OF AGR ICULTURE

LIVESTOCK EXCH BLDG ROOM 208

DENVER. CO 80216

$303837-3312$

JOHN H ANDER SEN

PRESIDENT

NORTHWEST SVC STATION EQ CO

2520 NICULLET AVE

MINNEAP OLIS. MN 55404

$612827-5501$

ROBERT F ANDERSON

EXECUT I VE DIRECTOR

NATIONAL CHEESE INST

699 PRINCE ST/PO BOX 20047

ALEXANDRIA. VA 22320

312 263-2733

ROGER N ANDERSON

VICE PRESIDENT

COLUMBIA GRAIN INC

P O BOX 03370

PORTLAND, OR 97203

$503286-9681$
ROY ANDERSON

QUAL ITY ASSURANCE

OUAKER OATS

PO BOX 3040 FOODS DIVISION

SHIREMANSTOWN, PA 17011

$717737-8601 \times 282$

ROBERT J ANGELL

PRESIDENT

ANGELL EQUIPMENT COMPANY INC

PO BOX 667

PELHAM, AL 35124

$205663-6706$

THEODORE $R$ ANJOS

OEM SALES MANAGER

TRUCK-LITE CO INC

310 EAST ELMWOOD AVE

FALONER, NY 14733

$716665-6214 \times 261$

ROBERT W APKING

MFG SERVICES MANAGER

FRANKL IN INTERNATIONAL

2020 BRUCK ST

COLUMBUS, OH 43207

614 445-1404

KENNETH C APPELL

DIRECTOR-OUALITY ASSURANCE

COLGATE PALMOL IVE CO

300 PARK AVE

NEW YORK, NY 10022

$212310-2022$

CATHY A APPLE

FLOW SYSTEMS ENGINEFR

MICRO MOTION

7070 WINCHESTER CIRCLE

BOULDER, CO 80301

$303530-8530$

JOHN W AQUADRO

VICE PRESIDENT INDUSTRIAL OIV

HOWE RICHARDSUN

680 VAN HOUTEN AVE

CLIFTON, NJ 07015

$201471-3400 \times 243$

DONALO P ARNDT SEN

PRES IDENT

ACCURATE METERING SYSTEMS INC

$1731-33$ CARMEN DR

ELK GROVE. IL 60007

$312 \quad 640-0670$

GENE L ARNETT

MONSANTO COMPANY

WEST $10 \mathrm{TH}$ ST

ANNISTON, AL 36201

$205236-6381$

ROBERT C ARNOLD

EXECUTIVE VICE PRESIDENT

ARKANSAS OIL MARKETERS ASSOC

$P$ P BOX 229

LITTLE ROCK. AR 72203

$501374-6293$

MILTON H ARONSON

EDITOR PUBLISHER

MEASUREMENT AND CONTROL

2994 N LIBERTY AVE

PITTSBURGH. PA 15216

$412343-9666$

BERNARD ATHANE

DIRECTOR

BUREAU INT METROLOGIF LEGALE

II RUE TURGOT

PAR IS 75009 
JACK C AWBREY

QUALITY CONTROL SPECIALIST

SOUTHERN CO SERVICES FUEL DEPT 1036 2ND STREET N E

ALABASTER. AL 35007

$205877-7675$

DON AYERS

MANAGER QUAL ITY CONTROL

$J$ L PRE SCOTT CO

27 EIGHTH ST

PASSAIC. NJ 07055

$201777-4200$

GREG AYERS

OPERATIONS MANAGER

ALLAN U BEVIER CO

RD 3 BOX $85 B$

DELMAR, MD 21875

$301546-3293$

JAMES G AYERS

DELTA SCALE INC

4525 MULLENS FORD ROAD

CHARLOT TE NC 28226

$704527-8256$

GLEN E BABCOCK

SALES MANAGER

SIEINHOFFER SCALE CO INC

55645 CURRANT ROAD

MI SHAWAKA. IN 46545

$219259-5425$

DWANE L BAILEY

CHIEF OF WEIGHT CONTROL

BEECH AIRCRAFT CORPORAT ION

9709 E CENTRAL AVE

WICHITA. KS 67201

$316681-7875$

RICHARD E BAILEY

1815 MARVIN GRIFFIN/POB 5641 AUGUSTA. GA 30906

$404793-2190$

DONALD J BAIN

VICE PRESIDENT-COMPLIANCE

CONTINENTAL GRAIN CO

277 PARK AVE

NEW YORK NY 10017

MARIAN P BAIR

POWELL ALL-STEEL SCALES INC

RT 8 BOX 313

JASPER, AL 35501

$J$ D BAKER

ASST ENGINEER BALLAST SCALES

AT E SF RWY CD

4100 S KEDZIE AVENUE

CHICAGO IL 60632

EOWIN G BANKS

SUPERVISOR OF SCALES

SUUTHERN PACIFIC TRANSP CO

1 MARKET PLAZA ROOM 1007

SAN FRANCISCD. CA 94105

$415891-7828$

HARRY BARBEE

SERVICE MANAGER MILK COOLERS

PAUL MUELLER COMPANY

$P$ O BOX 828

SPRINGF IELD, MO 65801

$417831-3000 \times 205$
GEORGE J BARBER

SCALE TECHNITION

VAN DYKE SCALE CO

2825 RAYTOWN RD

KANSAS CITY. MO 64128

$816861-7191$

SALVATORE A BARBERA

PRESIDENT

WEIGHING \& AUTOMATION SYSTEMS

20 PASSAIC ST

GARF IELD, NJ 07026

$201777-5522$

TOM BARNET T

DIRECTOR RESEARCH \& DF VELOPMEN FRED STEIN LABORATOR IFS INC

121 NORTH FOUR TH ST

ATCHISON, KS 66002

$913367-3945$

R D BARTLETI

MGR REFRIG ENG

BAESON BRDS CO

$2100 \mathrm{~S}$ YORK RD

OAK BROOK, IL 60521

$312654-1600 \times 127$

HARRY G BAUGHN

PRES

PACIFIC SCALE CO

3236 SE 50TH

PORTLAND, OK 97206

$503777-3978$

HOWARD E BAUMAN

$\checkmark P$ SCIENCE \& REGULATORY AFFAIR PILLSBURY CUMPANY

311 SECOND ST SE

MINNEAPDLIS. MN 55414

$612330-4076$

JOHN S BAUMANN

VICE PRESIDENI

NEW BRUNSWICK INTERNATL INC

5 GREFK LANE

EDISON. NJ OB817

$201287-2288$

JAMES G BECKER

PRES IDENT

ELECTROMEIRICS CO

6433 HAVELOCK AVE

LINCOLN. NE 68507

$402467-3541$

KEVIN C BEGLEY

CUSTOMER SERVICE

GENERAL ELECTKO DYNAMICS CORP

P O BOX 15008

ARL INGTON, TX 76015

IRVING BELI

SR EXECUIIVE STAFF REPR

COCA-COLA COMPANY

PO URAWER 1734

A IL AN I A. GA 30301

$404676-2623$

JAMES BELL

SALES AND INFORMATION

MIDWESTERN SCALE CO

1 PECAN PLALA

NEVADA. MU 64772

$417667-6333$

JAMES E BELL

PRESIDEN I

EOUIPMENT CONTROLS CO

4555 BFRKELEY LAKF RN

NORCROSS, GA 3007

$404448-4531 \times 008$ 
F MICHAEL BELUE

MECHANICAL ENGINEERING DEPT

SOUTHWEST PUHP COMPANY

PQ DRAWER 280

BONHAM, TX 75418

$214583-3134 \times 47$

DOWARD $T$ BENEFIELD

QUALI TY CONTROL MANAGER HUMKO PRODUCTS KRAFT CORP

$P$ O BOX 398

MEMPHIS, TN 38101

RICHARD BENTLEY

MANAGER SCALE DEPT

WEBB CORPORAT ION

$P$ DOX 549

WEBB CITY. MO 64870

$417673-4646$

GERALO J BERGER

SALES MANAGEER

TECNETICS INDUSTRIES

2180 OLD HIGHWAY 8

$612780-4880$

EDWARO L BERGMANN

MGR QUALITY ASSURANCE PET FOOD

RALSTON PURINA CO

900 CHECKERBOARD SQUARE

ST LOUIS, MO 63188

$314982-3483$

RONGNER BERGMARK

PRESIDENT

FRANKLEN EQUIPMENT INC

6643 G3RD ST

CHICAGO. IL 60638

$312586-7881$

CHRISTOPHER P BERNE

PRESIDENT

BERNE SCALE COMPANY

2206 EDGEWOOD AVE S

ST LOUIS: MN 55426

$612544-2422$

ROY S BERRYHILL

VEHICLE MAINTENANCE

THURSTON AVIATION INC.

3840 A I RPORT DRIVE

CHARLOTTE: NC 28219

$704394-4331$

ROBERT H BET TINUEER

PRESIDENT

AGRI-ELECTRONICS SYSTEMS ING 12601 ECKEL ROAD

PERRYSBURG: OH 43551

$419537-6776$

FR.EDERICK W BETZ

$S$ WETZ CO INC

2239 KIRK AVE

BALTIMORE, MD 21218

$301366-1030$

ROBERT BIXLER

SCHEK ING CORPORATION

1011 MORRIS ST

UNION, NJ OTO83

$201931-2000$

HERMAN BLITZSTEIN

PRESIDENT

OMEGA BALANCE SERVICE INC

$P$ O BOX 325

MATAWAN, NJ 07747

$201946-2121$
M JEFFREY BLOOM

QUALITY CONTROL MANAGER

AGR I-MARK INC

$P$ O BOX 5800

LANRENCE, MA 01842

$617689-4442 \times 222$

BARRIE L BLOSER

CHIEF ENGINEER

BROOKS INSTRUMENT DIVISION

HWY 301 NORTH BOX 450

STATESBORO: GA 30458

$912764-5471 \times 284$

JUEL BLUMENTHAL

MICRO MOTION INC

7070 WINCHESTER CIRCLE

BOULDER, CO 80301

$303530-8400$

JAMES BOGGS

MANAGER OF ENERGY $\&$ UTILITIES

BURG WARNER CHEMICALS

PO BOX 658

OTTAHA. IL 61350

$815434-7000 \times 206$

ROGER B BOGNAR

MANAGER TISSUE DIVISION

AMER ICAN PAPER INSTITUTE

260 MADI SON AVE

NEW YORK. NY 10016

$212340-0618$

DON E BOLIN

MANAGER OF QUALITY CONTROL

LIBERTY GLASS CO

PO BOX 520

SAPULPA, OK 74006

G18 224-1440 $\times 244$

LOUIS P BONAPACE

PRESI DENT

INEW BRUNSWICK INTERNATL INC

5 GREEK LANE

EDISON, NJ 08817

$201287-2288$

MARY J BOND

LIBRAR I AN

ADOLPH COORS COMPANY

MAIL BC520

GOLDEN, CU 80401

$303277-3506$

EDWIN E BOSHINSKI

DIR ADVANCED DEVEL OPMENT

HOBART CORPORATION

WORLD HEADQUARTERS

TROY. OH 45374

$513332-2637$

JACQUES C BOUBILLE

PRESI DEN T

METRO EOUIPMENT CORPORATION

1235 REAMWOOD AVE

SUNNYVALE, CA 94086

$408734-5400$

JUSEPH $W B O W$

EXECUIIVE VICE PRESIDFNT

SINGLE SERVICE INSTITUTF

1025 CONNEC IICUT AVF NW

WASHINGTON. DC 20036

$202347-0020$

WILLIAM T BOWLES

DIRECTOR PREP ENGINFER ING

PITISBRG-MIDNAY COAL MINING CO

1720 S BELLAIRE ST

DENVER. CO 80222

$303158-1700 \times 386$ 
ANDREW G BOWMAN

OWNER

ANDREW BOWMAN CO INC

B5T SO HOFFMAN LANE

CENTRAL ISLIP, NY 11722

$516234-6669$

DARRY L BOYD

ELECTRONIC ENGINEER ING TECH DE TROIT EDISON COMPANY

GIOO W WARREN

DETROIT, MI 48210

$313897-1191$

CHESTER D BRAOLEY

VICE PRESIDENT

A H EMERY COMPANY

RURAL RT 2 BOX 179

HEAIHSVILLE, VA 22473

$304580-4305 \times 37$

JACK B BRADLEY

PRESIDENT

INLAND NORTH WEST SCALE CO
PO BOX 11335 5602 E DESMET

SPOKANE WA 99211

$509535-4295 \times 323$

KEITH W BRADLEY

AS ST CHIEF ENGR-PLANNING

DENVER \& RIO GRANDE WESTERN RR

1515 ARAPAHOE ST PO BDX 54 B2

DENVER, CO BO 217

$303629-5533 \times 2433$

RICHARD S BRADLEY

PRESIDENT

WE I GH-TRONIX INC

1000 ARMSTRONG DR

FAIRMONT, MN 56031

$507238-4461 \times 200$

JOHN B BRADY

PRE SIDENT

JBBRAOY INCORPORATED

BII N AL VORD ST

SYRACUSE, NY $1320 B$

$315422-9271$

WILLIAM D BRASHER

QUALITY CONTRUL SPECIALIST

SOUTHERN COMPANY SERVICES

$P$ O BOX 2625

BIRMINGHAM, AL 35202

$205877-7653$

EDWARD BRATLE

RETAIL SYSTEMS $D \& P$ DIVISION

NCR COR PORATIION

WORLD HEADQUARTEKS 4 TH FLOOR

DAYTON, OH 45479

WILLIAM H BRAUN

PACKAGING SECTION HEAD

PROCTER \& GAMBLE

6IOO CENTER HILL RO

$513659-5233$

GARY BRENNGLASS

PRESIDENT

PACKAGING CORP OF AMERICA

4633 DU WNEY ROAD

LUS ANGELES, CA $9005 B$

$213589-8181$

WILLIAM H BREWER

MGR RESEARCH E DEVELOPMENT

OARIGOLD INC

P O BOX CI 9099

SEATTLE, WA 98119

$206284-7220$
JOHN F BREWER JR

MANAGER CONST E MAINT

FINA OIL E CHEMICAL COMPANY

$P O B O X 2159$

DALLAS, TX 75221

$214750-2642$

CHARLES H BROKAW

DIRECTOR OF PRODUCT INTEGNITY EDWARDS BAKING COMPANY

I LEMON LANE

ATLANTA, GA 30307

$404377-0511$

BOB BRONSON

MANAGER OF $R \& D$

NAT IONAL CONTROLS INC

4500 JOHN YOUNG PARKWAY

ORLANDO. FL $32 \mathrm{BO} 04$

$305298-0506 \times 230$

DOUGLAS G BROOKS

PRESI DENI

LODEC INC

PO DRAWER D

LYNNWOOD, WA 980461300

$206775-6471$

DONALD A BROWN

PROJECT ENGINEER

CAL IFORN IA ALMOND GROWERS EXCH PO BOX $176 B$

SACRAMENTO. CA 95808

$916446-B 386$

LEE BROWN

CONTINENTAL FOOD PRODUCTS CO

RE-MI FOUD PRODUCTS

1201 TONNE RUAD

ELK GROVE, IL 60007

$312640-0400$

MACK BROWN

OPERATIONS MANAGER

CONTINENTAL GRAIN CO

5100 OAKLAND AVE

ST LOUIS. MO 63110

$314531-8300$

CHARLES W BROWN SR

VICE PRESIDENI

ANN ISION PUMP SHOP INC

$2 B O 0$ HIWAY $431 \mathrm{~N}$ PO BRX 1198

ANN I STON, AL 36201

$205820-2980$

ROBERT BRUCE

ACT ING CHIEF WIS AND MEASURES

CONSUMER \& CORPURATE AFFAIRS

OITAWA ONT

$613996-3035$

NORMAN R BRUCKER

REGIONAL SCALE INSPFCIOR

BURLINGION NOR IHERN RAILROAD

176 EAST 5 TH ST BOX 64960

ST PAUL. MN 55164

$612298-2605$

ROBERT T BRUMBAUGH

PRES I DENT

SYSTEMS ASSOCIATES INCORPORAIF

205 PEIERSON RD

LIBERTYVILLE. IL 60048

$312367-0650$

PRESTON B BRYANT

ARI DIRECTOR

VENTURE PACKAGING

PO BOX 7149

CHARLETTE, NC 28217

$704588-0220 \times 9$ 
DAUN A BRYDON

DIRECTOR PUBLIC AFFAIRS

ICE CREAH HNFRS ASSN

$88816 \mathrm{TH} S T \mathrm{NW}$

HASHING TON DC 20006

$202296-4250$

MILLIAH H BUCHANAN

REGULATORY AFFAIRS

SUNNYSIDE CORPORATION

225 CARPENTER AVENUE

WHEELING $I L$ IL 60090

$312541-5700 \times 0022$

RONALD L BUCK

PRE SIDENT

SCALE PEOPLE INC

10101 B ACON DR

BELTSVILLE: MD 20705

$301949-6994 \times 215$

R N BUMGARDNER

E I DUPONT DE NEMOURS \& CO 901 D DUPONT AVE

BELLE, HV 25015

$304949-4313$

JOHN H BUNGENER

VICE PRESIDENT OPERATIONS

PELOUZE SCALE CO

$P$ O BOX 1058

EVANSTON: 11260204

T A BURCH

ANDERSON CLAYTON FOODS

$3333 N$ CENTRAL EXPWY

RICHARD SON, TX 75080

$214231-6121$

KEITH A BURDICK

OUALITY AS SURANCE MANAGER KOLMAR LABORATORIES INC

P BOX 1986

DENTON TX 76201

GERALD R BURGER

COAL SUPPLY COORDINATOR

CONSUMERS POHER CO

1945 PARNALL RD

JACKSON, MI $\$ 9201$

$517788-0114$

PETER A BURKE

LABORATORY MANAGER

KIWI BRANDS INC

ROUTE 662 NORTH

DOUGLASSVILLE, PA 19518

$215385-3041$

E E BURMAN

NATIONAL ACCT MGR AGRI IND

CARDINAL SCALE HFG CO

$P$ O BOX 24-061

APPLE VALLEY. MN 55124

$612432-2100$

MAHLON A BURNETTE III

PUBLIC AFFAIRS CONSULTANT

GROCERY MANUFACTURERS OF AMER

631 HALKER ROAD

GREAT FALLS, VA 22066

$703759-5984$

WILLIAM H BUTTERBAUGH

ASST VICE PRES TECHNIC SERV

NAT IONAL LP-GAS ASSN

1301 WEST 22ND ST

OAK BROOK IL 60521

$312986-4800$
KEITH H CABLE

NORTHHEST CALIBRATION SYSTEMS

510 S LUCILE ST

SEATTLE, WA 98108

SHELDON H CADY

EXECUTIVE VICE PRESIDENT

MINERAL INSULATION MFGRS ASSOC

382 SPR INGF IELD AVE

SUMMIT: NJ 07901

$201277-1550$

JACK R CALDICOTT

PRE SIDENT

AUTOMATIC MEASUREMENT TECHNOL

1000 RAND ROAD BLDG 111

HAUCONDA, IL 60084

$312526-0808$

RICHARD CALKINS

HETROLOGIST

RICE LAKE BEARING INC

RICE LAKE, HI 54868

$715234-9171$

ALAN CAMERON

MANAGER OUALITY ASSURANCE

OUALITY METER INC

507 MISSOURI AVENUE

HEST PLAINS: MO 65775

$201687-4000$

MICHAEL P CAMPBELL

PRODUCT MANAGER

OHAUS SCALE CO

29 HANOVER ROAD

FLORHAM PARK. NJ 07932

$201377-9000$

HUGO M CANCIO

ENG INEER ING MANAGER

SYSTEMS INSTALL SPECS CO INC

PO BOX 10310 CAPARRA HGHTS STA

SAN JUAN PR 00922

809 783-1057

JEFFREY D CANFIELD

PRE S IDEN I

ACME SCALE \& SUPPLY CO

5401 BUTLER ST

PITTSBURGH,PA 15201

$412782-1808 \times 1$

A M CANNAVA

14911 ROLLING RDG/PO BOX 2437

CHINO, CA 91708

JACKIE CAPLINGER

OUALITY ASSURANCE MANAGER

WYANDOT INC

135 HYANDOT AVE

MAR I ON, OH 43302

$614383-4031$

ROBERT S CARLES

ATTORNEY

$\angle A N C E$ INC

PO BOX 32368

CHARLOTIE NC 28232

$704554-1421$

GEORGE E CARLETON

MGR ADMIN \& SPECIAL SERVICES

PROCTER \& GAMBLE CO

ONE PROCTER \& GAMBIE

CINCINNATI: OH 45202

PLAZA

$513562-2721$ 
JOHN L CARLTON

PRESIDENT

CAHCAL COMPANY

4000 AIRPORT WAY SOUTH

SEATTLE HA 98108

$206682-5416$

RALPH」CARNEY

ANALYTICAL BALANCE SERVICE

105 BRADLEY CIRCLE

DURHAM. NC 27713

$919544-7003$

LAVERN H CARROLL

CHIEF SCALE INSPECTOR

ILLINOIS CENTRAL GULF RAILROAD

600 GILMORE ST

CENTRAL IA: IL 62801

$618533-3319$

ROBERT H CARTER

IRHIN HEASURING TOOL CO

P 0 BOX 471

PATCHOGUE, NY 11772

$516289-0500$

LLOYD CARVER

METROLOGY SERVICES CORP

P O BOX 7029

HUNTSVILLE, AL 35805

$205534=0074$

MAX C CASANOVA

MANAGER FIELD SERVICE

RAMSEY ENGINEERING COMPANY

$1853 W$ COUNTY RD $C$

SAINT PAUL MN 55113

$612633-5150 \times 264$

DAVID CERVANTES

PROJECT ENG INEER

ELECTROSCALE CORP

P O BOX 1786

SANTA ROSA CA 95402

WILBERT G CHAMBERS

FISCA OIL CO INC

$P$ O BOX 3363

KANSAS CITY, KS 66103

NORMAN G CHAMPINE

LAB ORATORY MANAGER

OMYA INC

61 MAIN STREET

PROCTOR VT 05765

$802459-3311 \times 219$

JEOFFRE H CHAN

ASSISTANT MANAGER

OTEX FACTOR

2040 TAFT AVE

PASAY CITY-MANILA

LEONARD S CHANDLER

OUALITY CONTROL MANAGER

LUDENS INC

200 NOR TH 8 TH ST

READING, PA 19603

$215376-2981$

STEPHEN L CHARLTON

AARROW EQUIPMENT COMPANY INC P O BOX $665 / H W Y 52$ ARMSTRONG PELHAM, AL 35124

$205663-5858$
PAUL CHASE

VICE PRESIDENT OF RES \& DEVEL

RAMSEY ENG INEER ING COMPANY

1853 W COUNTRY ROAD C

ST PAUL: MN 55113

$612633-5150$

JOHN CHESLEY

PRESIDENT

DERLEIN SCALES \& MFG INC

2425-0 S STOUGHTON RD

MADISON, HI 53716

$608222-0606$

CHOU CHI-HSIANG

DIRECTOR GENERAL

NATIONAL BUREAU OF STANDARDS

$4 T H$ FL 102 KWANG-FU S ROAD

TAIPEI TAIWAN

JOHN J CHINNI

PRESIDENT GEN MGR

TEXAS SCALES AND MATERIAL HDLG RT 3 BOX $183 \mathrm{D}$

CIBOLO. TX 78108

$817848-4491$

R KEVIN CHUMNEY

MGR GOVT REGULATIONS

CAMPBELL SOUP CO

CAMPBELL PLACE

DWAYNE E CIRCLE

CHIEF TRAVELING AGENT

WESTERN WEIGHING E INSP BUREAU

3435 BROADHAY SUITE 201

KANSAS CITY. MO 64111

$816753-2101 \times 17$

STANLEY L CISIEWSKI

MANAGER CUSTOMER SERVICE

PEABODY COAL COMPANY

$80 \times 14495$

ST LOUIS. MO 63178

$618398-7950 \times 222$

GARY CLARK

MANAGER REGULATORY AFFAIRS

12200 DENTON DR

DALLAS. TX 75234

$214243-2321$

WARREN S CLARK JR

EXECUTIVE DIRECTOR

AMERICAN DRY MILK INSTITUTE

$130 \mathrm{~N}$ FRANKLIN ST

CHICAGO. IL 60606

$312782-4888$

LAVAR CLEGG

DIRECTOR OF ENGINEERING

TRANSDUCERS INC

CERRIJOS CA 90701

$714739-1991$

JUDY CL INE

ASST TO TECHNICAL DIRECTOR

CARPET AND RUG INSTITUTE

PO $80 \times 2048$

DAL TON. GA 30720

$404278-3176$

DANIEL J COCKRELL

PRESIDENT

WEIGHING \& CONTROL SYSTEMS INC

$P$ O BOX 1483

BRANDON, FL 33511

$813681-5733$ 
SAMUEL O COEN

SENIOR RESIDENT ENGINEER

PO BOX 1673

AL TOONA:PA 16603

$814695-4476 \times 78$

DHIGHT A COHAGAN

DIR REGULATORY SERVICES

SHERWIN-WILLIAMS COMPANY

101 PRO SPECT AVE NH

CLEVELAND, OH 44115

$216 \quad 566-2918$

HENDRIK COLIJN

CONSULT ING ENGINEER

423 FRANKLIN HTS DR

MONROEVILLE, PA 15146

$412372-8130$

JIM H CONNER

EXECUTIVE VICE PRESIDENT

AMERICAN YARN SP INNERS ASSOC

PO $80 X 99$

GASTONIA. NC 28053

$704867-7201$

D P CONYERS

QUALITY $E$ SPECIFICATIONS MGR OHENS ILLINOIS INC

1 SEA GATE

TOLEDO: OH 43666

$419247-0489$

CHARLES $F$ COOK

OSCAR MAYER \& CO

OSCAR MAYER FOODS CORP

$P$ O $80 \times 7188$

MADISON, HI 53707

$608344-6819$

DON P COOK

OWNER

FLORIDA INDUSTRIAL SCALE CO

1540 NORTH ST

LONGWOOD: FL 32750

A G COOPER

MANAGING DIRECTOR

SALTER INDUSTRIAL MEASUREMENT

GEORGE STREET

WEST BR OMWICH

THOMAS G CORDELL

EXECUT I VE DIRECTOR

MICHIGAN PETROLEUM ASSOCIATION

1200 MICHIGAN NATIONAL TOWER

LANSING MI 48933

$517487-9139$

RO8ERT 8 CORNEY

MILLER 8REWING CO

3939 WEST HIGHLAND 8LVD

MILHAUKEE WI 53208

$414931-4222$

KEN COSBY

MANAGER COMPUTER CONTROLS DIV

CMI CORPORATION

P O BOX 1985

OKLAHOMA CITY. OK 73101

RICHARD C COVINGTON

MANAGER QUALITY CONTROL

AMERICAN FINE FOODS INC

$P$ O $80 X 460$

PAYETTE. ID 83661

$208642-9061$
ROBERT N COX

PRES I DENT

COX FIXTURE \& SUPPLY INC

3412 JACKSONVILLE HWY

NORTH LITTLE, AR 72117

$501945-6011$

VERNON L CRAIG

CHIEF INSPECTOR

MO8AY CHEMICAL CORP

PO BOX 4913 HAWTHORN ROAD

KANSAS CITY. MO 64120

$816242-2000 \times 2416$

PHIL CRAIN

DIRECTOR QUALITY ASSURANCE

CALRECO

8015 VAN NUYS BLVD

VAN NUYS, CA 91412

$818787-7820 \times 225$

JOHN T CRAMBES

PLANT MANAGER

$U$ S TOBACCO CO

11601 COPENHAGEN COURT

FRANKLIN PARK IL 60131

$312595-8200$

KEITH A CRANDALL

DIRECTOR OF SALES AND SERVICE CRANDALL FILLING MACHINERY INC P0 $80 \times 706$

BUFFALO, NY 14217

$716885-2228$

STANLEY CRANDON

SCIENCE APPLICATIONS INC

SCIENCE APPLICATIONS INTL CORP POMONA. CA 91766

$714623-6711$

ROGER CRAHFORD

OWNER

LA8 8 ALANCE \& MICROSCOPE SVC

203 SCENIC DR

OAK RIDGE: TN 37830

$615483-9652$

RONALD J CRAHFORD

CHIEF INSPECTOR W \& M

CONSUMER AFFAIRS BUREAU

BRISBANE 252

DONALD E CRIPE

CORPORATE DIR QUALITY SYSTEMS

SCHREI8ER FOODS INCORPORATED

P $080 \times 19010$

GREEN 8AY. WI 54307

$414437-7601 \times 581$

WILLIAM J CRISAFI

DIRECTOR OF MANUF ACTUR ING

HEBSTER INDUSTRIES INC

58 PULASKI ST

PEABODY. MA 01960

$617532-2000$

CLARENCE N CROCKER

MGR TECHNICAL SERVICES

AMAX COAL COMPANY

105 S MERIDIAN ST P O BOX 967

INDI ANAP OL IS. IN 46206

$317266-2824$

HENRY E CROS 8Y

ASST DIR CONSTRUCTION \& MAINT PUBLIX SUPER MARKETS INC

$P$ O BOX 407

LAKELAND FL 33802

$813686-1188 \times 281$ 
RONALD E CROW

OA MANAGER

HILLS PET PRODUCTS

$B 0 X 148$

TOPEKA. KS 66603

$913354-8340 \times 38$

JAMES CULLEN

SCALE TECHNICIAN

KITCHENS OF SARA LEE

500 WAUKEGAN RO

DEERFIELD: IL 60015

$312945-2525 \times 2282$

ALAN $P$ CULVER

PERDUE INC

$P$ O BOX 1537

SALISBURY, MD 21801

RICHARD J CUNNINGHAM

ENGR CONST \& MAINT

ATLANTIC RICHFIELO COMPANY

515 SOUTH FLOWER ROOM 1809

LOS ANGELES:CA 90071

CECIL J DAMERY

PRESIDENT

DECATUR DANVILLE SCALE CO INC

120 S WALL ST PO

MACON: IL 62544

$217764-3341$

A R DANIELS

DIRECTOR INOUSTRY STOS \& REL

NCR CORPORATION

1700 S PATTERSON BLVD WHO

DAYTON, OH 45479

$513445-1310$

CARL E DARIGO

DIRECTOR CORPORATE QUAL ASSUR

ANHEUSER-BUSCH INC

ONE BUSCH PLACE

ST LOUIS. MO 63118

$314577-3966$

DEBRA DATTILO

CHEMIST-REGULATORY-AFFAIRS

BENJAMIN MOORE \& CO

134 LISTER AVE

NEWARK: NJ 07105

$201344-1200 \times 42$

KENT E DAVENPORT

ENGINEER FUELS

COLORADO-UTE ELECTRIC ASSN INC

$P$ BOX 1149

MONTRDSE. CO 81402

303 249-4501

JAHES W DAVIDSON

ASS ISTANT CHIEF ENG INEER

BURLINGTON NORTHERN RA ILROAD

$P$ O BOX 29136

OVERLAND PARK, KS 66201

$913661-4238$

JEFFREY B DAVIES

MARKETING DIRECTOR

CMI IDEARBORN

820 LAFAYETTE RD BLDG 1 \$203

HAMPTON, NH 03842

$603772-9791$

BILLYE DAVIS

DAVIS REFRIGERATION SVC INC

1207 SO CHURCH ST

ROCKY MOUNT, NC 27801
GERALD WAVIS

SERVICE MANAGER

HOBART CORPORATION

10631 SUAMIT ST

LENEXA: KS 66215

$913469-9600$

RICHARD L DAVIS

ADMINISTRATOR REGULATORY COMPL

JAMES RI VER CORPORATION

1915 MARATHON AVE

NEENAH, WI 54956

$414729-8174$

PETER DE KORTE

PRESIDENT

EMPRO PRODUCTS COMPANY INC

357 MCLEAN BLVO

PATERSON. NJ 07513

$201279-1010$

TOM DE RYKE

MGR ENGINEERING AND PROD DEVEL BERKEL INC

1 BERKEL DRIVE

LAPORTE. IN 46350

DENNIS T DEDOMENICO

SECRETARY-TREA SURER

GOLDEN GRAIN MACARONI CO

$1111139 \mathrm{TH}$ AVENUE

SAN LEANDRO. CA 94578

$415357-8400 \times 324$

VINCENT J DEL GIUDICE CONSULTANT

DEL GIODICE ASSOCIATES

6620 N HAUKESHA AVENUE

CHICAGO. IL 60646

$312763-1183 \times 3075$

VICTOR DEL ROSSO

PRESIDENT

HI-SPEED CHECKHEIGHER CO INC

605 HEST STATE ST

ITHACA. NY 14850

$607273-5704$

NANCY J OEMARCO

EXECUTIVE DIRECTOR

INDEPENDENT LUBRICANT MFR ASSM

1055 JHOMAS JEFFER SON NH $\$ 02$

WASHINGTON, OC 20007

$202337-3470$

C J DEMPSTER

GRAIN RE SEARCH LABORATORY

CANADIAN GRAIN COMMISSION

1404-303 MAIN ST

WINNIPEG R $3 C$

JOHN P DENHAM

SYSTEMS ENGINEER

TIME OIL CO

2737 COMMODORE HAY

SEATTLE. HA 98199

$206285-2400 \times 61$

CHARLES J OENNY

MGR CUSTOMER \& TECHNICAL SERV

HILLIAM M WILSONS SONS INC

$8 \&$ VALLEY FORGE RDS

LANSDALE. PA 19446

$215855-4631 \times 37$

LEE C DESKIN

PRES I DEN

DESKIN SCALE CO INC

437 W MC GEE ST

SPRINGF IELD. MO 65804

417883-0055 
TOM DETERMAN

DETERMAN HELDING \& TANK

$124172 \mathrm{ND} A Y$ NE

MINNEAPOLIS, MN 55432

$612571-8110$

JOSEPH F DEVITT

SERVICE MANAGER

US HWY $\angle 22$

PLAINFI ELD. NJ 07061

$201757-1600 \times 2723$

LOUIS C DIANI

SER YICE DEPARTMENT

MERRICK SCALE MFG COMPANY

MERRICK DRIVE

ROSELAND. NJ 07068

$201226-0656$

RALPH T DICKSON

SCALE TECHNICIAN

SELF-EMPLDYED

RT 3 BOX $246-E$

CHINA GROVE, NC 28023

BEN DILLON

PRESIDENT

MASSTRON SCALE INC

6600 HUNTLEY ROAD

COLUMBUS, OH 43229

$614 \quad 436-3292$

EXECUTIVE DIRECTOR

NATIONAL STANDARDS COUNCIL

$P$ O BOX 5286 BOROKO

PAPUA NEH

ER IC D DIXON

PRES \& GEN MGR

STERL ING SCALE CO INC

20950 BDENING DRIVE

$313358-0590 \times 25$

FRANK A DOLEZAL

PRESIDENT

UNI TED STANDARDS LAB INC

CAL IBRATION SVC -4250 NICOLLET

MINNEAPOLIS, MN 55409

$612823-4438$

W GARY DOLL

$\checkmark$ ICE PRESIDENT-MARKETING

GENERAL ELECTRONIC SYSTEMS INC 914 SE 14 TH PLACE

CAPE CORAL FL 33904

$813574-2313$

GEQRGE S DOMINGUEZ

EXECUTI VE DIRECTOR

OXYGENATED FUELS ASSN

1330 CONNECTICUT AVE 300

HA SHING TON. DC 200361702

$202659-0060$

TED DONALOSON

PRESIDENT

CENTRAL SCALE INC

4915 E $16 \mathrm{TH}$ ST

INDIANAPOLIS. IN 46201

317 356-8005

W'L DOOLEY

GENERAL MANAGER

H $\&$ S ENTERPRISES

P O BOX 1050

FARMINGTON. NM 87499
MICHAEL R DOTY

QUALITY CONTROL SUPERVISOR

CHURCH AND DWIGHT CO INC

BOX 123

GREEN RIVER, WY 82935

$307875-2233 \times 124$

GLENN J DOUGLAS

DIRECTOR OF MARKETING

WORLD WIDE WEIGHING

$11863124 \mathrm{TH}$ AVE NE

KIRKLAND. HA 98034

$206821-8020$

JOHN M DUMAIS

EXECUTIVE DIRECTOR

NEW HAMPSHIRE RETAIL GROC ASSN

P O BOX 868

MANCHESTER NH 03105

$603669-9333$

CHARLES J DUNAWAY

STAFF ENGINEER - TECH MARKETING

CONSOL IDATION COAL CO

1800 WASHINGTON ROAD

PITTSBURG. PA 15241

$412831-4388$

A D DUNCAN

PRESIDENT

METERS INC

HWY 205 \& VICTORIA ROAD

WOODSTOCK, GA 30188

$404926-2917$

RICHARD D DUNCAN

SER VICE MGR

TECHSCIENCE INTERNATIONAL INC 3020 NO HESPER IAN WAY

SANTA ANA. CA 92706

714 558-6911

DONALD W DYCUS

OWNER

ARKANSAS SCALES INC

ROUTE 8 BOX 156

JONESBORO. AR 72401

501 935-0505

DAVID N EBBERT

MANAGER

STABRO LABORATORIES INC

25 KENS INGTON AVE

SALT LAKE, UT B4115

$801487-1344$

KAREN S EBLE

ARCO CHEMICAL COMPANY

1500 MARKET ST CS-3301G

PHILADELPHIA? PA 19101

$215-557-3635$

HAROLD H ECKE

PRE SIDENT

COMPUWE I GH CORP

PO BOX 4517 YALESVILLE STATION WALLINGFORD. CT 06492

$203284-9184$

BILL EDMAN

OWNER

BILLS PUMP \& REPAIR

RR2 BOX 259-H

LAWRENCE KS 66046

$913841-1758$

VICKI EDWARDS

EDWARDS EQUIPMENT CO INC

261-C W FLEMING DR

MORGANTON. NC 28655

704 433-0650 
HANS EIGENMANN

TECHNICAL SALES

$\mathrm{BOX} 71$

HIGHTSTOWN. NJ 08520

$609448-3000 \times 254$

JOHN J ELENGO JR

VICE PRESIDENT

REVERE CORPORATION DF AMERICA

$845 N$ COLONY RD P O BOX 56

WALLINGFORD. CT 06492

$203269-7701$

JOHN S ELLIOTT JR

GEN MGR COMPLIANCE AUDITS

HEINZ USA DIV OF H J HEINZ CO 1062 PROGRESS ST

PITTSBURGH PA 15212

$412237-5862$

CHARLIE ELLIS

OWNER

CEE SCALES \& EQUIPMENT CO

PO $80 X 700$ (104 PT GIBSON RD)

RAYMOND. MS 39154

$601857-5143$

\section{ROGER H ELIS}

MANAGER STANDARDS \& CAL LAB

EGEG IDAHO INC

PO BOX 1625

IDAHO FALLS, ID 83415

$208526-2656$

RUTHERFORD L ELLIS JR

VICE PRESIDENT-ELECTRONICS

UNI VERSEL EPSCO INC

1494 ELLSWORTH IND DR $8 \times 93544$ ATLANTA: GA 30318

$40435 \mathrm{I}-2740$

DOUGLAS R ENGEBRETSON

DIR QUALITY ASSURANCE \& TECHN LAND O LAKES INC

P D BOX 116

MINNEAPOLI S. MN 55440

$612331-6330 \times 390$

A W E ENGLE

INT SOC OF WEIGHING \& MEAS

2506 GROSS PDINT RD

$312475-1008$

DAVID C ENGL I SH

PRE SIDENT

MEA SUREMENT SYSTEMS INTERNTL

12622 INTERURBAN AVE S

SEATILE, WA 98168

$206767-7433$

LINDSEY $L$ EPPERSON

GEN MGR

INDUSTRIAL SCALE SERVICE

RT 2 BOX 355

MARSHALL. TX 75670

$214935-3027$

ALBERT ERK

OWNER

ERK SCALE CO INC

RT 4 BOX 262-B
FRANKLINTON, LA 70438

$504839-5660$

R C ERNST

WARNER LAMBERT PHARMACEUT ICAL

201 TABOR RD

MORRIS PLAINS. NJ 07950
ALFRED C EVANS

VICE PRESIDENI

VEE DER-RDOT CO

70 SARGEANT ST

HAR TFORD CT 06102

$203527-7201$

TIMOTHY $R$ EVANS

ATTORNEY

HOLBROOK JONSON BRESSLER ET AL

315 S MONUMENT AVE

HAMILTON, OH 45011

513 B68-7600

HERVE H FAFARD

PRESI DEN T

CONRAD FAFARD INC

PO $80 \times 3033$

SPRINGFIELD, MA 01101

$413786-4343$

JOHN J FAHY

MARKETING DIRECTOR

TRANSDUCERS INCORPORATED

I 4030 BOLSA LN

CERRITOS CA 9070

$714739-1991 \times 221$

FRED J FANDERS

OAMGR

CPC BEST FOODS

1120 COMMERCE AVE

UNION. NJ 07083

$201688-9000 \times 283$

BARBARA J FAUL KNER

VICE PRESI DENT

PETROLEUM MARKETERS ASSN OF AM

1120 VERMONT AVE SUITE 1130

WASHINGTON, DC 20005

$202331-1198$

JORGE L FAZ

DIRECTOR GOVT \& INDUSTRY AFFRS

ARCO CHEHICAL COMPANY

1500 MARKET ST

PHILADELPHIA. PA 19101

GLENN R FEILNER

ELEVATOR MANAGER

AGR I EXPORT

1606 CLINTON DR

GALENA PARK, IX 77547

$713672-2536 \times 52$

SY FE INL AND

PITNEY BOWES INC

MAIN AVE

NORWALK. CT 06851

$203853-7113$

RUTHERFORD H FENN

DIRECTOR CORPORATE STANDARDS

PITNEY BOWES

WAL TER WHEELER DRI VE

STAMFORD. CT 06926

$203853-0727$

ANTHONY M FESTA

GROUP LEADER

NOR TON CO

1 NEW BOND ST

WORCESTER, MA 01606

$617853-1000 \times 2391$

DAVID J FIRST

PRESIDENT

DJ INSTRUMENTS INCORPORAIED

I\& REPUBLIC RD

NOR TH BILLERICA. MA 01862

$617667-5301$ 
RAY FISCHER

PRODUCT COORDINATOR

BROCKWAY INC

BROCKWAY, PÁ 15824

$814268-3015$

HAROLD L FISHER

2113 SOUTH SANTA FE

OKL AHOMA CITY. OK 73109

405 235-9212

JAY-DONALD FISHER

RED-ROSE TRANSFER INC

1526 BILTMORE AVE

LANCASTER PA 17601

$717397-0953$

RONALD FISHER

WEIGHTS \& MEASURES RETAIL INSP 8 ENRIGHT AVE

WINDSOR , VT 05089

$802674-5355$

RONALD F FLEMING

TECHNICAL SERVICE MANAGER

MCCORMICK CO

1311 SCHILLING PLACE

SAL INUS, CA 93912

$408758-2411 \times 221$

JAMES C FLINT

PRODUCT MANAGER

NATIONAL CONTROLS INC

PO BOX 15012320 AIRPORT BLVD

SANTA ROSA CA 95402

$707527-5555 \times 284$

HERB N FLOAN

MANAGER

VIKING FEEDS INC

$305 \mathrm{~W}$ GRANT ST/PO BOX 804

DETROIT LAKES. MN 56501

$218847-4749$

ROBERT L FONGER

SEN IOR TECHNICION

BENNETT PUMP CO

2740 WOOD ST

MUSKEGON, MI 49444

$616733-1302$

ROGER H FORD

OFFICE MANAGER

RAY EQU IPMENT CO INC

5538 MERR I AM DR

AERRI AM, KS 66203

$913384-1070$

BARRY G FOSDICK

OWNER

PETROLEUM EQUIPMENT GMAINTENACE

PO BOX 53

BASSETT: WI 53101

$414877-2661$

MICHAEL FOSTER

REG ISTERED SERVICEMAN

KELLY OIL COMPANY

$B 0 \times 1016$

RAWLINS, WY 82301

$307324-3332$

BILL FOWLE

COLGATE PALMOLIVE

P 0 BOX 9

JEFFERSONVILLE. IN 47130

$812283-6611$
$J A M E S$ G FOX

HEINZ USA

PITTSBURGH,PA 15230

N E FRAILEY

PACKAGING COORD LUBRICANTS

SHELL OIL COMP ANY

$P 0$ BOX 2463

HOUSTON, TX 77001

$713241-2689$

AARON D FRANZBLAU

PRESIDEN T

ARKSTROM INOUSTRIES INC

415 AVON AVE

NEWARK. NJ 07108

$201243-1410$

ROBERT M FREEBURG

PRES

HUNTOON EQUIPMENT CO

624 LIVERNOIS

FERNDALE MI 48220

$313543-6900$

WILLIAM C FREY

VICE PRES TECHNICAL SER VICES BRISTOL-MYERS PRODUCTS

225 LONG AVE

HILLSIDE NJ 07207

201 926-9304

RONALO FREYER

MANAGER OUALITY GROCERY

PILLSBURY COMPANY

PILLSBURY CENTER

AINNEAPOLIS. MN 55402

HILLIAM S FULLER

PRE SIDENT

SYSTEMS SERVICES

$P$ O BOX 65

BREMEN, OH 43107

$614878-4269$

DAVID B FUNK

PROJECT DEVELOPMENT ENG INEER

D ICKEY-JOHN CORPORATION

155 S COUNTRY CLUB RD

AUBURN, IL 62615

$217438-3371$

G RICHARD FURBER

STAFF DISTRIBUTION REPR

SHELL OIL CO

TWO SHELL PLAZA-RM 1508

HOUSTON. IX 77001

$713241-4950$

RAYMOND GALANI

MANAGING EDITOR

FOOD CHEMICAL NEWS INC

1101 PENNSYLVANIA AVE SE

WASHINGTON: DC 20003

$202544-1980$

ADOLPH H GALINDO JR

DELTA SCALE INC

10108 WOODVIEW CIRCLE

MATTHEWS, NC 28105

$704527-8256$

PATRICIA GAMBLE

MANAGER PACKAG ING

W ATLEE BURPEE COHPANY

300 PARK AVE

WARMINSTER. PA 18974

$215674-4900 \times 255$ 
JERRY E GAMOTIS

MOB ILE INSTRUMENT CO INC

701 S CONCEPTION

MUBILE, AL 36603

ER IC A GARD

VP \& OPERATIONS MGR

PENNSYL VANIA SCALE CO

12 GRAYBILL AVE

LEOLA, PA 17540

$717656-2653 \times 42$

CHARLES H GARDNER

PRESIDENT

SERAPHIN TEST MEASURE

10 INDEL AVE

RANCOCAS, NJ 08073

$609267-0922$

GEORGE E GARDNER

PEPSI COLA CO

3 GANNETT DR 2ND FLOOR

WHITE PIAINS. NY 10604

TERRY C GARDNER

TECH SVCS COORDINATOR

CLOROX COMPANY

PO BOX 493

PLEASANTON CA 94566

JOHN E GARNSEY

GULF COAST REGIONAL SALES MGR

MAR TIN-DECKER

2630 FOUNIAINVIEN SUITE 410

HOUSTON. TX 77057

$713780-8787$

JAMES R GARRETT

MANAGER PRODUCT ENGINEER ING

HOBART CORPORATION

TROY, OH 45374

513 332-3000

G L GAR TEISER

MGR DISTRIBUTION E ENGINEERING EXXON COMPANY USA

$P$ B BOX 4388

HOUSTON. TX 77210

$713656-6113$

CARLION J GASKINS

OWNER

$J$ RED GASKINS \& CO

HWAY 52. SOUTH

LAKE CITY, SC 29560

803 394-8830

\section{R L GATES}

PLANT SUPERINTENDENT

UTAH INTERNATIONAL NAVAJO MINE $P$ O BOX 155

FRUITLAND, NM 87416

505 598-5861

IMOTHY R GAVIN

QUALITY ASSURANCE MANAGER

AMOCO FOAM PRODUCTS CO

2907 LOG CABIN DR

SMYRNA, GA 30080

$404351-5151$

RICHARD D GAYNOR

VP OF ENGR AND RESEARCH

NATL READY MIXED CONCRETE ASSN

900 SPR ING ST

SILVER SPRING. MD 20910

$301587-1400$
WILLIAM G GEME INER

SCALE ENGINEER

CHICAGO AND NORTHWESTERN TRANS

ONE NORTHWESTERN CENTER

CHICAGO, IL 60606

$317559-6133 \times 6610$

REX GEPFER T

PLANT MANAGER

FERRANTI INDIANA INC

4211 ENGLE ROAD

FT WAYNE: IN 46804

$219432-4214$

ROBERT D GERBER

ASSISTANT TO THE PRESIDENT

BLUE FLAME GAS CORP

PO BOX 376

BLUFFTON: IN 46714

219 824-3000

WALTER F GERDOM JR

MANAGER - TECHNICAL SERVICES

TOKHE IM CORPORATION

$P O B O X 360$

FORT WAYNE IN 46801

$219423-2552 \times 316$

MARY GETTY

OUALITY AS SURANCE DIRECTOR

ENTERPRI SE COMPANIES

1191 SOUTH WHEELING ROAD

WHE EL ING. IL 60090

$312541-9000$

ANGELO GIAMMAR INO

OUALITY ASSURANCE DIRECTOR

TENCO DIVISION OF TETLEY INC

$P$ O BOX 15

LINDEN, NJ 07036

$201862-6600 \times 283$

JOE GIANNINA

SUPR BULK MATERIALS DOCKS

PORT OF CORPUS CHRISTI AUTH

$P$ O BOX 1541

CORPUS CHR ISTI. TX 78403

$512882-1956$

THOMAS M GILMORE

TECHNICAL DIRECTOR

DAIRYIFOOD INDUSTRIES SUPPLY

6245 EXECUT IVE BLVD

ROCKVILLE. MD 20852

$301984-1444$

MICHAEL J GILROY

FOOD E DRUG SENIOR COUNSEL

COCA COLA COMPANY

$P$ O DRAWER 1734

ATLANTA, GA 30301

$404676-2121$

JOSEPH GINER

PRESIDEN T

HINSLOH SCALE COMPANY

PO BOX 1523

TERRE HAUTE, IN 47808

$812466-5265$

ROBERT M GODBOUT

QUALITY CONTROL

BERNAT YARN E CRAFT CORP

DEPOT $C$ MENDON ST

UXBR IDGE. MA OI569

$617278-2414$

HAROLD D GODSEY

MGR PROCESS ENGINEERING

THE OUAKER OATS CO

MERCHANDISE MART

CHICAGO. IL 60654 
MARK G GOEDDE

DIREC TOR OF ENG INEERING SVCS

NATIONAL GRAIN \& FEED ASSN

725 15TH ST NW PO BOX 28328 HA SHING TON, DC 20005
$202783-2024$

ALLAN J GOODFELLOW GENERAL COUNSEL AND SECRETARY AHERICAN GREETINGS CORPORATION 10500 AMER ICAN RD CLEVELAND。 OH 44144 $216252-7300 \times 1344$

HILLIAM Y GOODPASTER

VICE PRESIDENT

CARDINAL SCALE CO

$1610 \mathrm{~N} C \mathrm{C}$ ST

SACRAMENTO: CA 95814

$916441-0178$

GEORGE E GOODRICH

ENVIRONHENTAL \& SAFETY MANAGER

BURMAH CASTROL INC

401 HACKENSACK AVE

HACKENSACK, NJ 07601

$201488-1080 \times 62$

THOMAS G GORDON

OUALITY ASSURANCE MANAGER

MAR IN DECKER COMPANY

1200 CYPRESS CREEK ROAD

CEDAR PARK TX 78613

$512331-0411 \times 265$

HALTER J GOULET

SENIOR REGIONAL SALES

MARTIN-DECKER

4989 TURTLE ROCK DR

MAR IETTA, GA 30066

$404928-7051$

ROBERT A GRAHAM

TECHNICAL DIRECTOR

SPRAYON PRODUCTS

26300 FARGO AYE

BEDFORD HEIGHTS OH 44146

FRANK D GRAVES

PRESIDENT

ERNIE GRAVES COMPANY INC

2015 HOUSTON

IULSA, OK 74127

$918584-4707$

NORMAN A GREEN

VICE PRESIDENT

SGS CONTROL SERVICES INC

42 BROADWAY

NEW YORK. NY 10004

$212482-8700 \times 226$

DALE L GREENBANK

SUPPLY E DISTRIBUTION MGR UNI TED AMER I CAN FUELS

$P$ O BOX 16627

PHOENIX, A2 85011

$602253-8981$

BETTY F GREITZER

MANAGER-PUBLIC AFFAIRS

PATHMARK SUPERMARKETS

301 BLAIR RD

WOODBRIDGE NJ 07095

$201499-3424$

SCOTT GR IFF IN

MANAGER CONSUMER PROOUCTS RES

$U$ S BORAX RESEARCH CORP

412 CRE SCENT WAY

ANAHEIM, CA 92801
TERRY L GRIMES

SECRETARY

NATIONAL BUREAU OF STANDAROS

WASHING TON, OC 20899

JACK C GROSSKOPF

TECHNICAL DIRECTOR

FANTASY FL AVOR S INC

24 W 51 NORTH A VENUE

HHEATON, IL 60187

$312668-1000$

MARTIN R GRUBER JR

OIRECTOR OF SCALE TESTING

SOUTHERN WEIGHING 6 INSP BU

151 ELL IS ST NE $\$ 306$

ATLANTA. GA 30303

$404659-6266 \times 266$

STEVE GRULER

MANAGER QUALITY ASSURANCE

THE OUAKER OATS COMPANY

400 SECOND STREET N E

CEDAR RAPIDS, IA 52406

$319362-3121 \times 126$

RICHARD L GRUSKOS

VICE PRE SIDENT/ENG INEER ING

HOFFER FLOH CONTROLS INC

P O BOX 130

PORT MONMOUTH, NJ 07758

201 787-1997

JERRY GR ZESKOWI AK

MANAGER QUALITY CONTROL

NATIONAL OATS COMPANY INC

1515 H AVENUE NE

CEDAR RAPIDS. IA 52402

$319364-9161$

$S$ ROBERT GUINTER

CHIEF PRODUCT DESIGN ENGINEER

COUNSELOR COMPANY

2107 KISHWAUKEE ST

ROCKFORD, IL 61101

$815968-9621 \times 267$

PAUL GUNDERSON

I NDUS TR I AL PRODUC TS MANAGER

NAT IONAL CONTROLS INC

2320 AIRPORTT BLVD PO BOX 1501

SANTA ROSA. CA 95402

$707527-5555 \times 238$

JOHN D GUNN JR

VICE PRESIDENT

OHIO COUNTING SCALE NORTH INC

4541 INDUSTRIAL PARKWAY

CLEVELAND, OH 44135

$216941-4500$

R E GUNNER SON

DIRECTOR OUALITY ASSURANCE

SUN-DI AMOND GROWER S OF CALIF

1050 S DIAMOND ST PO BOX 1727

STOCK TON, CA 95201

$209466-4851$

DONALD HAGENBART

VICE PRESIDENT ENG INEER ING

WISCONSIN ELECTRICAL MFG CO

2501 SOUTH MOORLAND ROAD

NEW BERL IN. WI 53151

$414782-2340$

ROY HAIGH

SALES MANAGER

COMPETROL INC

8714 LION ST SUITE D

CUCAMONGA, CA 91730

$714987-0808$ 
KHAL IL D HAKER

$\checkmark P$ ENG INEERING

BLH ELECTRONICS

75 SHAWMUT INDUSTRIAL PARK

CANTON. MA 02021

$617821-2000$

\section{$M$ \ HAL BLE IB}

SEN IOR ENG I NEER

CHEVRON USA INC

P O BOX 7006 ROOM 2672
SAN FRANCISCO. CA 94120

DAVID E HAM

PROJECT PLANNING MANAGER

CHESEBROUGH-PONDS INC

33 BENEDICT PLACE

GREENWICH CT 06830

$203661-2000 \times 1358$

BRUCE H HAMILL

GENERAL COUNSEL

NATIONAL PAINT \& COATINGS ASSN

1500 RHODOE I SLAND A VE NW

WASHING TON, DC 20005

$202462-6272$

ROBERT M HAMILTON

PRESIDENT

INDUSTRIAL SCALES INC

RTE 5 BOX 99B I- 55 SOUTH

JACKSON. MS 39212

$601372-5384 \times 270$

BR I AN HAML IN

MASTER JOURNEYMAN

GREAT NORTHERN PAPER COMPANY $P 0$ BOX 240 WEST BR ANCH SHOP MILLINOCKET , ME 04462

$207723-5131 \times 1435$

JOHN R HAMMEL

HAMMEL SCALE COMPANY INC

WICHITA. KS 67214

GENE HAMMER

PRESIDENT

ACME SCALE \& SERVICE COMPANY

335 SOUTHHEST BLVD

KANSAS CITY, MO 64108

$816842-2731$

VIRGIL R HAMMOND

MAINTENANCE SUPER VISOR

114 E ARMOUR BLVD

KANSAS CITY, MO 64111

$913967-0133$

MICHAEL H HANEY

DIR REGULATORY AFFAIRS

ROSS LABORATORIES

625 CLEVELAND AVE

COLUMBUS. OH 43216

$614227-3212$

MELVIN C HANKEL

CHIEF DESIGN ENG INE ER

LIQUID CONTROLS CORPORATION WACKER PARK

NORTH CHICAGD, IL 60064

$312689-2400$

WILLIAM R HANNA

SIOUXLAND SCALE SERVICE INC

$1218 \mathrm{~N}$ LEWIS BLVD

SIOUX CITY. IA 51105
GARY G HANSEN

ENG INEER ING MANAGER

P ITNEY-BOHES INC

380 MAIN AVE

NORHALK. CT 06852

HENRY C HARBER S

PRES IDEN

ELECTRONIC SCALES INTERNATL

P O BOX 3259

SAN LUIS, CA 93403

$213579-3323$

GEORGE C HARE

PRODUCT MANAGER

$P$ O BOX 23099

MILWAUKEE. WI 53223

$414355-0400$

WILLIAM F HARR IGAN

PLANT MANAGER

MC CADAM CHEESE CO INC

12 ANNETTE

HEUVELTON, NY 13654

$315344-2441$

ROBERT J HARRINGTON

MGR SAFETY SECURITY MKTG OPS

MARATHON PETROLEUM CO

539 SOUTH MAIN ST

FINDLAY, OH 45840

$419422-2121$

HAROLD E HARRIS

PRES I DENT

HARRIS ASSOCIATES

$22 R$ TOWN \& COUNTRY

HOUSTON, TX 77024

$713465-8034$

RAYMOND G HARR IS

NATIONAL SALES MANAGER

AIRHAY SCALE MFG CO INC

10630 SO SANTA FE AVE

SOUTH GATE. CA 90280

$213563-5700$

STEVEN E HARRIS

DIRECTOR OF ENGINEER ING

MOS SCALE INTERNATIONAL

3180 REDHILL AVE

COSTA MESA. CA 92626

$714754-7841$

IACK HAR SHMAN

MANAGER PRODUCT DEVELOPMENT

DANIEL INDUSTR IES INC

PO BOX 19097

HOUSTON. TX 77024

$713827-5131$

DAVID B HARTLEY SR

ASST MGR OUALITY ASSURANCE

NESTLE FOODS CORP

100 BLOOMINGDALE ROAD

WHITE PLAINS. NY 10605

$914682-6857$

GORDON D HARTMAN

OIRECTOR CORP OUALITY CONTROL TROPICANA PRODUCIS INC

P O $80 \times 338$

BRADENTON, FL 33506

$813747-4461 \times 3220$

WILLIAM 8 HASTINGS

MGR OUALITY 6 FIELD SERVICES

INTERSTATE MILK PRODUCERS COOP

P O BOX 127

SOUTHAMP TON. PA 18966

$215322-0200$ 
WALTER HAUSHERR

DIR MGMT SERVICES

METTLER INSTRUMENT CORP

$B 0 \times 71$

HIGHTSTOWN NJ OB520

C HAWKINS

GR AVIME TRIC SPECI I I ST

WE IGHTS $\&$ MEASURES CCAC 4900 YUNGE ST-SUITE 601 WILLOWOALE M2N6BB

$416224-405 B$

GLENN W HAYES

PRESIOENT

VALLEY SCALE COMPANY

2306 BURBANK BLVO

BURBANK, CA 91506

$213841-0151$

RON HAYES

PETROLEUM LAB SUPERVISOR

MO DEPT OF AGRICULTURE

$P O B O X 630$

JEFFERSON CITY, MO 65102

$314751-2922$

RICHARD M HAYFORD

CONSULT ANT

UNION CARBIDE CORP LINDE DIV PO BOX 44

TONAHANDA, NY 14151

$716879-7207$

HARRY B HAYMES

SANDERS ASSOCIATES INC

95 CANAL ST

NASHUA NH 03061

$603885-4913$

PETER G HEACOX SR

OFFICE MANAGER

EAU CLAIRE EQUIPMENT CO INC 2620 DAVEY ST

EAU CLAIRE: WI 54703

$715 \quad 832-2987$

JOHN J HEALY

EXECUTI VE VICE PRESIDENT

GRAIN ELEVATOR \& PROCESSING

P O BOX 15026 COMMERCE STATION MINNEAP OL IS MN 554150026

$612339-4625$

DAVID M HEBENSTREIT

PRESIDENT

RIGHT WEIGH SCALE CO

RT $10 \mathrm{HWY} B O E$ AIRPORT IND PK

PEARL, MS $3920 B$

$601932-4790$

RAYMOND HEBENSTREIT

CONSULTANT

ACCURATE SUPERIOR SCALE CO INC 3747 S JEFFER SON

ST LOUIS. MO 63118

$314771-9359$

JOSEPH G HECK PHO

OUALITY AS SURANCE

KNUDSEN CORP

1900 WES I SLAUSON AVE

LOS ANGELES. CA 90047

213 744-7000

$R$ D HEER

MANAGER-TRANSIT \& AUDIT SERV

WESTERN HEIGHING \& INSP BUR

222 S RIVERSIDE PLAZA RM 1130

CHICAGO, IL 60606

$312559-0510$
LEE HEINRI CHS

CIBAF GEIGY CORPORATION

$P$ B BOX 11422

GREENSBORO, NC 27409

919292-7100

RAYMOND H HELMICK

MANAGER-WE IGHING SYSTEMS

PEAGODY COAL CO AR I ZONA DIV

$163 B$ E CINNABAR AVE

PHOENIX: AZ B5020

$602943-3837$

JOHN J HENKE

UNION CARBIDE CORPORATION

$P$ O BOX 2B31 BLDG B280

CHARLESTON: WV 25330

$304747-6200$

MAR ILYN J HERMAN

PRES IDENT

HERMAN AND ASSOCIATES

$2300 \mathrm{M}$ ST NW SUITE 800

WASHINGTON, OC 20037

MICHAEL HERMES

OWNER

WIDE RANGE SCALE SERVICE

991 BEARS DEN RD

YOUNGSTOWN, OH 44511

$216799-2617$

TROY E HERR ING

BRANCH MANAGER

G T MICHELLI CO INC

425 GUIDICI STREET

JACKSON. MS 39204

$601352-8 B 09$

JEFFREY $N$ HERSTEIN

DIRECTOR OF SALES

POLY-TECH INC

1401 WEST 94 TH STREET

MINNEAPOLIS, MN 55431

$612884-7281 \times 232$

STEPHEN J HIEBER

OWNER

PWI INCORPORATED

211 LINCOLNWAY W

NEW OXFORD. PA 17350

$717624-2111 \times 25$

E LEE HI GG I NBO THAM

AL ELECTRONIC CASH REGISTER 25 WEST OXMOOR RD

BIRMINGHAM, AL 35209

L KENNETH HILLER

HEAD REGULATORY REL FOOD DIV

PROCTER AND GAMBLE CO

CINCINNATI. OH 45224

$513977-7969$

LOUIS $K$ HILLER

REGULATORY RELATIONS - FOODS PROCTER AND GAMBLE

6083 CENTER HILL RD

CINCI NNATI, OH 45224

$513659-7291$

JAMES R HINCHEY

CHIEF BASIC STOS BRANCH

CAL IBRATION DIVISION OA-CB

U S ARMY WITE SANDS MISSILE RANGE N NM B8002

$50567 \mathrm{~B}-1720$ 
DONALD J HINE

PETROLEUM MEAS CONSULTANT

AEM COR PORATION

452 TENTH ST

ELYRIA. OH 44035

$216323-2041$

KARL HINRICHS

VP

NEWPORT ELECTRONICS

630 E YOUNG ST

SANTA ANA CA 92705

$714540-4914 \times 35$

PETER J HLAVAC

SALES MANAGER

GARSITE PRDDUCTS INC

10 GRAND BLVD P O BOX 289

DEER PARK, NY 11729

LEE HOCK

FIELD SERVICES COORDINATOR

STANDARD OIL COMPANY

4850 EAST 49 TH STREET

CLEVELAND. OH 44125

$216271-8211$

RICHARD L HOCKMUTH

MECHANICAL PRODUCTS ENGR MGR

PMP COR PORATION

25 SECURITY DRIVE POB 422

AVON, C 06001

$203677-9656$

$G$ F HOD SMAN

LEGAL METROLOGY EXECUT IVE

W \& T AVERY LTO

SMETHWICK WARLEY

WEST MI DLANDS

0213537693

H CARL HOEFER JR

PRODUCT MANAGER - F $3 W G$

MONSANTO

$800 \mathrm{~N} L$ INDBERGH

SI LOUI S MO 63166

KENNETH R HOFFER

PRESIDENT

HOFFER FLOW CONTROLS INC

$P O B O X 130$

PORT MONMOUTH, NJ 07758

$201787-1997$

DAWN M HOFFMAN

SECRETARY

NATIONAL BUREAU OF STANDARDS

WASHING TON. DC 20899

HAROLD L HOLDORF

AGENT

HOBART SALES $E$ SERVICE

2116 CLEVELAND AVE

ANCHORAGE. AK 99503

907 248-0700

EUGENE H HOLEMAN

STATE LIAISUN REPRE SENTATIVE

ASSN OF ANALYTICAL CHEMISTS

276 HARDING PLACE

NASHVILLE. IN 37205

$615352-3710$

CHARLES T HOLTGREVEN

PRESIDENT

HOLTGREVEN SCALE \& ELECT CORP

420 EAST LINCOLN ST

FINDLAY, OH 45840
E REPSTORFF HOLTVEG

DIRECTEUR

DANTEST

AMAGER BOULEVARD 115

ROBERT B HOOD

DIRECTOR OF ENGINEER ING

LOOEC INC

DRAWER D

LYNHOOD. WA 98036

$206775-6471$

BRIAN HOOVER

PRODUCT MARKET ING ENGINEER

7070 WINCHESTER CIRCLE

BOULDER. CO 80301

$303530-8534$

BARNEY T HOPKINS

MFG SERVICES SUPERVISOR

CLOROX COMPANY

$P$ B BOX 493

PLEASANTON CA 94566

LARRY $K$ HOPKINS

ELECTRICAL ENGINEER

GCLDEN REC YCLE CO

3000 YOUNGFIELD SUITE 230

LAKEWOOD CO 80215

$303277-5794$

DEBORAH J HORNER

OUAL ITY CONTROL MANAGER

THOMAS J LIPTON INC

2200 DELAHARE AVE

SANTA CRUZ CA 95060

$408426-9100 \times 0336$

RONALD J HOSTETLER

DENVER REGION SCALE INSPECTOR

BURLINGTON NOR THERN RAILROAD

$R R I B O X 100$

MILFORD, NE 68405

$402761-2623$

CLIVE A HOWARD-LUCK

DIRECTOR

CONSUMER TRADING STANDARDS DPT

STRATHCLYDE HOUSE INDIA ST

GLASGOW G24PF

HARR ISON N HOWE

MATERIAL HANDL ING MANAGER

MONSANTO COMPANY

$800 \mathrm{~N}$ LINDBERGH BL VD

ST LOUIS. MO 63166

$314694-5671$

ROGER HOWELL

SCALE TECHNICION

$\checkmark A N$ DYKE SCALE CO

2825 RAY IOWN RD

KANSAS CIIY. MO 64128

$816861-7191 \times 92$

ARTHUR HUBBARD

SER VICE MANAGER

OIL EOUIPMENT SALES $\angle$ SVC CO

P 0 BOX 5096

CHESAPEAKE, VA 23324

804 543-3596

HAL HUDGINS

GAR NAC GRAIN CO INC

$P$ O BOX 59

KANSAS CIIY. MO 64141

$816932-0140$ 
THOH HUELL INGHORST

ENG INEERING MANAGER INC

1326 ASHBY RD

$314991-024063132$

ARTHUR J HUETTEMAN

DIR REGULATORY AFFAIRS

PER SONAL PRODUCTS COMPANY

VAN LIEW AVE

MILLTOHN. NJ 08850

$201524-7294$

JAMES $K$ HUMBLE

DIRECTOR

NATL ME TROLOGICAL COORDIN UNIT PO BOX 6 FELL ROAO CROYDON

$$
01688 \quad \times 1996
$$

VERNON T HUMPHRIES

MAINTENANCE SUPVT

POLK OIL CO INC

1422 SOUTH 1 ST

LUFKIN. TX 75901

$409634-8484$

LARRY F HUNTER

MANAGER TECHNICAL SERVICES

SOUTHERN PUMP \& TANK CO

P O BOX 31516

CHARLOTTE, NC 28231

$704596-4373 \times 230$

DICK HURLEY

MGR REGUL COMPLIANCE

FAIRBANKS HEIGHING DIV COLT IN 711 EAST ST JOHNSBURY RD

ST JOHNSBURY, VT O5819

$802748-5111 \times 349$

ROBERT HUTCHINSON

ALPOPETFOODS INC $309 /$ POPE RD ALLENTOWN. PA 18001

FRANK S HYER

PRESIDENT

THAYER SCALE DIV HYER IND

RT 139

PEMBROKE: MA 02359

MARTIN A HYNES

HEAD-METROLOGY LABORATORY BALLYMUN ROAD

DUBLIN 9

RAY S IDZIOR

GEN MGR WEIGHING EQUIPMENT DIV

CMI COR PORATION

P O BOX 1985

OKL AHOMA CITY, OK 73101

$405787-6020$

SHARON L IRVINE

ASSOC ATTORNEY ADV/FOOD \& DRUG

KRAFI INC

GLENVIEW: IL 60025

$312998-2796$

C H I SLEY

DIRECTOR QUALITY AS SURANCE

GOODMARK FOODS INC

P O BOX 187

GARNER. NC 27529

$919772-1511$
MAT J JACOBCHICK

MANAGER ENGINEERING \& SERVICE

REXWORKS INC-CONST MACH DIV

445 OKLAHOMA AVE BLDG F2

MILHAUKEE HI 53207

$414643-2230$

W TERRY JAMES

VICE PRES ENG INEER ING SERVICES

CARDINAL SCALE MFG CO

203 E DAUGHERTY

WEBB CITY, MO 64870

$417673-4631 \times 20$

RALPH JENKS

PRESIDENT

CATO OIL AND GREASE COMPANY

$915 \mathrm{~N}$ EASSTERN

OKLAHOMA CITY, OK 73117

$405424-3311 \times 221$

ROGER JENNEY

CAL IBRAT ION COORDINATOR

FAULTLESS RUBBER CO

$268 W$ 4TH STREET

ÁSHLAND, OH 44805

KNUD E JENSEN

961 MC CLELLAND ST

SALT LAKE UT 84105

$801364-9350$

RANDY JENSON

MICRO MOTION INC

7070 WINCHESTER CIRCLE

BOULOER. CO 80301

$303530-8400$

ALFRED E JOHANSON

SENIOR COUNSEL

MCKESSON CORP LAW OEPARTMENT

155 EAST 44 ST

NEW YORK: NY 10017

JOHN T JOHNS

SCALE REPAIRMAN

OLIN CORP

SHAMROCK BLDG 246

EAST AL TON, IL 62024

$618258-2000 \times 2129$

CONRAD JOHNSON

MANAGER OUALITY ASSURANCE

COATS AND CLARK INC

$P$ BOX 670

TOCCOA. GA 30577
$404886-2141$

MARK O JOHNSON

230 W COLEMAN ST

RICE LAKE, WI 54868

$715234-9171$

RICHARD L JOHNSON

MANAGER QUALITY CONTROL

BEATRICE MEATS INC

1919 SWIFT DR

OAK BROOK, IL 605229010

$312850-2825$

ROBERT G JOHNSON

NASH FINCH COMPANY

3636 STOLLEY PARK ROAD

GRAND ISLAND. NE 68802

612 929-0371 
SPENCER A JOHNSON

VICE PRESI DENT

PAPERBOARD PACKAGING COUNCIL

1101 VERMONT AVE NW SUITE 411

WASHINGTON:DC 20005
$202289-4100$

WAYNE W JOHNSON

JOHNSON SCALE CO

P O BOX 1329

W CALDWELL. NJ 07006

JOHN A JOHNSTON

CARGILL INC

P O BOX 9300/CMD \#1

MINNEAPOLIS, MN 55440

$612721-8531$

DAVID K JOHNSTONE

TRAFFIC AND TRANS ENGINEER

UNI TED STATES STEEL CORP

600 GRANT STREET RM 460

PITTSBURGH PA 15230

$412433-3075$

JAMES JONES

VICE PRES IDENT

SHAMROCK SCALE COMPANY

5553 JEFFREY LANE/PO BOX 1719

MORRISTOWN: TN 37814

$615586-2083$

ROBERT E JONES

NATIONAL ACCOUNTS REPR

HOBART CORPORATI ON

5584 BANDINI BLVD

BELL. CA 90201

$213260-7321$

WIJUJOR DAN JJR

$J$ P STE VENS CO INC

P O BOX 2850

GREENVILLE SC 29602

GERRY A JOROWSKI

PROGRAM OFFICER

CONSUMER \& CORPORATE AFFAIRS

50 VICTORIA PLACE DU PORTAGE

HULL QUEBEC

PETER JUNG

SENIOR ENGINEER

BI ZERBA WERKE WILHELM KRAUT KG WILHELM KRAUTSTRASSE 41

D-7460 BALINGEN

CLARENCE T KASLINE

PRDOUC T MARKETING MANAGER

TOBAL DIV VERTEX INDUSTRIES

23 CAROL ST

CLIFTON, NJ 07014

$201473-6900$

\section{R J KATHMANN}

PROCTER \& GAMBLE MFG CO

1601 WEST 7 TH ST

LONG BEACH. CA 90813

FRED H KATTERHE INRI CH

MANAGER AGENCY/DESIGN POLICY

HOBART CORP

TROY
$5133 \mathrm{OH}^{4} 45374$
GENE I KATZ

VICE PRESIDENT

NAT IONAL CONTROLS INC

$P O B O X 15012320$ AIRPORT BL VD

SANTA ROSA CA 95402

ROBERT H KELLEN

PRESI DENT

ROBERT H KELLEN COMPANY

5775 D PEACHTREE DUNWODOY RD

ATLANTA. GA 30342

$404252-3663$

DANIEL A KENDRA

PRECISION WEIGHT SCALE CO

315 RICKERT ROAD

SELLERSVILLE. PA 18960

ROBERT L KENNEDY

PRESIDENT

MIDHEST SCALE COMPANY

1327 SEVETH ST

ROCKFORD, IL 61108

$815968-3731$

GLENN R KERLER

PRE SIDENT

FIELD SERVICES CORP

$P$ O BOX 413

CONIFER, CO 80433

$303838-2442$

JOE F KESSLER

SCALE INSPECTOR

CONRAIL

501 E MICHIGAN

JACKSON. MI 49201

$517789-6261$

HILLIAM D KEY

MANAGER OF ENGINEERING

TOKHEIM CORPORATION

PO $80 \times 360$

FT WAYNE IN 4680

$219493-2554 \times 345$

PAUL KHAN

PRE SIDENT

REGU-TECH ASSOCIATES INC

$158 W$ BOSTON POST RD/POB 717 MAMARONECK, NY 10543

$914899-0224$

MARY P KILCOYNE

DIRECTOR LEG \& REG INFO

SOAP E DETERGENT ASSN

475 PARK AVE

NEW YORK, NY 10016

$212725-1262 \times 21$

DON J KINDT

SCALE REPAIR SUPERVISOR

JOHN MORRELL

1400 NOR THWEBER PO $80 \times 1266$

SIOUX FALLS. SD 57117

$605338-8200 \times 223$

JOSEPH B KIPPENBROCK

XIPPENBROCK SCALE SERVICE

2408 PLEASURE HOUSE RD

VIRGINIA BEACH, VA 23455

D E XITCHEN

OPERATIONS ENGINEER

UNION OIL OF CALIFORNIA

$P$ BOX 7600

LOS ANGELES. CA 90051

$213977-6108$ 
ROGER D KITCHING

PACKAGING MGR - CONSUMER PROD CROWN ZELLERBACH CORP

ONE BUSH ST

SAN FRANCISCO, CA 94104

$415951-5568$

BOB KLEESPIE

OWNER

KLEESPIE TANK \& PETROLEUM EQ P O BOX 106

MORRIS. MN 56267

$612589-2100$

TOM KLEVAY

VICE PRESIDENT

MILLERS NATIONAL FEDERATION

600 MAR YLAND AVE STE 305 WEST

WASHINGTON, DC 20024

$202484-2200$

CHIP KLOOS

$L A B$ MANAGER $-R \& D$

BEATRICE GROCERY GR OUP

$1645 \mathrm{~W}$ VALENC IA DR

FULLERT ON CA 926333899

$714680-1098 \times 1098$

E WILLIAM KNAUSS

VICE PRESIDENT

E W KNAUSS E SON INC

QUAKERTOWN. PA 18951

$215 \quad 536-4220$

GLENN C KNUDSEN

PACKAGING MANAGER

PHILIP A HUNT CHEMICAL CORP

5 GARRET MOUNTAIN PLAZA

WEST PATERSON, NJ 07424

$201944-4000$

KESAKATSU KO IZUMI

MANAGING DIRECTOR

JAPAN MEASUR ING INSTRUMENT FED 25-I NANDO-CHO SHIN JUKU-KU

TOKYO

RUDOLPH J KOLACI

PRESIDENT

TOTALCOMP INC

3-O9 SADDLE RIVER ROAD

FAIR LAWN, NJ 07410

$201797-2718$

JOHN K KOLB

QUALITY PUMP \& ELECTRIC INC $P O B O X 2621$

ROCK SPRINGS. WY 82901

\section{STEPHAN A KORT}

PRESIDENT

$J$ B DEE \& CO INC

$1722 \mathrm{~W} 16 \mathrm{TH}$ ST

INDIANAPOLIS, IN 46202

$317635-5548$

JOE KRAL

MANAGER

MORTEC INDUSTRIES INC

515 INDUSTRIAL PK RD POB 977

BRUSH, CO 80723

$303842-5063$

ART KROLL

MANAGER REGULATORY AFFAIRS

$G$ ILBARCO INC

7300 W FRIENDLY AVE

GREENSBORO, NC 27420

$919292-3011 \times 255$
ROBERT E KROLL

VICE PRESIDEN

INT ER COMP COMPANY

2520 XENIUM LANE

PLYMOUTH. MN 55441

$612559-0646$

GERRY KRUEGER

LOUIS DREYFUS CORPORATION

24 RICHMOND HILL AVE

STAMFORD, CT 06904

$203357-8444$

JOHN R KUKUCKA

$\checkmark$ ICE PRESIDENT ENG INEER ING SUBURBAN PROPANE GAS CORP

RT 10 PO BOX 206

WHIPPANY NJ 07981

$201887-5300 \times 366$

WAL TER E KUPPER

MANAGER ENG INEER ING

MET TLER INSTRUMENT CORP

BOX 71

HIGHTSTOWN NJ 08520

WAL TER P KUSHMUK

MGR ENGINEERING \& RESEARCH

CONTINENTAL SCALE CORPORATION

7400 WEST $100 T H$ PLACE

BRIDGEVIEH, IL 60455

$312598-9100 \times 34$

CARL R KUTELIS

DIRECTOR OF ENGINEERING

REVERE CORP OF AMERICA

$P$ D BOX 56

WALLINGFORD, CT 06492

ROBERT LA GASSE

EXECUTIVE DIRECTOR

NATIONAL BARK PRODUCER S ASSN

13542 UNION VILLAGE CIRCLE

CLIFTON, VA 22024

$703830-5367$

TED R LABREE

TECHNICAL MANAGER REG SERVICES R IVIANA FOODS

1702 TAYLOR STREET

HOUSTON. TX 77007

$713529-3251 \times 854$

ALICE W LACY

INLAND NORTHWEST SCALE CO INC PO BOX 11355 PARKWATER STA SPOKANE, WA 99211

ANTHONY J LADD

CONSULTANT

A J LADD WEIGHINGEPKGING SYSTM 255 N PORTAGE PATH SUITE 213

AKRON. OH 44303

$216836-4569$

JOSEPH M LAINO

CORP DIR OA $E$ REGULATORY COMPL

DCA FOOD INDUSTRIES INC

919 THIRD AVE

NEW YORK. NY 10022

$212371-6600 \times 300$

JAMES F LAMB

OWNER

MINNKOTA METER COMPANY

190 3RD ST NE

WATERTOWN, SD 5720

$605882-2270$ 
JOHN H LANDVATER PRESIDENT

LANDVATER ASSOCIATES

P O BOX 654

SUMMIT: NJ 07901

$201273-8757$

ROGER LANE

LANE E ANDERSON INC

7479 LEMHI ST

$208376-4300709$

WAYNE A LATIMER

SENIOR MEASUREMENT ENGINEER MAPCO INC

$1 B 00 S$ BALTIMORE ST

TULSA: OK 74101

$918599-3635 \times 635$

LAWRENCE J LAUER

GENERAL MANAGER

PAR-TEE COMPANY

STATE HIWAY \#1

SPENCER VILLE, IN 46788

JOSEPH L LAWRENCE

PACKAGE ENGINEER ING MANAGER

BR ISTOL-MYERS PRODUCTS

225 LONG AVENUE

HILLSIDE, NJ 07207

M L LAZEAR JR

MANAAGER REGULATORY AFFAIRS

$J M$ SMUCKER CO

STRAWBERRY LANE

ORRVILLE, OH 44667

ROBERT A LE CAIRE

OIRECTOR-LABORATORY SERVICES PRESTO PRODUCTS INC $P$ BOX 2399

APPLETON WI 54913

$414739-9471$

OAVIO P LEAHY

TECHNICAL CONSULTANT

KROGER CO

2 CAMPBELL ORIVE

HI GHL AND HE IGHTS, KY 41076

$606572-2200$

\section{CLYDE E LEE}

CIVIL ENGINEERING ECJ 4.2

UNIV OF TEXAS AT AUSTIN

AUSTIN, TX 78712

$512471-4549$

LOYAL W LEITGEN

MANAGER - TWINE DEPT

UNIVERSAL COOPERATIVES INC

7801 ME TRO PARKWAY

MINNEAP OL IS. MN 55420

$612854-0800 \times 182$

WOL FGANG LEMME

MANAGER TECHNICAL SERVICES

METTLER INSTRUMENT CORPORATION

PR I NCETON-HIGHTSTOWN ROAO

HIGHTSTOWN NJ 08520

$609448-3000 \times 221$

T A LESNIOWSKI

SUPERVI SOR PLANT TRAFFIC HOOKER CHEMICALS \& PLASTICS CO $P$ O BOX 159

NI AGARA FALLS, NY 14302
$716278-7622$
ROBERT A LESTER

ATTORNEY

COCA-COLA COMPANY

PO ORAWER 1734

ATLANTA. GA 30301

$404676-2530$

BARBARA LEVI ESO

ELIZABETH ARDEN INC $52 N D$ ST

NEW YORK, NY 10022

$212407-1112$

BOBBY K LEWIS

OWNER

BLUFF CITY SCALE COMPANY

2856-14 LAMBS PLACE

MEMPHIS, TN 38118

$901365-1194$

JOSEPH 8 LEWIS

MGR QUALITY EASTERN STATES

SHASTA BEVERAGES INC

6085 BARFIELD ROAO SUITE 204

ATLANTA. GA 30328

VIRGINIA M LEY

SR OUALI IY ASSURANCE TECHNOLG

$H$ \ HEINZ CO

$P O B O X 57$

PITTSBURGH, PA 15230

$412237-5214$

JONATHAN A LIBBY

MANAGER-QUALITY AS SURANCE

MONTEREY MUSHROOMS

$P$ O BOX 24

WAT SONVILLE, CA 95077

$408728-145 i \times 0242$

JACK A LIERLY

SUPERVISOR CORROSION \& MEAS

CONTINENTAL PIPELINE CO

7666 E 6IST SUITE 325

TULSA. OK 74133

9 $18743-8803$

GARY W LINOLEY

PRODUCTIION SUPERVISOR

MONSANTO CO

$P O B O X 473$

MUSCATINE. IA 52761

$319263-0093 \times 275$

GEORGE A LINTON

CONSUL TANT

GEORGE A LINTON INC

$1 B O B$ CANTON ROAO

BIRMINGHAM, AL 35216

$205823-2855$

ROY E LITTLEFIELD

EXECUIIVE DIRECTOR

GREATER WASHINGTON MARYLAND

9420 ANNAPOLIS RO SUITE 307

LANHAM. MO 20706

$301 \quad 577-2875$

RAYMONO J LLOYO

EXECUTIVE DIRECTOR

SCALE MANUFACTURERS ASSN

152 ROLLINS AVE SUITE 208

ROCKVILLE, MO 20852

$301984-9080$

HARRY E LOCKERY

HOTIINGER BALOWIN MEASUREMENTS 139 NEWBURY ST

FRAMINGHAM. MA 01701

$617875-8282$ 
N RICHARD LOCKMILLER

MANAGER GOVERNMENT RELATIONS STALEY CONTINENTAL INC $P$ O $80 \times 2513$

DECATUR, IL 62525

$217421-306$

HARVEY M LODGE

DUNBAR MANUFACTUR ING INC

307 BROADHAY

SWANTON OH 43558

$419244-3021$

SAM D LOFTIS

VICE PRES SALES \& MARKET ING NEPTUNE MEA SUREMENT COMPANY $P$ O BOX 792

GREENWDOD, SC 29648

RICHARD LOO VP ENGINEERING \& CONSTRUCTION GASCO INC PO BOX 3379

HONOLULU. HI 96842

$808547-3570$

CLAUDIA JLLUIS

MANAGER REGULATORY AFFAIRS

NATIONAL SOFT DRINK ASSOCIATIO

110116 TH STREET NH

WASHINGTON, DC 20036

$202463-6732 \times 6729$

LAHRENCE L LOWERY

GENERAL SCALE INSPECTOR

NORFOLK \& WESTERN RAILHAY CO

$8 \mathrm{~N}$ JEFFER SON ST

ROANOKE V VA 24042

F JOE LOYD JR

SUPERVISOR SCALES \& WEIGHING SEABOARD SYSTEM RAILROAD P O BOX 45052

JACKSONVILLE, FL 322325052

$904359-1024$

BERND G LUDEHIG

GEN MGR

BR I NKMANN INSTRUMENTS CO

CANIIAGUE RD

WESTBURY, NY 11590

ROY H LUTLEY

VICE PRESIDENT GENERAL MANAGER

EXACT WEIGHT SCALE

MI SSISSAUGA ONTARIO

$416625-6220$

DONALD L LYNCH

STANDAR DS ADMINISTRATOR

WEIGHTS AND MEASURE S CONTROL

710 NORTH 17 STREET

KANSAS CITY, KS 66102

$913342-5243$

JOHN MACDONALD

DIRECTOR OF ENG INEER ING

HOWE RI CHAROSON SCALE COMPANY

680 VAN HOUTEN AVENUE

CLIFTON, NJ 07015

$201471-3400$

GLENN E MACE

MGR OF OPER ATIONS-ENGINEER ING

BUCKEYE GAS PRODUCTS CO

7130 SOUTH LEHIS PO BOX 3478

TULSA, OK 74101

$918496-7535$
JAMES A MACFARLANE

VICE PRESIDENT

AUTOWEIGH CO

PO BOX 4017

MODESTO, CA 95350

$209526-3557 \times 9$

JOHN A MACINTOSH

VIDAL SASSOON INC

9640 OHENSMOUTH AVE

CHATWORTH, CA 91311

GERARD 8 MACK

PRODUCT ION SER VICES

DETROIT EDISON A-318 WSC

2000 SECOND AVE

DETROIT. MI 48226

$313897-0115$

BILL MAHLER

ELECTRONIC SYSTEMS ENGINEER

EMARK CORPORATION

11065 SORRENTO VALLEY CT

SAN DIEGO, CA 92121
$619457-1180 \times 312$

WAYNE N MAHONEY

INSPECTOR

MAR ION COUNTY AUDITORS OFFICE

COURTHOUSE ROOM 5

MAR ION, OH 43302

$614387-5871$

KENNETH MAIETTA

QUALITY CONTROL

TETLEY INC

$P$ D BOX 3337

WILLIAMSPORT, PA 17701

717 323-6133

HILLIAM C MAILHOT

DIRECTOR OUALITY CONTROL

GENERAL MILLS INC SPERRY DIV 9200 HAYZATA BLVD

MINNEAPOL IS. MN 55426

$612540-2354$

GEORGE MAJORS

MANAGER

HALVERSON COMPANY

235 WEST PAXTON AVE

SALT LAKE. UT 84101

$801467-9423$

\section{JOHN M MANN}

SEN IOR COUNSEL

US BORAX

3075 WILSHIRE BLVD

LOS ANGELES. CA 90010

$213381-5311 \times 1534$

JAMES J MANNING

PURCHASING AGENT

HM DI XON CO

750 WASHINGTON AVE

CARLSTADT NJ 07072

$201939-6700$

JAMES C MARAS

QUALITY ASSURANCE

MASTER BUILDERS

23700 CHAGRIN BLVD

CLEVELAND, OH 44122

$216831-5500$

PATRICK MAR INO

TECHNICAL SERVICE MANAGER

NEW BRUNSWICK INIERNATL INC

5 GREEK LANE

EDISON. NJ 08817

$201287-2288$ 
LYNN A MARVEL

ENG INEER ING MANAGER

CMI HEIGHING EQUIPMENT

$6101 W$ RENO SUITE 800

OKLAHOMA CITY, OK 73127

405 495-6830 ×308

WILLIAM J MASCOTT

PRESIDENT

MA SCOTT EQUIPMENT COMPANY INC

435 NE HANCOCK ST

PORTLAND, OR 97212

$503282-2587$

LEONARD MATLOSZ

VICE PRESIDENT

EASIERN SCALE COMPANY INC

1053 PENNSYLVANIA AVE

IINDEN. NJ 07036

$201486-4433$

\section{BOGDAN M MATOGA}

ENG INEER ING MANAGER REG COMPL NATL SEMICDNO-DATACHECKER/OTS PO BOX 58112

SANTA CLARA, CA 950528112

$408 \quad 982-3369$

ALLEN W MATTHYS

DIRECTOR REGULATORY AFFAIRS

NATIONAL FOOD PROCESSORS ASSOC

1401 NEH YORK AVE NH

WASHING TON, DC 20005

$202331-5960$

ROGER $L$ MAXON

BER NZOMATIC CORPORATION

BERNZOMATIC DIV NEWELL COS

ONE BERNZOMATIC DR

MEDINA, NY 14103

\section{GLEN MAYS}

MANAGER OF SERVICE

MEA SURE GRAPH CO

1694 LARKIN WILLIAMS ROAD

FENTON MO 63026

$314533-7800 \times 226$

TERRELL L MAYS

DIRECTOR PACKAGING

STAUFFER CHEMICAL COMPANY

HESTPORT, CT 06881

$203222-3390$

RICHARD R MC CALL

NATL DIR ENV HEALTH \& SAFETY

A E P TEA COMPANY

2 PARAGON DR

MONTVALE, NJ 07645

$201573-9700 \times 2577$

JOHN R MCCLURE

OUALITY ASSURANCE MANAGER

QUAKER OATS COMPANY

PO BOX 1677

CHATTANOOGA, TN 37401

$615698-1591 \times 178$

GARY R MCDONALD

OWNER

MASTER SCALES

713 HWY 82 WEST

GREENWOOD, MS 38930

$901346-4619$

ROBERT A MCDONALD

SERVICE MANAGER

P O BOX 1744

CUMBERLAND, MD 21502

$301759-3333$
PHILIP J MCGOWAN

MANAGER OF OUALITY ASSURANCE TETLEY INC

W RUBY E GRAND AVE

PALISADES PARK, NJ 07650

201 943-0600

TIM MCGUIGAN

BRANCH MANAGER

PETROLEUM CONTRACTORS INC RD 6 BOX $579 F$

ALLENTOWN. PA 18106

$215398-0421$

WILLIAM J MCHALE

VICE PRESIDENT

KANAWHA SCALES $E$ SYSTEMS ROCK BRANCH INDUSTRIAL PARK POCA. WV 25159

$304755-8321$

JOHN J MCINTYRE

PRESIDENT

JOHN J MCINTYRE SONS INC

514 KNORR

PHILADELPHIA. PA 19111

$215745-3304$

CAROLYN K MCKENZIE

SECRETARY

NATIONAL BUREAU OF STANDARDS

WASHINGTON. DC 20899

W J MCKNIGHT

MANAGER TECHNICAL SERVICES

KAL KAN FOODS INC

3376 E 44 TH STREET

VERNON. CA 90058

$213587-2727$

ROBERT A MCLEOD

ACTING DEP DIR AUDITS-SCALES CANADIAN GRAIN COMMISSION $800-303$ MAIN ST WINNIPEG MAN

\section{LEE D MCMAHAN}

PRESIDEN T

TRI-GAS \& OIL CO INC

$P$ O BOX 465

FEDERALSBURG. MD 21632

$301754-8184$

JOHN R MCPHERSON

SENIOR STAFF ENGINEFR

EXXON CO USA

$P$ BOX 4388

HOUSTON. TX 77210

$713874-5203$

RON MCVEY

PACKAGING/LABELING SPECIALIST

4900 YONGE ST SUITE 601

WILLOWDALE M2NGBB

JEROME F MELLO

PRESI DENT

NORTHEASTERN-PETROLFUA SERGSUP

37 BROOKLEY RO

BOSTON. MA 02130

$617522-8390$

DOUGLAS D MELOTI

OUALITY CONTROL MANAGER

KNUDSEN CORP

415 XANSAS AVE/PO BOX 5018

MOOESTO. CA 95352

$209575-5100 \times 5123$ 
GERALD E MERICLE

MANAGER $-R \& R$ TECHNOLOGY

REYNOLDS METALS CO

1803 REYMET ROAD

RICHMOND, VA 23237

$804743-6757$

WALTER MERKH

VICE PR ESIDENT ENGINEERING

PEABERTON FABRICATORS INC

30 INDEL AVE

RANCOCAS, NJ 08073

$609267-0922$

SONIA D MEURER

SR LIBR AR I AN

NAB ISCO BRANDS

PÜ BOX 1942

EAST HANOVER, NJ 07936

$201797-6800 \times 338$

G J MEYER

1416 DODGE ST ROOM 1000
UNION PACIFIC RAILROAD

1416 DODGE ST ROOM 1026

OMAHA, NE 68179

$402271-3153$

CHARLES W MICHELL JR

SR LOSS CONTROL REPRESENTATIVE SHELL OIL COMPANY

TSP ROOM 1140

HOUSTON, TX 77001

$713241-0455$

G T MICHELLI JR

PRESIDENT

$G$ I MICHELLI CO INC

$10116 \mathrm{HYDE}$ PLACE

RIVER R IDGE, LA 70123

$504885-9710$

DUANE MIKKELSEN

DOCKENDORF EQUIPMENT CO INC

$830 \mathrm{E} 15 \mathrm{TH}$ ST/PO BOX 1222

SIOUX FALLS, SD 57101

BRUCE A MILLER

ALLEN MILLER SCALES

5926 MANN ST

$216875-4179$ OH 44641

RICK E MILLER

OUALITY CONTROL SUPERVISOR

W M BARR \& CO

2105 CHANNEL AVE

MEMPHIS TN 38101
$901775-0100 \times 56$

RALPH W MILLER JR

COMMISSIONER INDUSTRIAL COMMN

STATE OF ILLINOIS

100 W RANDOLPH SI STE 241-C

CHICAGO, IL 60601

$312793-6607$

DONALD E MILLS

DISTRIC T SALES MANAGER

VEEDER-ROOT COMPANY

PO BOX 1673

AL TOONA PA 16603

$814695-4476 \times 25$

ART MI SENER

OC MANAGER

BEATRICE GROCERY GR OUP

$3327 \mathrm{~W} 47 \mathrm{TH} P$

CHICAGO, IL 60632
JOHN R MITCHELL

11336 WEMBLEY ROAD

LOS ALAMITOS. CA 90720

$213432-6981 \times 264$

THEEDSMUIR C MITCHELL

STANDARDS SCIENTIFIC OFFICER

JAMAICAN BUREAU OF STANDARDS

6 WINCHESTER RUAO KINGSTON 10

KINGSTON

$8099263140 \quad \times 217$

DARREL D MOATES

STATISTICAL TECHNOLOGY MANAGER KRAFT INC

KRAFT COURT

GLENVIEH, IL 60025

BRADFORD MOLNAR

DES I GN ENG INEER

FRANKLIN ELECTRIC CO

RT 2 BOX 1

WHITEVILLE, NC 28472

$919642-5913 \times 29$

THEODORE $F$ MOONEY

PRE S I DENT

AMER ICAN CALIBRATION/TEST INC

178 HALNUT ST

LAWRENCE, MA 01841

$617686-8652$

ANDREW B MOORE

SCIENCE AS SOC I ATE

GROCERY MANUFACTURERS OF AMER

1010 WISCONSIN AV NH STE 800

WASHINGTON, DC 20007

$202337-9400$

ANDREW 8 MOORE

QUALITY ASSURANCE AUDITOR

CASTLE E COOKE INC

$P O B O X 5130 / 100$ PARK PLAZA CT

SAN JOSE. CA 95150

$408279-8750 \times 4305$

$G$ E MOORE

$\checkmark$ P ENGINEERING

DOVER CORP OPH DIVISION

$P$ O BOX 405003

CINC INNATI, OH 45240

$513870-3208$

MARK MORE

PACKAGING \& PRODUCT COMPLIANCE

MCCORMICK \& CO

11350 MCCORMICK ROAD

HUNT VALLEY. MD 21031

$301667-7442$

ROBERT J MORRIS

VP ADVERTISING \& PUBLICATIONS

FEL-PRO INCORPORATED

$7450 \mathrm{~N}$ MCCORMICK BLVD

SKOKIE. IL 60076

$31276 i-4500$

DONALD G MORRISON

PRES

MORRISON WEIGHING SYSTEMS INC

INDIAN BLUFF RD BOX 860

MILAN. IL 61264

$309799-7311$

THOMAS L MORROW

MANAGER

MARKETING SPECIAL PROJECTS

19250 VAN NESS AVE

TORRANCE CA 90501

$213320-8900 \times 67$ 
FRANK A MOSEBAR PRESIOENT DFA OF CALIFORNIA

P O BOX $270 A$

SANTA CLARA: CA 95052

$408727-9302$

JAMES $\checkmark$ MOTS INGER

PRESIDENT

AMEACON INC

13703 THERMAL DR

AUSTIN, IX 78728

$512251-3483$

JOHN D MOYER

OUALITY CONTROL MANAGER

PLASTICS INC

$P$ O BOX 43440

SI PAUL. MN 55164

GEORGE A MUCK

VICE PRESIDENT RESEARCH \& DEV DEAN FOODS COMPANY

1126 KILBURN AVE

ROCKFORD. IL 61101

$815962-0647 \times 294$

BARBARA J MUELLER

MANAGER US OPERATIONS

SWEDA INTERNATIONAL INC

34 MAPLE AVE

PINE BRDOK, NJ 07058

$201575-8100 \times 303$

BRUCE A MURPHY

VICE PRESIDENT MARKETING

A H EMERY COMPANY

$P$ C BOX 608

NEW CANAAN CT 06840

ROBERT MURPHY

SERVICE MANAGER

RETAIL DATA SYSTEMS

106 GREYSTONE AVE

KANSAS CITY, KS 66103

$913281-1333$

JOSEPH R MURPHY JR

MARKETING MANAGER

EXXON CHEMICAL AMER ICAS

P 03272

HOUSTON. TX 77001

$713870-6178$

LARRY MURRAY

CHIEF ENG INEER

WAYNE DIV-DRESSER INDUSTRIES

124 W COLLEGE AVE PO BOX 1859

SALISBURY. MD 21801

$301546-6690$

ROBERT MURRAY

REGULATORY AGENCY COOROINATOR

BERKEL INC

ONE BERKEL ORIVE

$219326-7000$

CHUCK MYERS

MICRO MOTION INC

7070 WINCHESTER CIRCLE

BOULOER, CO 80301

$303-530-0530$

EARL $D$ MYERS II

SENIOR ENG INEER

OHAUS SCALE CORPORATION

29 HANOVER ROAD

FLORHAM PARK, NJ 07932

$201377-9000 \times 283$
VICKI NASH

QUALITY CONTROL MANAGER

CASE SHAYNE CO INC

1632 E $4 \mathrm{TH}$ ST

SANTA ANA. CA 92702

$714558-8861$

JOHN NAT I SHAN

DIRECTOR QUALITY CONTROL

PFI LER INC

100 JEFFERSON ROAD

PARSIPPANY. NJ 07054

WILLIAM C NEAL

VICE PRESIDENT-MARKETING

POL Y-AMER ICA INC

2000 W MAR SHALL

GRAND PRAIRIE. TX 75051

$214647-2950$

ROBERT L NELSON

MGR PHYSICAL TESTING-BAKING SE GENERAL MILLS INC

9000 PL YMOUTH AVE N

MINNEAPOLIS: MN 55427

$612540-2729$

MIL TON E NE SHEK

ASST SECY \& GEN COUNSEL

KIKKOMAN FOOOS INC

P O BOX 291

ELKHORN, WI 53121

HUGH WNESS

HAROY INSTRUMENTS INC

3565 CORPORATE COURT

SAN DIEGO CA 92123

RICHARD M NEU

O A TECHNOLOGIST

$S$ C JOHNSON E SON INC

1525 HOWE ST MS 273

RAC INE. WI 53403

$41463 i-3713$

JOHN L NEVEAUX

DIRECTOR REGULAIORY SFR VICES

MINNE TONKA INC

JONATHAN INOUSTRIAL CENTER

CHASKA. MN 55318

$612448-4181 \times 260$

MALCOLM T NEWELL

VICE PRES IOENT OPERATIONS

NORTHEAST PETROLEUM CORP

295 EASIERN AVE

CHELSEA. MA O2I50

$617884-7570$

ROBERT J NEWMAN

VP-MANUF AC TUR I NG

CONAGRA INC

ONE CENTRAL PARK PLALA

OMAHA. NE 68102

$402978-4050$

GLEN W NEWTON

MANAGER TRANSPORTAIION

ARCO COAL SALES COMPANY

555 SEVENTEENTH ST

DENVER, CO 80202

303 293-7785

R C NICHOLAS

A E N ASSOCIAIES

BOX 138A ROUTE I

LOVINGSTON. VA 22949 
NICKI NICOL

PRESIDENT

NICOL SCALES INC

DALLAS, IX 75222

$214428-8181$

WILIAM F NICOL

CHAIRMAN OF BOARD AND CEO

NICOL SCALES INC

PO BOX 222288

DALLAS. TX 75222

$214428-8181$

HOWARD A NIELSEN

CONSULTANT

2540 PAGE DRIVE

AL TADENA, CA 91001

$714739-1991$

J FRITS NIEMOLLER

PRESIDENT

JOHN CHATILLON \& SONS INC

8330 KEW GARDENS RD

KEH GARDENS, NY 11415

$212847-5000 \times 12$

$\checkmark$ NIEUHLAND

CHIEF LEGAL/JURIDICAL AFFAIRS DUTCH METROLOGY SER VICE

B $80 \times 654$

2600 AR

015569271

GRANT $V$ NI VEN

SYSTEM FUEL SUPER VI SOR

A T E S F RAILHAY

1001 E ATCHISON

$913235-0041 \times 7203$

JAMES M NOBLE

PRESIDENT

SELTEC CORPORATION

4568 ENTERPR ISE ST

FREMONT, CA 94538

$415657-7800$

WILLIAM NOHR

MGR FOOD PACKAGING SERVICE

WELLOTRON CORP

1532 S WASHINGTON AVE AT $1-287$

PISCATAHAY. NJ 08854

$201752-6700 \times 372$

RICHARD L NOLAN

QUALITY CONTROL MANAGER

62 WHI TTEMORE AVE

CAMBRIDGE, MÁ O2140

$617876-1400$

THOMAS L NOLAN JR

PRESIDENT

SANITAR Y SCALE COMPANY

910 E LINCOLN AVE

BELVIDERE, IL 61008

$815544-2181$

FRANK J NOSEK

VICE PR ESIDENT

KESON I NDUSTRIES INC

28 H 715 MOUNT ST

HARRENVILLE, IL 60555

$312393-3232$

KENNETH NUGENT

DISTR IBUTION ENG INEER

AIRCO INDUSTRIAL GASES

FOSTORIA. OH 44830
RICHARD T O CONNELL

PRE SIDENT

CHDCOLATE MANUFACTURERS ASSN

7900 WESTPARK DR

MCLEAN, VA 22102

$703790-5011$

DAN O DONNELL

SERVICE MANAGER

$P$ BOX AK

DES MOINES, IA 50302

Tा5 265-1661

CONOR O DONOGHUE

PRESIDENT

SHAMROCK SCALE COMPANY

5553 JEFFREY LANE/PO BOX 1719

MORRISTOWN: TN 37814

$615586-2083$

BILL O'NEIL

GASTOWN

23660 MILES AVE

BEDFORD HEIGHTS, OH 44128

$216475-9212$

DAVID M ODAY

NATIONAL SALES MANAGER

GOLDEN RECYCLE COMPANY

3000 YOUNGFIELD ST SUITE 230

LAKEWOOD CO 80215

303 277-5771

MARTIN E OEHLER

PRESIDENT

CHARLOTTE SCALE CO INC

1510 AMERON DR IVE

CHARLOTTE, NC 28206

704 376-8496

RICHARD E OGREN

PRESIDENT

SPECIALTY FOOD EOUIPMENT CO

306 DELAHARE

KANSAS CITY: MO 64105

$816842-1773$

TOM M OKON

PRE SIDENT

400 N I 4 TH ST P O BOX 1288

400 N 14 TH ST P 0

$517>55-5327$

ROGER H OLDS

PRE SIDENT

B-P METER TESTING CO INC

608 NORTHS IDE DR

ENTERPR I SE, AL 36330

$205347-8247$

MARK A OLSBERG

SENIOR PROJECT ENGINEER

BJ-TITAN SERVICES

11211 WEST FM 2920

TOMBALL. TX 77375

$713351-8131$

P T OLSON

PLANT ENGINEER ING MANAGER

MOB IL OIL CORPORATION

3225 GALLOWS RD ROOM $2 \mathrm{~N}$

FAIRFAX, VA 22037

$703849-3730$

PHILIP C OLSSON

PAR TNER

OLSSON \& FRANK

1029 VERMONT AVE NW SUITE 400

HASHINGTON. DC 20005 
GERALD H ONEILL

DISTRIC T MAINTENANCE ENGINEER

AMOCO OIL COMPANY

150 S WARNER RD SUITE 432

KING OF, PA 19406

$215254-6100$

ALFRED N ORMOND

PRE SIDENT

ORMOND INC

12020 EAST RIVERA ROAD

SANTE FE. CA 90670

$213945-1427$

GEORGE W OSOSKE

EXECUT I VE VICE PRES IDENT

CAL IFORN I A BREWERS ASSOCIAT ION

235 MONTGOMERY STRE ET H5O8

SAN FRANCISCO, CA 94104

$415421-7747$

JOSEPH W OVELLA

SERVICE MANAGER

G T MICHELLI CO INC

$P$ O $80 X 1045$

METAIRIE LA 70004

$504885-9710$

JERRY OVERTON

MANAGER OF QUAL ITY CONTROL

WALNUT GROVE PRODUC TS

SECOND AND LINN

$A T L A N T I C$
712 I IA

CHARLES K OVIAN

MGR NEIGHTS \& MEASURES DEPT

9 MONA VISTA AVE

RUTLAND: VT 05701

$802775-1571$

JIM C OWNBEY

VICE PRESIDENT \&RASWELL SCALE \& EQUIPMENT CO

P O BOX 5422

ASHEVILLE, NC 28813

$704274-3771$

PHILLIP A PAINCHAUD

CONSULTANT

METROLOGY EALLIED TECHNOLOGIES

$1110 \mathrm{~W}$ DOROTHY DR

BREA, CA 92621

$714529-6604$

CHARLES R PALMOUIST

GASOLINE SUPERVISOR

SOUTHLAND CORPORATION

8825 ROE AVE

PRAIRIE VILLAGE, KS 662072299

$913649-8220$

CLAUDE R PARENT

DIRECTOR-NATIONAL ACCOUNTS

GIL $8 A R C O$ INC

7 EASTWOOD DR

OR INDA. CA 94563

$415376-5697$

SHARON PARKER

OFF ICE MANAGER

INTERNATIONAL ROAD DYNAMICS

$211-3502$ TAYLOR ST E

SASK SASKATCHEWAN

RICHARD S PARRILL

DIRECTOR OF CUSTOMER SERVICES

HOLLY SUGAR CORPORATION

$P 080 \times 1052$

COLORADO SPRINGS, CO 80901

$303471-0123 \times 208$
EDWARD F PASCHAL

CIV IL ENGINEER

MISSOURI PACIFIC RAILROAD CO

RM $1211210 \mathrm{~N} 13 T H$ STREET

ST LOUIS MO 63103

$314622-2861$

NARENDRA S PATEL

BRIDGE \& STRUCTURE ENGINEER

GRAND TRUNK WESTERN RAILROAD

131 LAFAYETTE RM 903

DETROIT MI 48226

G IAN PATTERSON

P ENG

LIQUID TRANSFER SYS LTO

1325 SHAWSON DR/MISSISSAUGA

ONT ARIO L4W

RONALD A PATTERSON

PRESIDENT

PUMP PARTS INC

12427 GRANO RI VER

DETROIT. MI 48204

$313933-9500$

JOHN F PAUGSTAT

ENG INEER ING \& MFG CAM8RIDGE NCR CORPORATION

PO $80 X 728 / 800$ COCHRAN AVE

CAMBR IDGE. OH 437250523

$614439-0571$

DONALD L PAUL

VP OUAL ITY AND REGULATORY AFFR OSCAR MAYER FOODS CORPORATION PO BOX 7188

MAD ISON. WI 53707

$608241-6803$

JAMES E PEEPLES

DIRECTOR LEGISLATIVE AFFAIRS

INF ORMATION RESOURCES INC

499 S CAPITOL ST SUITE 406

HASHINGTON, DC 20003

$202554-0614$

H STEFFEN PEISER

638 8LOS SOM DR

RDCKVILLE. MO 20850

$301762-6860$

RO8ERTO PERAZA

GASOL INE CONSTRUCTION MANAGER SOUTHLAND CORPORATION

$P 0$ 80X 719

DALLAS. TX 75221

$214559-0850$

PETER R PER INO

PRESIDEN I

TRANSDUCERS INC

14030 BOLSA LANE

CERRITOS. CA 90701

$714739-1991$

W H PERRY

PRESIDENT

CARDINAL SCALE MFG CO

P 0 80X 151

WEBB CITY. MO 64870

$417673-4631$

ALBERT A PESENII

YICE PRESIDENT SALES

DUNCAN INDUSTRIES

1701 GOLF ROAD SUITE 1100

ROLLING MEADOWS. IL 60008

$312952-2990 \times 210$ 
ROBERT J PETERSEN

PROGRAM MANAGER

AMERICAN NATIONAL METRIC COUNC

1010 VERMONT AVE N W

AS SHINGTON, DC 20005

$202628-5757$

FREDERIC M PETLER

HEAD ADMINISTRATION

U S SUZUKI MOTOR CORPORATION

3251 EAST IMPERIAL HWY

BREA, CA 92621

$714996-7040 \times 278$

THOMAS JOSEPH PETRY

EXECUTI VE DIRECTOR

NATIONAL STANDARDS COMM.

PO BOX 282

NORTH RYDE.

RICHARD C PHELPS

DIRECTOR OF MANUFAC TUR ING

REX PLASTICS INCORPORATED

1200 UN ITY

THOMASVILLE: NC 27360

DA VID PHILLIPS

INSPECTOR

BURLING TON NORTHERN RAILROAD

1300 EAST H ST APT 29

MCCDOK NE 69001

RAMONA PHILLIPS

OUALITY CONTROL SUPERVISOR

MONTEREY MUSHROOMS

HWY 75 SOUTH P D BOX

MADISONVILLE, TX 77864

409 348-3511

RICHARD L PHILLIPS

MID MIS SOURI SCALE INC

RR 4 BOX $193 \mathrm{~F}$

SEOALIA. MO 65301

$816827-4571$

C THOMAS PICTON

SYSTEM SUPERVISOR SCALE INSPEC CONRAIL

6 PENN CENTER RODM 1634

PHILADELPHIA, PA 19104

$215977-1617$

HY PITT

PRESIDENT

PITT TRAINING ASSOCIATES

$6810 \mathrm{~N}$ NEIL PL

MIL WAUKEE: WI 53209

$414352-3277$

JARVIS J POCHE

ASST MATER IALS ENGI NEER

LOUISIANA DEPT OF TRANS E DEV

BATON ROUGE: LA 70804

504 $925-6722$

HENRY L POLITI

PETROL MGMT SYSTMS-USG 3

NCR

$1700 S$ PATTERSON BLVD

DAYTON, OH 45479

$314991-0273$

HARDY 8 POOLE

ASST DIR-GVT REL - REGULATORY

AMER IEXTILE MFGRS INSTITUTE

1101 CONNECTICUT AVE 300

WASHINGTON, DC 20036

$202862-0519$
VICTOR L PRESTON

BALANCE \& SCALE REPAIRMAN

PROCTER \& GAMBLE COMPANY

6083 CENTER HILL RD

CINC INNATI, OH 45224

$513659-7073$

RALPH PRETZER

SUPERVISOR CORPORATE PACKAGING DOW CORNING CORP

MAIL \#112 P O BOX 1592

MIDLAND, MI 48640

$517496-4000$

DONALDW PROTHEROE

PRODUCT ION MANAGER

MONTEREY MUSHROOMS-B TERRE

$P$ O BOX 250

BONNIE TERRE, MO 63628

$314358-3381$

JOHN PROVETERO

PLANNER

I BM

G68/B602 PO BOX 12195

RESEARCH TRIANGLE, NC 27709

$919836-5456$

RANDY PUGH

VICE PRESI DENT

CORRELLS SCALE SERVICE

RT 51450 NELSON RD

MOSES LAKE. WA 98837

$509765-7754$

JAMES $J$ QUANDT

SENIOR AUTOMOIIVE SPECIALIST

STANDARD DIL COMPANY (OHIO)

4850 E 49TH ST

CLEVELAND, OH 44125

$216575-4383$

CARL L QUINN

PRES IDENT

382 MARTIN AVE

SANTA CLARA. CA 95050

$408727-3611$

EDWARD A RACHINS

PRESIDENT

MUTUAL OIL CO INC

$P$ O BOX 486

BROCKTON, MA 02403

$617583-5777$

D HOWARD RANDALL JR

TECHNICAL PRODUCT MANAGER

ESSELIE METO INC

39 TEED OR

RANDULPH, MA 02368

$617986-6200 \times 37$

DIEDRICH RANDERS-PEHRSON

MECH ENG

VEGA VEKT $A / S$

$P$ D BOX $158 \mathrm{~N}-1360$

NESBRU

CHARLES RASCHER JR

RASCHER \& BETZOLD INC

5410 N DAMEN AVE

CHICAGO. IL 60625

$312275-7300$

$\checkmark I C$ RASHEED

EXECUTIVE DIRECTOR

SERVICE STATION DEALERS OF AME 400 NORTH CAPITOL ST SUITE 175 WASHINGTON, DC 20006

$202393-6868$ 
LARRY M RASMUSSEN

QUALITY ASSURANCE ENGI NEER

PETER ECKRICH AND SONS INC

$P$ O BOX 388

FORT WAYNE, IN 46801

$219481-2034$

IHOMAS L RAWLINGS

SENIOR PACKAGE SPECIAL IST

BLACK \& DECKER INC

6 ARMSTRONG ROAD

SHELTON, C 106484

$203926-3364$

8 D RAY

VICE PRESIDENT ENGINEER ING

VINCE HAGAN CO

PO BOX 225141

DALLAS. TX 75265

$214339-7194$

STEPHEN RAYNER

STAFF OFFICER

USDA AGRICULTURAL MARKETING SE

14 TH $\&$ INDEPENDENCE RM OTO9S

HASHING TON, DC 20250

$202447-5021$

GORDON F REED

PRESIDENT

REED TRUCKING

GO MELROSE ROAD

GRAND ISLAND. NY 14072

ARTHUR L REENSTRA

DIR OF ENGINEERING/MANUF DIV

FRANKLI N ELECTRIC CO PACK/WHG

PO BOX 666

LEVITTOWN: PA 19058

$215295-2000 \times 63$

2 REFEROWSKI

ASSISTANT DIRECTOR

INT BUREAU OF LEGAL METROLOGY

11 RUE TURGOT

75009 PARIS

MAR SHALL D REHM

FOSSIL FUELS ENG INEER

PA POWER \& LIGHT CO

$2 \mathrm{~N} 9 \mathrm{TH}$ ST

ALLENTOWN, PA 18101

$215821-4714$

JAMES REICHE

TECHNICAL PROJECTS MANAGER

REFINED SUGARS INC

1 FEDERAL STREET

YONKERS. NY 10702

$914963-2400$

FRED E REIKOWSKY

QUALITY CONTROL MANAGER

LUFKIN DIV COOPER IND

$80 \times 728$

APEX. NC 27502

$919362-7511 \times 636$

ROBERT A REINFRIED

TECHNICAL ASSISTANT

SCALE MANUFACTURERS ASSN

152 ROLLINS AVE

ROCKVILLE. MD 20852

$301984-9080$

ROBERT F REISER JR

OPERATI ONS MANAGER

CONDON OIL COMPANY

126 E JACKSON ST

RI PON, WI 54971

$41474 B-3186 \times 41$
LUKE RENZI

PROJECT LEADER

AGREX INC

PIER A BER IH 211 AGREX BLK TER LONG BEACH. CA 90802

$213432-1864$

STEVE J REYNOLDS

ELECTR ICAL ENG INEER

EX-CELLO MATER IALS HANDLING CO PO BOX 6549 JOHNSON PKWY-AMES ST PAUL. MN 55106

$612776-8501$

AUSTIN T RHOADS

NORTHEAST ICE CREAM ASSN

$P 0$ BOX 0510

FUL TON, MD 20759

$301953-9117$

DONALD P RICE

ASSI DIR QUALITY ASSURANCE

STROH BREWING CO

100 RIVER PLACE

DETROIT. MI 48207

$313446-2157$

ROBERT S RICHARDS

BALANCE ANO SCALE REPAIRMAN

PROCTER E GAMBLE CO

6083 CENTER HILL RD

CINCINNATI OH 45224

$513659-7073$

ERVIN G RICHARDSON

CORPORATE ENG GOVT STANDARDS

REYNOLDS METALS COMPANY

6601 W BROAD ST

RICHMOND, VA 23261

$804281-2276$

DAVID M RICHMAN

DIRECTOR OF QUALITY ASSURANCE

GIANT FOOD INC

PO $80 \times 1804$

WASHINGTON: DC 20013

$301341-4134$

JOHN R RIDDELL

MANAGER TRAFFIC

COLUMBIAN CHEMICALS COMPANY

1600 PARKWOOD CIRCLE NW RM 400 ATLANTA. GA 30339

$404951-5700$

GEORGE RIEMANN

TECHNICAL DIRECTOR

FUEL MERCHANTS ASSOCIATION NJ

66 MORRIS AVE

SPR INGFIELD. NJ 07081

$201379-1100$

SIERT F RIEPMA

PRESIDENT

NATIONAL ASSN OF MARGARINE MFR 1625 EYE STREEI NH SUITE 1024 A WASHINGTON, DC 20006

$202785-3232$

JACK A RISER

MANAGER LABORATORY SERVICES

KEEBLER COMPANY

ONE HOLLOW IREE LANE

ELMHURST: IL 60126

$312833-2900$

JOHN R RIZZO

VICE PRESIDENT

BUFFALO SCALE \& SUPPLY COMPANY

2609 CHART IERS AVE

PITTSBURGH. PA 15204

$412 \quad 331-3335$ 
DAVID C ROBBINS

PRESIDENT

COMMERC IAL SCALE CO INC

INDIAN DRCHARD, HA 01151

$413543-2290$

DHIGHT G ROBERTSON

SENIOR ENG INEER - PER FOR MANCE SOUTHWESTERN PUBLIC SERVICE CO 6TH \& TYLER

AMAR ILLLDIX 79170
$806378-2722$

$J O H N$ J ROBINSON

SR ASSI STANT VICE PRES IDENT ASSOCIATION OF AMERICAN RR

$50 \mathrm{~F}$ ST NW

HASHINGTON, DC 20001

$202293-4144$

MARK RODGERS

PROJECT ENGINEER

BERKEL INC

ONE BERKEL DR

LAPORTE IN 46350

$219326-7000$

STU ROEMKE

KROGER COMPANY

2 CAMPBELL DR

HI GHLAND HEI GHT S, KY 41076

$607572-2223$

ALAN F ROGERS

MANAGER OUALITY ASSURANCE

UNION CARBIDE H $\&$ A DIVISION

88 LONG HILL STREET

EAST HARTFORD. CT 06108

$203728-6086 \times 350$

GLENDA ROGERS

OUALITY ASSURANCE MANAGER

SOUTHER N TEA COMP ANY

1267 COBB INDUSTRIAL DRIVE

MAR IETTA GA 30066

$404428-5555$

OL IVER D RDHRBACK

DIVISION 8416

SANDIA NATIONAL LABORATORIES

LI VERMORE, CA 94550

$415422-3146$

DANIEL ROMAN

QUALITY CONTROL ENG INEER

SWEDA INTERNATIONAL INC

34 MAPLE AVE P

PINEBROOK. NJ 07058

$201575-8100$

JOSEPH P ROMANO

HTS $E$ MEAS INSPECTOR III

NOXELL CORP

11050 YORK RD

HUNT VALLEY: MD 21030

$301628-4435$

TERRY ROSFELDER

SUN REF INING $\varepsilon$ MARKEIING

1801 MARKET ST

PHILADELPHIA, PA 19103

$215977-6502$

ROBERT ROSS

FLUID MEASUREMENT SPECIALIST

P O BOX 2040

TULSA, OK 74102

$918599-4200$
HENRI G ROUQUAIROL

DIRECTOR OF APPROVALS

MOREAU S A

106 BIS AVENUE BEAUREPAIRE

94100 SAINT-MAUR

$0113318861 \times 283$

ED RUGENSTEIN

HHY ENGR HCT-1

FEDERAL HIGHWAYY ADH DOT

4007 TH ST SH

HASHINGTON: DC 20590
$202426-6282$

BILL RUSSELL

PRESIDENT

BILL RUSSELL OIL CO INC

215 EAST 9 TH

RECTOR: AR 72461

$501595-2427$

JOE E RUZICKA

PACKAGING SYSTEMS ENGINEER

IMPER IAL SUGAR CO

SUGAR LAND. TX 77478

DONALD P RYAN

CHIEF DR AF TSMAN

STANLEY TOOLS-DV STANLEY WORKS

600 MYRTLE ST

NEW BRITAIN. CT 06050

$203225-5111 \times 453$

PHIL RYBACKI

NATIONAL RETAIL MKTG MGR

SCHWANS SALES ENTERPRISES

MAR SHALL MN 56258

$507532=3274$

GER ARD SAB IRON

DIR RESEARCH \& DEVELOPMENT

ESSELTE MOREAU

94100 SAINT

DAMON H SAMPSON

WEI GHMASTER

TRISTATE GRAIN INSPECTION SVC

3906 RIVER ROAD

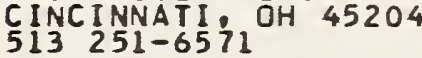

PAUL SAMSING

CONSULTANT

PAUL SAMSING CONSULTANT

245 NORTH 16 ST

MONTEBELLO CA 90640

$213944-6111 \times 323$

MICHAEL SANNA

MANAGER OUALITY MANAGEMENT

BRADY ENTERPRI SES INC

167 MOORE ROAD

EAST WEYMOUTH. MA 02189

$617337-5000 \times 21$

RICHARD D SANNE

ACT ING DIRECTOR

NEBRASKA GRAIN \& FEED DEALERS

1620 H STREET FIRST FLR SDUTH

LINCOLN, NE 685082531

$402476-6174$

$R$ ICHARD $R$ SAUNDERS

SEC \& TREAS

$R$ H MCCOLLUM CO INC

107 MEADOWVIEW RD BOX 20145

GREENSBORO. NC 27420

919 272-6191 
DAVID J SAVAGE

DIRECIOR OF QUALITY AS SURANCE DRACKET T COMPANY

5020 SPRING GROVE AVE

CINCINNATI OH 45232

$513632-1406$

DENNIS SCHAFFER

SCALE SER ICE COORD INATOR

19250 VAN NESS AVE

TORR ANCE: CA 90501

$213320-8900$

SHELDON SCHAFFER

PRESIDENT

WELD-IT COMPANY

4477 SHEILA ST

LOS ANGELES, CA 90023

$213263-7524$

MICHAEL J SCHAI 8LE

SPECIAL REPRESENTATIVE

MARATON OIL CO

539 MAIN ST

FINDLAY, OH 45840

$419422-2121$

MIKE R SCHEOWIN

VICE PRESIDENT

AB SCO SCALE CORP

3408 INDIANOLA AVE

COLUMBUS: $\mathrm{OH} 43214$

$614268-2626$

GROVER L SCHENCK II I

SUPT SCALES E HIGHWAY EQUIP

SOUTHERN RAILWAY SY STEM

99 SPRING ST SW

ATLANTA. GA 30303

404 529-1391

RICHARD H SCHIEBER

SENIOR TECHNICAL REPR

AMERICAN METER CO

13500 PHILMONT AVE

PHILADELPHIA. PA 19116

$215673-2100$

JEROME R SCHINOLER

ATTORNEY

8ORDEN INC LAW DEPT BB-27

$P$ O BOX 2478

COLUMBUS, OH 43216

$614225-4336$

\section{RALPH S SCHLETIY}

PETROLEUM EQUIPMENT

CENEX

BOX 64089

ST PAUL. MN 55164

$612451-5348$

DIETER F SCHLUTER

SARTORIUS BALANCES/BRINKMANN

CANTIAGUE RD

WESTBURY, NY 11590
$516334-7500 \times 230^{\circ}$

MYRON A SCHMUTZER

VICE PRES QUALIYY ASSURANCE

600 YORK ST

ELI I ZABE TH. NJ 07207

$201527-3422$

MILES A SCHNEIDER

PRESIDENT AND GENERAL MANAGER

SCHNEIDER SYSTEMS

621 WEA VER ST

FAIRFIELD, OH 45014
HAR VEY SCHOCK JR

CONSULTANT

PRODUCT AS SURANCES

30 OAK RIDGE DR

HADDONFIELD: NJ 08033

$609428-7236$

JAMES A SCHRACK

PRESIDENT

EASTERN HEIGHING SYSTEMS INC 8 ATH PA 18014

$215759-2704$

ROBERT W SCHRADER

MGR LAB \& TECH SER V

ROSS IND DEPT OF CARGILL INC P 0 BOX 2696

HICHITA. KS 67201

$316267-6281 \times 250$

A SCHR AHM

AMOCO OIL CO

AMOCO OIL COMPANY

AC 1444A/PO BOX 5910-A

CHICAGO. IL 60680

HEL VIN H SCHROEDER

MANAGER REGULATORY 6 SAF SERV SUBURBAN PROPANE GAS CORP

$P$ BOX 206

WHIPPANY. NJ 08807

$201887-5300$

ARL IS SCHULTE

TRAFFIC MANAGER

COASTAL STATES ENERGY CO

NINE GREENWAY PLALA

HOUSTON. TX 77046

$713877-1400$

HAROLD L SCHULTZ

SUPERVISOR OF WEIGHTS

ENID BOARD OF TRADE

PO BOX 17472309 NORIH 1OTH

ENID. OK 73701

405 233-1528

JOHN H SCHULTZ

SALES MANAGER

RIEDE SYSTEMS INC

8375 SUNSET RD

MINNEAPOLIS. MN 55432

$612784-8020$

H E SCHUMACHER
OIRECTOR SALES \& SERVICE

KERR MC GEE COAL CORPORATION

KERR MC GEE CTR PO BOX 25861

OKL AHOMA CITY. OK 73125

405 270-3735

RON G SCHUMANN

MANAGER MAINIENANCE

SPEEDWAY PETROLEUM

2121 DIRECIORS ROW

INDI ANAPOL IS. IN 46241

$317243-7500 \times 224$

808 SCHWAR 8 ERG

BUREAU CHIEF

ILLINOIS OEPT OF AGRICULTURE

801 EAST SANGAMON AVENUE

SPR INGF IELD, IL 62706

$217782-3817$

JAMES C SCHWARTZ

PROJECT LEADER

UNC NAVAL PRODUCTS

67 SANDY DESER I RD

UNCASVILLE, CT 06382

$203848-15 i 1 \times 2482$ 
Y W SCOTT

KELLOGG COMPANY

BATTLE CREEK, MI 49016

TIMOTHY L SCRIBNER II

PROJECT ENGINEER

OWENS CORNING FIBERGLAS

300 SUN SHINE ROAD

KANSAS CITY: KS 66102

$913281-9528$

TOM SCR I VENER

VICE PRESIDENT

FRAZIER PRECISION INSTRUMENT

16761 OAKMONT AVE

GAI THER SBURG, MD 20877

RICHARD C SEALE

PRESIDENT

KAMLAR CORP

105 KAMLAR DRIVE

ROCKY MOUNT: NC 27801

$919443-2576$

$S$ O SEILING

PROJECT ENG INEER

AMF UNION MACHINERY DIVISION

P O BOX 9168

RI CHMOND, VA 23227

$804355-7961$

RICHARD L SEITZ

MGR MECH PRODUCT ENGR

VEEDER ROOT CO

HATFORD: CT 06102

$203527-7201 \times 430$

JAMES A SERVIN

DIR CON SUMER AFFAIRS DIVISION DEPT OF PUBLIC \& CONS AFFAIRS 25 GRENFELL ST GRENFELL CENTRE ADELAIOE SA $2283211 \quad \times 247$

T SESHAGIRI

PARTNER

GIRI BROTHERS

51 RAJAJI SALAI MADRAS 600001 MADRAS TAMIL

CHARLES C SHANNON

OPERATIONS MANAGER

HEENAN OIL CO INC

$P$ B BOX 67

LEVITTOWN: PA 19059

MAX SHAW

TECHNICAL DIRECTOR

FR I GO CHEESE CORPORATION

$P$ O BOX 158

LENA. WI 54139

$414829-5251$

BARRY F SHEA

ASSOC LEGAL COUNSEL/ASST SECY WEST PO INT-PEPPERELL INC

PO BOX 71

WEST POINT, GA 31833

$205756-7111 \times 3163$

JACK SHEEHAN

MANAGER

HOBART CORP

28 BY PASS BOX 957

MANCHESTER, NH 03105

$603623-3622$
DAV ID $B$ SHEPARO

VICE PRESIDENT MANUFACTURING NAT IONAL CONTROLS INC

2320 AIRPORT BLVD PO BOX 1501

SANTA ROSA. CA 95402

$707527-5555 \times 131$

DARYL C SHIPMAN

SALES ENGINEER

NEP TUNE MEASUREMENT CO

EMERALD ROAD P D BOX 792

GREENWOOD, SC 29646

$803223-1212$

GLENN C SHIROMA

GENERAL PARTNER

SCALE SYSTEMS HAWAII

$P$ O BOX 1598

HILO, HI 96720

$808935-1344$

RICHARD M SHIRVEN

SER VICE MANAGER

SNAP-ON TOOLS CORP

$280180 T H$ ST

KENOSHA, WI 53140

$414656-5391$

ROGER E SHOEMAKER

PARTNER

WEBTER SCALE SALES AND SERVICE PO BOX 127

WEBTER, SD 57274

$605345-3881$

ALBERT J SILHAYY

HEIGHT SUPERVI SOR

FORT WOR TH GRA IN EXCHANGE

2707 DECATUR AVE PO BOX 4422

FORT WORTH, TX 76106

$817626-1071$

PAUL SIMITOSKI

LABORAIORY DIRECTOR

PENN CHAMP INC

$14 T H$ E RAILROAD ST

EAST BUTLER, PA 16029

$412287-8771 \times 33$

MINDAUGAS A SIMKUS

MANAGER OF PROCESS ENGINEER ING THE OUAKER OATS COMPANY

345 MERCHANDISE MART

CHICAGO, IL 60654

$816279-1651 \times 326$

FRANK $X$ SINGLETON

PRESIDENT

MARYLAND PUMP TANK \& ELECTRIC

2512 ERICK STREET

BAL I I MORE, MD 21230

301 837-0770

MARVIN SIROTA

CORPORATE DIRECTOR MANUFACTURE HELENE CURTIS INDUSTRIES INC

4401 H NORTH AVE

CHICAGO. IL 60639

$312292-2121$

HARTVIG W SKAUGE

AMERICAN CRYSTAL SUGAR CO

NOR TH IITH PO BOX 1037

MOORHEAD, MN 56560

$218233-1561$

JEFFREY A SLACK

PRODUCTI ON MANAGER

AGWAY INC MOTOR TRANSPORTATION

44 R I VER ST

CORTLAND. NY 13045

$607753-3091$ 
KENNETH SLENTZ SR

QUALITY CONTROL FLD OPR MGR

EASTERN MILK PRODUCERS

6567 KINNE RD

SYRACUSE, NY 13214

$315446-0730 \times 36$

DOUGLAS M SMITH

MANAGER

ABBOTT LABORATORIES

1400 SHER IDAN RD / 736-M4

NOR TH CHICAGO. IL 60064

$312937-4929$

GAYLORD SMITH

QUALITY CONTROL ASSURANCE DIR NEDCO

44 UNION ST

FUL TONVILLE, NY 12072

JAMES L SMITH

PRESIDENT

SMITHS SCALE SERVICE INC

2509 8TH AVE

COUNC IL BLUFFS, IA 51501

$712322-8671$

LEE SMITH

NEW PRODUCTS MANAGER

MICRO MOTION INC

7070 WI NCHESTER CIR

BOULDER . CO 80301

$303530-0530$

MAR ILYN SMITH

TREASURER

CAP ITOL SCALE CO INC

927 SW WASHINGTON ST

PEORIA. IL 61602

309676-0865

MICHAEL L SMITH

SALES MANAGER

EAGLE MICROSYSTEMS

RT $100-R D$ H 2

CHESTER SPRINGS, PA 19425

$215458-5778$

WILLIAM A SMITH

PROCTER \& GAMBLE COMPANY

11520 REED HARTMAN HW/PO 41520

CINNCINNAT I OH 45241

$513763-6075$

CARL A SMITH JR

OIRECTOR PRODUCT SAFETY - AGRI

PILLSBURY CO

PILLSBURY CENTER

MINNEAPOLIS: MN 55402

$612330-5165$

CARL A SMITH JR

OIRECTOR QA TECHNICAL SERVICES

PILLSBURY COMPANY-MS 9441

3 II SEC OND STREET S E

MINNEAPOLIS, MN 55414

$612330-4477$

\section{JACK T SNADER}

EXECUTIVE VICE PRESIDENT

SEEDBURO EQUIP CO

$1022 W$ JACKSON BLVD

$312738 \div 3700$

VINCENT SNYDER JR

STATE REGULATORY SPECIALIST

O M SCOTT \& SONS COMPANY

MARYVILLE, OH 43041
$513644-0011 \times 2497$
LOUIS F SOKOL

PRESIDENT EMER I TUS

U S METRIC ASSOCIATION

SUGARLDAF STAR RT

BOULDER, CO 80302

$303442-3342$

LARRY D SOLE

DIRECTOR QUALIIY ASSURANCE

TEXIZE DIV OF DOH CHEMICAL CO PO BOX 368

GREENVILLE, SC 29602

ARTHUR SOLIS

WEIGHT SYSTEMS SPECIALIST

DOW CHEMICAL USA

PO BOX 1398 LOVER IDGE ROAD

PITTSBURG. CA 94565

$415432-5006$

JOHN E SOLTES

DIREC TOR OF PACKAG ING

HYPONEX CORPORATION

4501 CIRCLE 75 PKWY STE 82250

ATLANTA, GA 30339

$404955-5910$

EDWARD H SONN

VP PRODUCT MARKET ING

DATACHECKER/DTS

124 ACTON ST

MAYNARD. MA 01754

$617897-3221 \times 2400$

TAYLOR SOPER

PRESIDENT

FAIRBANKS WEIGHING DIVISION

COLT INDUSTRIES

ST JOHNSBURY, VT 05819

$802748-5111 \times 300$

RICHARD SOUTHERS

6020 CHATSWORTH LANE

BETHESDA. MD 20814

$301530-8327$

WILLIAM J SPAIN

DIRECTOR REGUL AFFRS ENERGY MG

DEL MONIE CORPORATION

PO BOX 3575

SAN FRANCISCO, CA 94119

$415 \quad 442-4610$

R L SPARKS

GENERAL SUPERINTENDENT ISO

MONSANTO POLYMER PRODUC TS CO

NITRO. WV 25143

$304755-334$

JOHN F SPEER JR

PRESIDENI

MILK \& ICE CREAM ASSOCIATIONS

$88816 \mathrm{TH}$ STREET NH

WASHINGTON. DC 20006

$202296-4250$

SAMUEL K SPIGLER SR

PRESIDEN I

SAMUEL $K$ SPIGLER COMPANY INC

$P 0$ BOX 360

FUNKSTOHN, MD 21734

$301739-4462$

HAROLD SPILKER

MEAT MERCHANDI SER

BALLS SUPER FOODS INC

5300 SPEAKER ROAD

KANSAS CITY. KS 66106

$913321-4223 \times 50$ 
OR VILLE E SPILKER

SPIKES FOOD \& MEAT EQUIPMENT

7629 ENGLEHOOD

RAYTOWN, MO 64138

8 !6 $358-4600$

JAHES H SPIROS

PRESIDENT

SUPERIOR EQUIPHENT COMPANY INC 3283 IVANHOE AVE

SAINT LOUIS, MO 63139

$314644-6000$

WILLIAM D SPOHN

DIRECTOR MFG SERVICES

FRANKLIN CHEHICAL INDUSTRIES

2020 BRUCK ST

COLUMBUS, OH 43207

LEROY A SPRINGMAN

MANAGER QUALITY CONTROL

ELI LILLY AND CO STREET

307 EAST MCCARTY STREE

$317261-4686$

LEON L ST LOUIS

MAINTEN ANCE SUPERVISOR

HEBBER OIL COMPANY

700 MAIN ST

BANGOR, ME 04401

$207942-5501 \times 276$

THOMAS STABLER

MANAGER - WTS AND MEAS

TOLEDO SCALE

$P$ O BOX 658

HORTHINGTON, OH 43085

$614438-4548$

MARLAN D STAHL

NANAGER

3737 E BROADWAY

PHOENIX, AZ 85040

$602268-1381$

JOEL ST ANGELAND

MANAGER CORPORATE QUALITY ASSU LAND D LAKES INC

P O BOX 116

MINNEAPOLIS, MN 55440

$612481-2281 \times 379$

JERRY STANKORB

GENERAL CHEMIST

AMERICAN CRYSTAL SUGAR CO

101 NOR TH THIRD ST

MOORHEAD, MN 56560

$218236-4400$

HILLIAM Y STANSKY

Q A INSPECTION MANAGER

MENNEN COMPANY

MORRISTOWN, NJ 07960

$201631-9095$

FRED J STAUDINGER

BUSINESS SEGMENT MANAGER

PITNEY BOHES INC MSD

WALTER H WHEELER JR DRIVE

STAMFORD. CT 06926

$203356-7019 \times 404$

\section{KENNETH WTEEN}

PRESIDENT

R H ALE XANDER CO

3001 E 12TH ST

LOS ANGELES, CA 90023

$213264-1141$
RAFAEL STEINBERG

PRESIDENTE SISTEMA

INTERAMER ICAND DE METROLOGIA

SIM-INTI CC 157

1650 SAN 7525213

VIC HO STEINHOFFER SR

OWNER

MICHIANA SCALE COHPANY

1524 H139

BENTON HARBOR, MI 49022

$616927-1471$

WILLIAM C STEHART

ENGINEER ING SERVICES COORD

EXXON COMPANY USA 38719

P $\mathrm{BOX} 4388$

HOUSTON, TX 77210

$713656-6170$

C A STOFFERS

MGR FOOD TECHNOLOGY DIVN

SAFEWAY STORES INC

$430-A$ JACK SON ST

OAKLAND, CA 94660

$415891-3250$

ROBERT STOKES MAR IND GVT E REG ACTIVITIES PO BOX 599

CINCINNAII 513 OH 45201

JACK STOUGH

EXECUTIVE DIRECTOR

INDIANA OIL MARKETERS ASSN

8780 PURDUE ROAO

INDIANAPOL IS. IN 46268

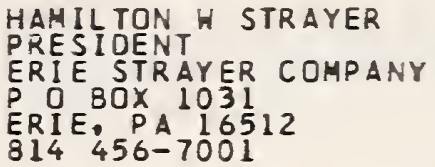

DAVID L STRONG

REG IONAL MANAGER

TOTAL PETROLEUH INC

1019 N BURL ING TON

N KANSAS, MO 64116

$816421-2200$

RANDALL L STRUBHAR

MANAGER OUALITY CONTROL

LEHN \& FINK PROO/STERLING DRUG 316 N LIHIT ST

LINCOLN, IL 62656

FRED L STULTZ

GEN MGR

ACME SCALE CO

1801 ADAMS AVE

SAN LEANDRO, CA 94577

$415638-5040$

MAX S STURGES

MGR OUALITY AS SURANCE E FLAVOR POPSICLE INDUSTRIES INC

110 ROUTE 4 P O BOX 200

ENGLE WOODDNJ 07631

JAMES D SUGGS

SCALE SERVICE

XANSAS CITY, MO 64117

$816454-9509$ 
EARL R SULLIVAN

DEPUTY DIRECTOR

ASTM

1916 RACE STREET

PHILADELPHIA? PA 19103

$215 \quad 299-5514$

EDWARD H SULLIVAN

OUALITY ASSURANCE MANAGER

LEVER BROTHERS COMPANY

5300 HOLABIRD AVE

BAL TIMORE, MD 21224

$301342-2100 \times 322$

JOHN H SULLIVAN

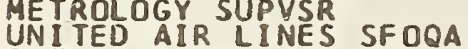

INTL AI RPORT

SAN FRANCISCO, CA 94128

$415876-4780$

T S SUN

MGR QUAL CONTROL/AS SURANCE

WALGREEN LABS INC

3308 CDVINGTON ROAD

KALAMAZ OO. MI 49002

$616349-1096$

$J$ M SURACI

MANAGER CAL IBRATION LABORATORY LOCKHEED MISSILES \& SPACE CO

PO BOX 6429

BREMERTON, WA 98315

206 396-6772

GRENVILLE G SUTCLIFFE

HUSKY CORPORATI ON

I DAILEY INDUSTRIAL PARK

PACIFIC, MO 63069

$314257-3073$

SE IJI SUZUKI

TOKYO ELECTRIC USA INC

19250 VAN NESS AVE

TORRENCE CA 90501

213 320-8900

PHILIP E SWANSON

SR ENGI NEER

SMITH METER INC

1602 WAGNER AVE

ERIE PA 16514

$814899-0661 \times 268$

URBAN J SWEENEY

CHIEF LIBRAR I AN

GENERAL DYNAMICS CONVAIR DIV

$P$ O BOX 85386

SAN DIEGO CA 92138

$619573-4876 \times 1073$

PHIL SHEET

QUALI TY ASSURANCE TECHNOLOGIST

PLANTERS \& LIFESAVERS

200 JOHNSON AVENUE

SUFFOLK, VA 23434

$804934-6436$

BRYAN L SWINNEY

MARKETING, SYSTEMS ENGINEER

ENG INEE RED SYSTEMS INC

2001 WE ST CAMPUS DR

TEMPE $A Z \quad 85282$

$602438-1362$

AUGUSTUS E SYERS

INSTRUMENT SUPERVISOR

E R SOUIBB \& SONS INC

NEH BRUNSWICK. N J 08903
HUGH W SYMONS

VICE PRESIDENT RET SERVICES

AMERICAN FROZEN FOOD INSTITUTE

1700 OLD MEADOW RD

MCLEAN, VA 22102

$703821-0770$

LAWRENCE K TABER

CAL IFORN IA LEAGUE FOOD PROCESS

1007 L S

SACRAMENTO CA 95814

$916444-9260$

YUK IHIRO TAKADO

PRODUCT MANAGER

TOKYO ELECTRIC USA INC

19250 VAN NESS AVENUE

TORRENCE, CA 90501

$213956-2614$

F TAMBL INGSON

ANDERSON PEAT-ORGANIC COMPOST 2013 S ANTHONY BLVD

FT WAYNE, IN 46803

ROBERT B TANNER

SECRETARY

TANNER OIL CO

620 S FRONT ST

I RONTON OH 45638

$614532-9145$

FAROOK TAUF IO

VICE PRESIDENT QUALITY ASSUR

PRINCE FOODS INC

PRINCE AVENUE

LOHELL. MA 01853

$617458-4111 \times 121$

C I TAVERNER

PRINCIPAL TECH REPRESENTATIVE

PHILLIPS PETROLEUM COMPANY

11 D- 4 PHILLIPS BLDG

BAR TLESVILLE, OK 74004

$918661-4944$

BEN 2 TAYLOR

TECHNICAL DIRECTOR

PETERSON PURITAN INC

HEGELER LN

DANVILLE IL 61832

$217442-1400 \times 0315$

RALPH TAYLOR

SER VICE SUPERVISOR

HAMHEL SCALE UF KC INC

8421 NIEHAN ROAD

LENEXA. KS 66214

$913492-1807$

RICHARD N TENNILLE MANAGER TECHNICAL DEVELOPMENT CONTINENTAL PIPE LINE COMPANY $P$ BOX 2197

HOUSTON, TX 77252

$713293-1776$

HAMILTON I TEHKSBURY

SOUTH SHORE SCALE CO

81 ELM ST

COHASSETT. MA 02025

THE LIBRAR IAN

DEPARTMENT OF LABOUR

PRIVATE BAG

WELLINGTON 
B JORN THELEMARK

GEN MGR-PRESSDOCTORESENSOR DIV ASEA INC

ONE ODELL TERRACE

YONKERS. NY 10701

$914428-6000$

ELLEN THOMAS

MANAGER REGULATORY COMPLIANCE KRAFT INC

KRAFT COURT

GLENVIEW: IL 60025

RONALO A THOMAS

PRESIDENT

UNITED SCALE CORP INC

P BOX 376 , VA 24609

CEDAR BLUFF, VA 24609
$703963-0343$

RON THOMLEY

ASSISTANT MANAGER

SER VICE EQUIPMENT COMPANY INC 502 EAST MAIN STREET

DOTHAN, AL 36301

$205793-6676$

EARL A THOMPSON

MAR KETI NG DEPARTMENT

AMER ICAN PETROLEUH INSTITUTE

1220 L ST NW

ASHING TON, DC 20005

202682-8230

MERRILL S THOMPSON

VICE PRESIDENT \& PARTNER

CHADHELL AND KAYSER

P O BOX 8500

BRIOGETON IN 47836

$317548-2202$

PARKER A THOMPSON

MARKEI I NG MANAGER

STANLEY TOOLS

600 MYRTLE ST

NEW BRITAIN, CT 06050

203 225-511

RICHARD THOMPSON

GENERAL MANAGER-SECRETARY

IILIAM PIKE COMPANY

7741 DIX AVE

DETROIT, MI 48209

$313849-1300$

HILLIAM G THOMPSON

UP

CALLIS-THOMPSON INC

PO BOX 177

HARR ING TON, DE 19952

$302398-3253$

JOHN M THOMPSON JR

GENERATION ENG HEALTH PHYSICS

NMPC -NMP NUC STAT UNIT 2

8223 PERRUG IA LANE

CLAY, NY 1304

$315425-2525$

PAUL C THORPE

OIRECTOR OF COMPLIANCE

E $E$ J GALLO WINERY

PO BOX 1130

MODESTO CA 95353

$209521-3490 \times 3490$

JOHN F THUER MER

ASSOC DIR OA E COMPLIANCE-FOOD BORDEN INC

COLUMBUS, OH 43229
MERRILL E THURMAN

SCALE MAINTENANCE ENGINEER

PRECISION SCALE

$720 \mathrm{~N}$ ELWOOD

SPRINGFIELD. MO 65802
$417831-0995$

HOWARD B TISCH

PARTNER

MEISSNER TISCH \& KLEINBERG

275 MADI SON AVE

NEW YORK, NY 10016

JOHN TITCOMB

GENERAL MANAGER

MOULDEN SUPPLY CO

3600 HIGHWAY 80 WEST BOX 1247

JACKSON: MS 39205

$601922-4611$

WAL TER TKACHUK

SHELL OIL COMPANY

TWO SHELL PLAZA

777 HALKER ST/TSP ROOM 1142

HOUSTON, TX 77001

$713241-0502$

DARYL E TONINI

TECHNICAL DIRECTOR

SCALE HANUFACTURERS ASSN

6842 ELM STREET SUITE 102

MCLEAN, VA 22101

$301984-9080$

TOM TOPALIS

MGR OUAL ASSURANCE-COMPLIANCE

THE QUAKER OATS COMPANY

617 WEST MAIN ST

BARRINGTON. IL 60010

$312381-1980 \times 2064$

MAT TORMEY

MARKETING MGR COOLING/ENERGY

DELAVAL AGRICULTURE DIVISION

11 OO NORTH CONGRESS AVE

KANSAS CITY. MO 64153

$816891-7700 \times 484$

ANABEL TORRES

GENERAL MANAGER

NATIONAL STANDARDS OF PR

O BOX AC CAPARRA STATION

SAN JUAN,PR 00922

$809751-7188$

RICHARD P TRASK

WTS $E$ MEASURES LIAISON

KOPPENS / SCHLUMBERGER

181 STETSON RD

NORWELL. MA 02061

$617849-1368$

STEVE C TROUT

OWENS-ILLINOIS INC GCD PLT

901 N SHABBONA ST

STREATOR. IL 61364

ALTON C TRUBEY

QUALITY CONTROL MGR FERT PROD

AGWAY INC

978 LOUCKS MILL RD

YORK. PA 17402

$717846-1286$

WILLIAM E TRUMBO

CHIEF ENG I NEER

BANKHEAD ENTERPRISES INC

PO BOX 93006 MARTECH STATION

ATLANTA. GA 30318

$404894-7911$ 
GEORGE P TSIARTZAZIS

ME TRICATION OFFICER

WE IGHTS AND MEASURES SERVICE

MIN OF COMM \& INDUSTRY

NICOSIA

$4034413 \times 24$

RICHARD TUCKER

ASST MANAGER CUSTOMER SERVICE TOKHEIM CORPORATION

P 0 80X 360

FT WAYNE IN 46801

$219423-2552$

JAMES $H$ TURNER

TURNER SCALE SERVICE

581-8 GEORGE TODD DR

MONTGOMERY AL 36117

$205271-3232$

WILLIAM P TWIGG

SEN IOR ENG INEER

WESTING HOUS AESD

PO BOX 746 MAIL STOP 550

BALTIMORE, MD 21203

$301765-3606$

TOM A ULICNY

SCALE OPERATIONS MANAGER

23640 RESEARCH OR

FARMING TON HILLS MI 48024

BARBARA $S$ UMBENHAUER

TECHNICAL WRITER

PENNSYL VANIA SCALE COMPANY

21 GRAYBILL RD

LEOLA $71765-2653 \times 553$

DAN UNGAR

CONSULTANT

UNGAR CONSULTATION SERVICES

30 LAFA YETTE DR

WDODMERE NY 11598
$516374-5690$

HOWARD UPTON

EXEC VICE PRES

PO BOX 2380

IULSA $0 K 74101$
$918743-9941$

WICK WAN EYCK

VICE PRESIDENT ENGINEER ING

GARNAC GRAIN CO INC

8000 WE ST 11 OTH STREET

OVERLAND PARK, KS 66210

$913661-6100$

CHARLES L VAN INWAGEN

SENIOR STAFF ENGINEER

SHELL OIL COMPANY

1200 MI LAM PO BOX 3105

HOUSTON. TX 772533105

713 241-1778

CHR ISTOPHER VAN METRE

TECHNICAL SUPPORT

TOKHEIM FCSI

600 CENTURY PLAZA

HOUSTON IX 77073

$713821-9865$

JOHN M VAN PERNIS

VICE-PRES MACHINE DIV

BLACK PRODUCTS COMPANY

13513 S CALUMET AVE

$312468-97006027$
DON $L$ VANDE BERG

OWNER

VANDE BERG SCALES

770 TTH ST NH

SIOUX CENTER. IA 51250

$712722-1181$

GEORGE W VANDYKE

OWNER

VAN DYKE SCALE CO

2825 RAYTOWN RD

KANSAS CITY HO 64128

RAY E VANHUSS

DIRECTOR GOVERNMENT RELATIONS

GERBER PRODUCTS CO

445 STATE STREET

FREMONT. MI 49412

$616928-2267$

ERIC VAUGHN

PRE SI DEN T / CEO

RENEWABLE FUELS ASSOC

499 SO CAPITOL ST SW SUITE 420

WASHINGTON, DC 20003

$202484-9320$

PETER F VIEHWEG

SR DESIGN ENGINEER

ELGIN JOLIET \& EASTERN RY CO

1141 MAPLE RD

JOLIET. IL 60432

$815740-6573$

DON J VIOLETTE

PITNEY BOHES

380 MAIN AVE

NORHALK. CT 06852

$203853-7113$

GEORGE M VOGL

MANAGER OUALITY TECH $\&$ MFG SVC

CHURCH \& DWIGHT CO INC

$20 \mathrm{KINGSBR}$ IDGE RD PO BOX 369

PISCATAHAY. NJ 08854

$201885-1220 \times 167$

HAROLD HAINESS

OWNER/CUNSULTANT

HAROLD WAINESS \& ASSOCIATES

464 CENTRAL

NORTHFIELD. IL 60093

$312446-2402$

YOSHIYUK I WAKI SAKA

$R$ AND D MGR

KUBOTA AMERICA CORPORAIION

CHRYSLER BLOG 405 LEXINGTON AV

NEW YORK, NY 10174

$212490-8050$

DONALD R WALL

GREATER NY METRO FODDS

275 MADISON AVE

NEW YORK, NY 10016

$212689-8600$

J R WALLACE

REYNOLLDS METALS COMPANY

P 0 BOX 910

SHEFFIELD, AL 35660

$205386-6000$

JAMES K WALTERS

DIRECTOR MEASUREMENT

AMERICAN PETROLEUM INSTIIUTE

$1220 \mathrm{~L}$ ST NH

WASHINGTON. DC 20005

202 $682-8145$ 
RAY P WALTERS

MANAGER RETAIL MARKETING ENGR ASHLAND PETROLEUH COMPANY PO $80 \times 391$

ASHLAND: KY 41101

$606329-4421$

HAR YEY A WALZER

MANAGER CORP QUALITY ASSURANCE GENERAL FOODS CORPORATION 250 NOR TH ST HHITE PLAINS, NY 10625

$914335-5515$

HAROLD G WARP

4647 HEST AUGUSTA BLVD

CHICAGO IL 60651

$312261-5200$

KENNETH D WATKINS

OWNER

ACE SCALE COMPANY

1709 HWY $238 / P O$ BOX 387

ELHOOD, KS 66024

$913365-9398$

VINCENT ¿ WATTAI

OUALITY ASSURANCE SUPERVISOR

HER SHEY FOODS CORPORAT ION

19 E CHOCOLATE AVE

HER SHEY PA 17033

$717534-4686 \times 4686$

ELBERT $M$ WATTS

ADMINIS TRATOR OF CONTRACTS

AR I ZONA PUBLIC SVC $M$ S 1720

$411 N$ CENTRAL AVE/PO BOX 53999

PHOENIX, AZ 850723999

602 250-2806

SHERON E WEARY

OIRECTOR TECHNOLOGY AND DATA FLEXIBLE PACKAGING ASSOCIAT ION 1090 VERMONT AVE NW

HASHING TON, DC 20005

$202842-3880$

HARRY B WEAVER

DIRECTOR ENVIRONMENT ACTIVITY MOTOR VEHICLE MANUF ASSN

300 NEW CENTER BLDG

DETROIT, MI 48202

$313872-4311$

GEORGE WEBER

MER ICO INC

CARROLLTON, TX 75006

RICHARD H HEBER

METROLOGY LAB

MINNESOTA MINING \& MFG

BLDG 544-1-02

ST PAUL MN 55144

$612733-2674$

ROBERT L WEBER

MANAGER METROLOGY

LOCKHEED MISSILES \& SPACE CO $P$ O BOX 504 BLDG 195A 0/48-70 SUNNYVALE 408 CA 94086

RONALD HEESE

VAN DYKE SCALE INC

2825 RAYTOWN RO

KANSAS CITY, MO 64128

$816861=7191$
JAMES R WELLS

NEU YORK OIL HEATING ASSN

225 H 34 TH ST SUITE 1202

NEH YORK
212
$695-1380$

RAYMOND $R$ HELLS

VICE PRES - SALES

SENSITIVE MEASUREMENT INC

$P$ BOX 72

PEMBERTON: NJ 08068

$609894-2292$

EARL C WESTFALL

MANAGER TECHNICAL SVC SUPPORT

PITNEY BOWES

380 MAIN AVE

NORHALK, CT 06852

$203356-5541$

JAMES E HESTRICK

QC ENGINEER

GEO A HORMEL \& CO $80 \times 800$

AUSTIN. MN 55912

$507437-5754$

MICHAEL WHALEN

SR PROJECT TECHNOLOGIST

CAPE BRETON DEVELOPMENT CORP O BOX 2500/SYDNEY

NOVA SCOTIA

JOSEPH H WHEATLEY

SCALE TECHNITION

PEABODY COAL COMPANY

$P$ O BOX 545

GREENVILLE KY 42345

$502338-1960$.

RICHARD L WHIPPLE

REGULATORY ENG INEER

GILMARCO INC

3511 MARKET ST POB 22087

GREENSBORO, NC 27420

919 292-3011

JAMES $S$ WHITE

CONSULTANT ENVIRONMENT-HEALTH ARCO PETROLEUM PRODUCTS CO $B O X 2679$ TA

LOS ANGELES, CA 90051

$213486-8258$

JOHN R WHITE

TECHNIC I AN

FAIRBANKS WEIGHING DIVISION 718 RUBY

EXCELSIDR SPRINGS MO 64024

$816637-3401$

RAYMOND H WHITE

OWNER

WHITES PETROLEUM SERVICE

385 H ARNDT ST PO BOX 1241

FOND DU. W 54935

$414922-3930$

WILLIAM M WHITE

OWNER

NEW BEDFORD SCALE COMPANY

507 SMITH NECK RDAD

SOUTH DARTMOUTH. MA 02748

$617997-6730$

DAVID P WIDGEON

PRE SIDENT

WIDGEON ENTERPRISES INC

ROUTE 13 SOUTH P O BOX 8

LAUREL. DE 19956

$302875-5777$ 
MARRY L HILDASIN PHD

CONSULTANT GOVT RELATIONS

H P HOOD INC

500 RUTHERFORD AVE

BOSTON, MA O2129

GEORGE D WILKINSON

PRESIDENT

WILKINSON SCALE COMPANY INC

8800 WILKINSON BLVD

CHARLOTTE NC 28214

作

RANDALL F WILKINSON

DIRECTOR OF QUALITY ASSURANCE

TREE TOP INC

SELAH, WA 98942

$509697-7251 \times 621$

ROBERT R WILLIAMS

OWNER RMS PETROLEUM SVC \& EQUIP

HRLLIAAS $60 \times 328$

BEMIDJI. MN 56601

$218586-2038$

GEORGE D WILSON

VICE PRESIDENT

AMERICAN MEAT INSTITUTE

P D BOX 3556

WASHING TON, DC 20007

$202841-2400$

THOMAS A WILSON

MARKET ADMINISTRATOR

$N Y-N J$ MILK MARKETING AREA

708 THIRD AVENUE

NEH YORK, NY 10017

$212309-1600$

HARRISON B WILSON III

LEGAL DIVISION COUNSEL

ONE PROCTER \& GAMBLE PLZ/8599

CINCINNATI: OH 45202

$513983-5873$

GLENN $P$ WITTE

VICE PRESIDENT

INTL ASSN ICE CREAM MFG

888 16TH ST NH

WASHING TON DC 20006

ROD J WOLF

WYOMING OPERATIONS SUPERVISOR

WESTERN FUELS ASSOCIATION INC

203 CAREY AVE

GILLETTE, WY 82716

$307682-8051$

DA VID E WOLFERSBERGER

MANAGER OUALITY ASSURANCE

MONSANTO COMPANY (C2NJ)

$800 \mathrm{~N} L$ INDBERGH BLVD

ST LOUIS MO MO 63167

ANTHONY HOOD

QUALITY SYSTEMS SPECIALIST

M E M MARS

HIGH ST

HACKETTSTOWN. NJ 07840

$201850-2257$

JOHN E HOOD

SER VICE MANAGER

FAIRBANKS WEIGHING SYSTEMS DIV 408 MIAMI

KANSAS CITY, KS 66105
KEVIN D HOOD

CAL IBRATION SUPERVISOR AICRO MOTION INC

7070 WINCHESTER CIRCLE

BOULDER, CD 80301

$303530-8446$

DORSEY W WORLEY

PRESIDENT

BUSINESS EQUIPAENT CTR INC US ROUTE 4 BOX 192 CENTER RUTLAND V VT 05736

$802775-3349$

JOHN C WURGLER

MANAGER OUALITY ASSURANCE

KROGER CO DAIRY

$400 \mathrm{~S}$ SHOR TRIDGE RD

INDI ANAPOL IS. IN 46219

$317356-6800 \times 14$

GARY WYSE

DISTR IBUTION MANAGER

MICHIGAN PEAT CO

2243 MILFORD ST

HOUSTON. TX 77098

713 522-0711

KEVIN YAMAGUCH

MAR KETING MANAGER

CHUGAI INTERNATIONAL CORP

55 MALL DR

COMMACK, NY 11725

$516864-9700$

F G YARBROUGH

W $M$ CONSULTANT

ARROW INDUSTRIES INC

2277 HIGHWAY 36

ST PAUL. MN 55113

$214242-0525$

ERNIE H YASUKOCHI

O A MANAGER

LAWRYS FOOD INC

570 WEST AVENUE 26

LOS ANGELES. CA 90065

$213225-249 i$

GARY L YINGLING

PRESIDENT

FOOD \& DRUG LAW INSTITUTE

$1701 \mathrm{~K}$ ST NW SUITE 904

WASHINGTON, DC 20006

$202833-1601$

ALEXANDER YORGIADIS

VICE PRESIDENT

STRAINSERT COMPANY

1404 GARZA ST

ANAHEIM. CA 92804

$714827-3803$

JOHN C YOUNG

ASSISTANT/GENERAL COUNSEL

THOMAS J LIPTON INC

800 SYLVAN AVE

ENGLEWOOD CLIFFS. NJ 07632

JOHN W YOUNG

PRODUCT/DISTRIBUTION MANAGER

FRANKLIN ELECTRIC

PO BOX 666

LEVITTOHN. PA 19058

$215295-2000 \times 34$

RONALD C YOUNG

PRESIDENT

GENERAL METER SERVICE INC

123 4TH $80 \times 1$

RUSSELL. KS 67665

$913483-3758$ 
WAL TER J ZIELNICKI

SCALE AND WEIGHT COORDINATOR

1251 AVENUE OF THE AMERICAS

NEW YORK, NY 10020

$212489-9000 \times 542$

PAUL ZIEMER

AR I DIRECTOR / PACKAG ING

PRESIO PRODUCTS INCORPORATED

$B O X 2399$

APPLETON, HI 54913

$414739-9471$

GEORGE A 2 I MMER $R$

SUPERVI SOR ENVIRONMENTAL HLTH KENOSHA CITY HEALTH DEP

625 52ND ST

KENOSHA, WI 53140

$414656-8170$

A F ZIMAERMANN

C INC OUAL CONTR LAB DIV SOUTHAMPTON, PA 18966

ROBERT $F$ ZINDEL

$\sum_{\text {P }} \mathrm{R}_{\mathrm{O}} \mathrm{I}$ L EQUIPMENT CO

OREANA, IL 62554

WILLIAM H ZUSE

DIRECTOR MARKETING SERVICES

TR I ANGLE HACHINERY CO

6655 WEST DIVERSEY AVE

CHICAGO IL 60635

$312889-0200$ 
SECTION II

LISTING BY COMPANY 
RICHARD E BAILEY

1815 MARVIN GRIFFIN/POB 5641

AUG USTA. GA 30906

$404793-2190$

A M CANNAVA

14911 ROLLING RDG/PO BOX 2437

CHINO, GA 91708

\section{GARY CLARK}

MANAGER REGULATORY AFFAIRS

12200 DENTON DR

DALLAS, TX 75234

$214243-2321$

HENDRIK COLIJN

CONSULTING ENGINEER

423 FRANKL IN HTS DR

MONRDEV ILLE. PA 15146

$412372-8130$

HAROLO L F I SHER

2113 SOUTH SANTA FE

OKLAHOMA CITY. OK 73109

$405235-9212$

RONALD F I SHER

WEIGHTS \& MEASURES RETAIL INSP 8 ENR IGHT AVE

INDSOR. VT 05089

$802674-5355$

GEORGE C HARE

PRODUCT MANAGER

$P$ BOX 23099

MILWAUKEE, WI 53223

414 355-0400

BR I AN HOOVER

PRODUCT MARKETING ENGINEER

7070 WI NCHESTER CIRCLE

BOULDER - CO 80301

$303530-8534$

KNUD E JENSEN

961 MC CLELLAND ST

SALT LAKE UT 84105

$801364-9350$

MARK 0 JOHNSON

230 COLEMAN ST

RICE LAKE, WI 54868

$715234-9171$

ROBERT A MCDONALD

SERVICE MANAGER

P 0 BOX 1744

CUMBERLAND: MD 21502

$301759-3333$

RON MCVEY

PACKAG I NG/LABEL ING SPECIALIST

4900 YONGE ST SUITE 601

WILLOWDALE M2N6BB

JOHN R MITCHELL

11336 HEMBLEY ROAD

LOSS ALAMITOS. CA 90720

$213432-6981 \times 264$

HOWARD A NIELSEN

CONSULT ANT

2540 PAGE DRIVE

ALTADENA, CA 91001

$714739-1991$
RICHARD L NOLAN

OUALITY CONTROL MANAGER

62 HHITTEMORE AVE

CAMBR IDGE MA 02140

$617876-1400$

DAN 0 DONNELL

SERVICE MANAGER

$P$ D BOX AK

DES MOINES, IA 50302

CHARLES $K$ OVIAN

MGR WEIGHTS \& MEASURES DEPT

9 MONA VISTA AVE

RUTLAND: VT 05701

$802775-1571$

H STEFFEN PEISER

638 BLOSSOM DR

ROCKVILLE, MD 20850

301 762-6860

ROBERT ROSS

FLUID MEASUREMENT SPECIALIST

$P$ BOX 2040

TULSA. OK 74102

$918599-4200$

RICHARD SOUTHERS

6020 CHATSWORTH LANE

BETHESDA, MD 20814

$301530-8327$

HAROLD G WARP

4647 WEST AUGUSTA BLVD

312 CAGO: IL 60651

A E N ASSOCIATES

R C NICHOLAS

BOX I38A ROUTE

LOVINGSTON. VA 22949

A E P TEA COMPANY

RICHARD $R$ MC CALL

NATL DIR ENV HEALTH E SAFETY

2 PARAGON DR

MONTVALE NJ 07645

201 573-9700 X2577

A H EMERY COMPANY

CHESTER D BRADLEY

VICE PRESIDENT

RURAL RT 2 BOX 179

HEATHSVILLE VA 22473

$804580-4305 \times 37$

A H EMERY COMPANY

BRUCE A MURPHY

VICE PRESIDENT MARKETING

$P$ B BOX 608

NEW CANAAN: CT 06840

$203966-4551$

A J LADD WEIGHINGEPKGING SYSTM ANTHONY J LADD

CONSULTANT

255 N PORTAGE PATH SUITE 213

AKRON OH 44303

$216836-4569$

A I E S F RAILWAY

GRANT $V$ NIVEN

SYSTEM FUEL SUPERVISOR

$1001 \mathrm{~N}$ E A TCHISON

TOPEKA, KS 66616

$913235-0041 \times 7203$ 
AARROW EQUIPMENT COHPANY INC STEPHEN L CHARLTON PRESIDENT

$P 0$ BOX 665/HHY 52 ARMSTRONG PELHAM. AL 35124

$205663-5858$

ABBOTT LABORATOR IES

DOUGLAS M SMITH

MANAGER

1400 SHERIDAN RD/736-M4

NORTH CHICAGO. IL 60064

$312937-4929$

ABSCO SCALE CORP

HIKE R SCHEDWIN

VICE PRESIDENT

3408 INDIANOLA AVE

COLUMBUS 262643214

ACCURATE METERING SYSTEMS INC DONALD P ARNDTSEN

PRESIDENT

$1731-33$ CARMEN DR

ELK GROVE. IL 60007

ACCURATE SUPERIOR SCALE CO INC RAYMOND HEBENSTREIT

CONSULT TANT

3747 S JEFFERSON

ST LOUI S: MO 63118

ACE SCALE COMPANY
KENNETH D WATKINS

OWNER

1709 HWY 238/PO BOX 387

ELWOOD, KS 66024

$913365-9398$

ACME SCALE \& SERVICE COMPANY

GENE HAMMER

PRESIDENT

335 SOUTHHEST BL VD

KANSAS CITY MO 64108

$816842-2731$

ACME SCALE \& SUPPLY CO

JEFFREY D CANFIELD

PRESIDENT

5401 BUTLER ST

PITTSBURGH PA 15201

$412782-1808 \times 1$

ACME SCALE CO

GREN $M \frac{L}{G}$

1801 ADAMS AVE

SAN LEANORO, CA 94577

$415 \quad 638-5040$

AOOLPH COORS COMPANY

MARY J BOND

LIBRARIAN

MAIL BC 520

GOLDEN, CO 80401

$303277-3506$

AEM CORPORATION

DONALD J HINE

PE TROLEUM MEAS CONSULTANT

452 TEN TH ST

ELYRIA, OH 44035

216 $323-2041$

AGREX INC

LUKE RENZI

PROJECT LEADER

PIER A BERTH 211 AGREX BLK TER

LONG BEACH, CA 90802
AGR I EXPORI

GLENN R FEILNER

ELEVATOR MANAGER

1606 CLINTON DR

GALENA PARK: TX 77547

$713672-2536 \times 52$

AGR I-ELECTRONICS SYSTEMS ING ROBERT H BETTINGER

PRE SI DENT

12601 ECKEL ROAD

PERRYSBURG OH 43551

AGR I-MARK INC

M JEFFREY BLOOM

QUALITY CONTROL MANAGER

P O BOX 5800

LAWRENCE MA 01842

AGHAY INC

ALTON C TRUBEY

QUALITY CONTROL MGR FERT PROD

978 LOUCKS MILL RO

YORK. PA 17402

$717846-1286$

AGWAY INC MOTOR TRANSPORTATION JEFFREY A SLACK

PRODUCJION MANAGER

44 RI VER ST

CORTLAND. NY 13045

$607753-309$

AIRCO INDUSTRIAL GASES

KENNETH NUGENI

DISTRIBUTION ENGINEER

FOSTORIA, OH 44830

AIRHAY SCALE MFG CO INC RAYMONO G HARR IS

NATIONAL SALES MANAGER

10630 SO SANTA FE AVE

SOUTH GATE. CA 90280

213 563-5700

AL ELECTRONIC CASH REGISTER

E LEE HIGGINBOTHAM

25 WEST OXMOOR RD

BIRMINGHAM, AL 35209

ALLAN U BEVIER CO

GREG AYERS

OPERATIONS MANAGER

RD 3 BOX $85 B$

DELMAR MD 21875

$301546-3293$

ALLEN MILLER SCALES

BRUCE A MILLER

5926 MANN ST

LOUISVILLE. OH 44641

$216875-4179$

ALPO PETFOOOS INC

ROBERT HUTCHINSON

PO BOX 2187 ROUTE $309 / P O P E$ RD

ALLENTONN, PA 18001

AMAX COAL COMPANY

CLARENCE N CROCKER

MGR TECHNICAL SERVICES

105 S MER IOIAN ST P D BOX 967

INDIANAPOL IS. IN 46206

$317266-2824$ 
AMEACON INC

JAMES $\checkmark$ MOTSINGER

PRESIDENT

13703 THERMAL DR

AUSTIN TX 78728

$51225 i-3483$

AMER TEXTILE MFGRS INSTITUTE

HARDY B POOLE

ASST DIR-GYT REL - REGULATORY

1101 CONNECTICUT AVE HOHOO

HASHING TON, DC 20036

202862-0519

AMERICAN CALIBRATION/TEST INC THEODORE F MOONEY

PRESIDENT

178 WALNUT ST

LAWRENCE, MA 01841

$617686-8652$

AMERICAN CRYSTAL SUGAR CO

JERRY STANKORB

GENERAL CHEMIST

101 NOR TH THIRD ST

MOORHEAD, MN 56560

$218236-4400$

AMERICAN DRY MILK INSTITUTE

HARREN S CLARK JR

EXECUTI VE DIRECTOR

$130 \mathrm{~N}$ FRANKL IN ST

CHICAGO, IL 60606

$312782-4888$

AMERICAN FINE FOODS INC

RICHARD C COVINGTON

MANAGER QUALITY CONTROL

$P$ D BOX 460

PAYETTE, ID 83661

$208642-9061$

AMERICAN FROZEN FOOD INSTITUTE HUGH W SYMONS

VICE PRESIDENT RET SERVICES

1700 OLD MEADOH RD

MCLEAN YA 22102

$703821-0770$

AMERICAN GREETINGS CORPORATION ALLAN J GOODFELLOW

GENERAL COUNSEL AND SECRETARY

10500 AMER ICAN RD

CLEVELAND O OH 44144

AMERICAN MEAT INSTI TUTE

GEORGE D WILSON

VICE PRESIDENT

$P$ O $80 X 3556$

WASHINGTON, DC 20007

$202841-2400$

AMER ICAN METER CO

RICHARD H SCHIEBER

SENIOR TECHNICAL REPR

13500 P HILMONT AVE

PHILADELPHIA. PA 19116

$215673-2100$

AMERICAN NATIONAL METRIC COUNC ROBERT J PETERSEN

PROGRAM MANAGER

1010 VERMONT AVE N

MASHINGTON. DC 20005

$202628-5757$

AMERICAN PAPER INSTITUTE

\section{ROGER B BOGNAR}

MANAGER TISSUE DIVISION

260 MADISON AVE

NEW YORK. NY 10016
AMERICAN PETROLEUM INSTITUTE EARL A THOMPSON

MARKETING DEPARTHENT

$1220 \mathrm{~L} S \mathrm{NW}$

HASHINGTON, DC 20005
$202682-8230$

AMER ICAN PETROLEUM INSTITUTE JAMES $K$ WALTERS

DIRECTOR MEASUREMENT

$1220 \mathrm{~L} S T$ NH

WASHINGTON. DC 20005

$202682-8145$

AMERICAN YARN SPINNERS ASSOC JIM H CONNER

EXECUTIVE VICE PRESIDENT

PO BOX 99

GASTONIA. NC 28053

$704867-7201$

AMF UNION MACHINERY DIVISION P O SEILING

RQJECINEER

RICHMOND, VA

$804355-7961$

23227

AMOCO FOAM PRODUCTS CO

TIMOTHY R GAVIN

QUALITY ASSURANCE MANAGER

2907 LOG CABIN DR

SHYRNA, GA 30080

$40435 i-515 i$

AMOCO OIL CO

VIRGIL R HAMMOND

MAINIENANCE SUPERVISOR

$114 \mathrm{E}$ ARMOUR BLVD

KANSAS CITY, MO 64111

$913967-0133$

AMOCO OIL COMPANY

GERALD H ONEILL

DISTR ICT MAINTENANCE ENGINEER 150 S WARNER RD SUITE 432

KING OF, PA 19406

$215254-6100$

AMOCO DIL COMPANY

AMA SCHRAMM

$M C$ 1444A/PO $80 \times 5910-A$

CHICAGO, IL 60680

AMSTAR CORP

WAL TER J Z IELNICKI

SCALE AND WEIGHT COORDINATOR

1251 AVENUE OF THE AMER ICAS

NEW YORK. NY 10020

212 489-9000 X542

ANALYTICAL BALANCE SERVICE

RALPH J CARNEY

105 BRADLEY CIRCLE

DURHAM: NC 2

ANDERSON CLAYTON FOODS

T A BURCH

3333 N CENTRAL EXPWY

RICHARDSON, TX 75080

$214231-6121$

ANOERSON PEAT-ORGANIC COMPOST F TAMBL INGSON

2013 S ANTHONY BLVD

FT HAYNE, IN 46803 
ANDREW BOWMAN CO INC ANDREW G BOWMAN

OWNER

$85 T$ SO HOFFMAN LANE

CENTRAL ISLIP, NY 11722

$516234-6669$

ANGELL EOUIPMENT COMPANY INC ROBERT $J$ ANGELL

PRESIDENT

PO BOX 667

PELHAM. AL 35124

$205663-6706$

ANHEUSER-BUSCH INC

CARL E DARIGO

DIRECTOR CORPORATE QUAL ASSUR

ONE BUSCH PLACE

ST LOUIS. MO 63118

$314577-3966$

ANNISTON PUMP SHOP INC

CHARLES BROWN SR

VICE PRESIDENT

2800 HI WAY $431 \mathrm{~N}$ PO BOX 1198

ANNISTON, AL 36201

$205820-2980$

ARCO CHEMICAL COMPANY

KAREN S EBLE

1500 MARKET ST C $S-3301 \mathrm{G}$

PHILADELPHIA: PA 19101

$215557-3635$

ARCO CHEMICAL COMPANY

JORGE L FAZ

DIRECTOR GQVT \& INDUSTRY AFFRS

1500 MARKET ST

PHILADELPHIA. PA 19101

ARCO COAL SALES COMPANY

GLEN W NEWTON

MANAGER TRANSPORT ATION

555 SEVENTEENTH ST

DENVER, CO 80202

$303 \quad 293-7785$

ARCO PETROLEUM PRODUCTS CO

JAMES S WHITE

CONSUL TANT EN VIRONMENT - HEALTH

$B O X 2679$ IA

LOS ANGELES. CA 90051

ARI ZONA PUBLIC SVC M S 1720

ADMINISTRATOR OF CONTRACTS

$411 N$ CENTRAL AVE/PO BOX 53999

PHOENIX. AZ 850723999

$602250-2806$

ARKANSAS OIL MARKETERS ASSOC

ROBERT C ARNOLD

EXECUT I VE VICE PRESIDENT

$P$ BOX 229

LITTLE ROCK, AR 72203

ARKANSAS SCALES INC

DONALD W DYCUS

OWNER

ROUTE 8 BOX 156

JONESBORO: AR 72401

$501935-0505$

ARKSTROM INDUSTRIES INC

AARON D FRANZBLAU

PRESIDENT

415 AVON AVE

NEWARK. NJ 07108

$201243-1410$
ARROW INDUSTRIES INC

F G YARBROUGH

E M CONSULTANT

2277 HIGHHAY 36

ST PAUL. MN 55113

$214242-0525$

ASEA INC

BJORN THELEMARK

GEN MGR-PRESSDOCTORESENSOR DIV

ONE ODELL TERRACE

YONKERS, NY 10701

$914428-6000$

ASHLAND PETROLEUM COMPANY

RAY P WALTERS

MANAGER RETAIL MARKETING ENGR PO BOX 391

ASHLAND, KY 41101

606 329-4421

ASSN OF ANALYTICAL CHEMISTS EUGENE H HOLEMAN

STATE LIAISON REPRESENTATIVE

276 HARDING PLACE

NASHVILLE, TN 37205

$615352-3710$

ASSOCIATION OF AMERICAN RR JOHN J ROB INSON

SR ASSISTANT VICE PRESIDENT

50 F ST NH

WASHINGTON. DC 20001

202 293-4144

ASTM

EARL R SULLIVAN

DEPUTY DIRECTOR

1916 RACE STREET

PHILAOELPHIA, PA 19103

$215 \quad 299-5514$

AT $\&$ SF RWY CO

J 0 BAKER

ASST ENGINEER BALLAST SCALES

$4100 \mathrm{~S}$ KEOLIE AVENUE

CHICAGO. IL 60632

$312890-4114 \times 2115$

ATLANTIC RICHFIELD COMPANY

RICHARD J CUNNINGHAM

ENGR CONST \& MAINI

515 SDUTH FLOWER ROOM 1809

LOS ANGELES. CA 9007 I

$213486-2283$

AUTOMATIC MEASUREMENT TECHNOL JACK R CALDICOTI

PRESIDENT

IOOO RANO ROAO BLOG 111

HAUCONDA. IL 60084

$312526-0808$

AUTOWEIGH CO

JAMES A MACFARL ANE

VICE PRESI OENI

PO $80 \times 4017$

MODESTO. CA 95350

$209526-3557 \times 9$

8-P METER IESTING CO INC

ROGER H OLDS

PRESIDENT

608 NORTHSIOE DR

ENTERPR ISE AL 36330

$205347-8247$

BABSON BROS CO

R D BAR ILE IT

MGR REFRIG ENC

2100 S YORK RO

OAK BROOK. IL 60521 
BALLS SUPER FOODS INC

HAROLD SPILKER

MEAT MERCHANDISER

5300 SPEAKER ROAD

KANSAS CITY, KS 66106

$913321-4223 \times 50$

BANKHEAD ENTERPR I SES INC

WILLIAM E TRUMBO

CHIEF ENG INEER

PO BOX 93006 MARTECH STATION

ATLANTA GA 30318

$404894-7911$

BEATRICE GROCERY GROUP

CHIP KLDOS

LAB MANAGER - R \& D

1645 VALENCIA DR

FULLERTON, CA 926333899

$714680-1098 \times 1098$

BEATRICE GROCERY GROUP

AR T MI SENER

OC MANAGER

$3327 \mathrm{~W} 47 \mathrm{IH} P L$

CHICAGO? IL 60632

BEATRICE MEATS INC

RICHARD L JOHNSON

MANAGER OUALITY CONTROL

1919 SWIFT DR

OAK BROOK: IL 605229010
$312850-2825$

BEECH AIRCRAFT CORPORATION

DWANE L BAILEY

CHIEF OF WEIGHT CONTROL

9709 E CENTRAL AVE

WICHITA. KS 67201

$316681-7875$

BENJAMIN MOORE \& CO

DEBRA DATTILO

CHEMIST-REGULATORY-AFFAIRS

134 LISTER AVE

NEHARK, NJ 07105

$201344-1200 \times 42$

BENNETT PUMP CO

ROBERT L FONGER

SEN IOR TECHNICION

2740 HOOD ST

MUSKEGON, MI 49444

616 733-1302

BERKEL INC

TOM DE RYKE

MGR ENGINEERING AND PROD DEVEL

1 BERKEL DRIVE

LAPORTE. IN 46350

BERKEL INC

ROBERT MURRAY

REGULATORY AGENCY COORDINATOR

ONE BERKEL DRIVE

LAPORTE. IN 46350

$219326-7000$

\section{BERKEL INC}

MARK RODGER S

PROJECT ENG INEER

ONE BERKEL DR

LAPORTE IN 46350

$219326-7000$

BERNAT YARN \& CRAFT CORP

ROBERT M GODBOUT

OUALITY CONTROL

DEPOT \& MENDON ST

UXBR IDGE, MA 01569

$617278-2414$
BERNE SCALE COMPANY

CHR ISTOPHER P BERNE

PRESI DENT

2206 EDGEWODD AVE $S$

ST LOUIS, MN 55426

$612544-2422$

BERNZOMATIC DIV NEWELL COS

ROGER L MAXON

BERNZOMATIC CORPORATION

ONE BERNZOMATIC DR

MEDINA. NY 14103

BILL RUSSELL OIL CO INC BILL RUSSELL

PRESIDENT

215 EAST 9TH

RECTOR: AR 72461
$501595-2427$

BILLS PUMP \& REPAIR
BILL EDMAN
OWNER
RR2 BOX 259-H
IAWRENCE KS 66046
$913841-1758$

BIZERBA WERKE WILHELM KRAUT KG

PETER JUNG

SEN IOR ENG INEER

WILHELM KRAUTSTRASSE 41

D-7460 BAL I NGEN

BJ-TITAN SERVICES

MARK A OLSBERG

SENIOR PROJECT ENGINEER

11211 WEST FM 2920

TOMBALL. TX 77375

$713351-8131$

BLACK \& DECKER INC

THOMAS L RAWL INGS

SENIOR PACKAGE SPECIALIST

6 ARMSIRONG ROAD

SHELTON, CT 06484

$203926-3364$

BLACK PRODUCTS COMPANY

JOHN M VAN PERNIS

VICE-PRES MACHINE DIV

13513 S CALUMET AVE

CHICAGO, IL 60627

$312 \quad 468-9700$

BLH ELECTRONICS

KHAL IL D HAKER

75 SHAWMUT INDUSTR IAL PARK

CANTON. MA 02021

$61782 \mathrm{i}-2000$

BLUE FLAME GAS CORP

ASS ISTANT TO THE PRESIDENT

PO BOX 376

BLUFFION, IN 46714

$219824-3000$

BLUFF CITY SCALE COMPANY

BDBBY K LEWIS

OWNER

2856-14 LAMBS PLACE

MEMPHIS. TN 38118

$901365-1194$

BORDEN INC

JOHN $F$ THUERMER

ASSOC DIR OA \& COMPIIANCE-FOOD

960 KINGSMILL PARKWAY

COLUMBUS: OH 43229

$614225-4977$ 
BORDEN INC LAW DEPT BB-27 JEROME R SCHINDLER

ATTORNEY

P O BOX 2478

COLUMBUS OH 43216

$614225-4336$

BORG WARNER CHEMICALS

JAMES BOGGS

MANAGER OF ENERGY \& UTILITIES

PO BOX 658

OTTAWA. IL 61350

BRADY ENTERPRISES INC

MICHAEL SANNA

MANAGER OUALITY MANAGEMENT

167 MOQRE ROAD

EAST WEYMOUTH, MA 02189

$617337-5000 \times 21$

BRASWELL SCALE \& EQUIPMENT CO

JIM C DWNBEY

VICE PRESIDENT

$P O B O X 5422$

ASHEVILLE: NC 28813

$704274-3771$

BRINKMANN INSTRUMENTS CO

BERND G LUDEWIG

GEN MGR

CANTIAGUE RD

WESTBURY: NY 11590

516 -S $\times 216$

BRISTOL-MYERS PRODUCTS

WILLIAM C FREY

225 LONG AVE

HILLSIDE NJ 07207

$201926-9304$

BR I STOL-MYERS PRODUCTS

JOSEPH L LAHRENCE

PACKAGE ENGINEER ING MANAGER

225 LONG AVENUE

HILLSIDE NJ 07207

$201926-9304$

BROCKWAY INC

RAY FI SCHER

PRODUCT COORDINATOR

BRDCKWAY, PA 15824

$814268-3015$

BROOKS INSTRUMENT DIVISION

BARRIE $L$ BLOSER

CHIEF ENG INEER

HWY 301 NORTH BOX 450

STATESBORO GA 30458

$912764-5471 \times 284$

BUCKEYE GAS PRODUCTS CO

GLENN E MACE 7130 SOUTH LEWIS PO BOX 3478 TULSA: OK 74101

$918496-7535$

BUFFALO SCALE \& SUPPLY COMPANY JOHN R RIZZO VICE PRESIDENT

2609 CHARTIERS AVE

PITTSBURGH, PA 15204

$412331-3335$

BUREAU INT METROLOGIE LEGALE

BERNARD ATHANE

DIRECTOR

11 RUE TURGOT

PARIS 75009
BURLINGTON NORTHERN RAILROAD NORMAN R BRUCKER

REGIDNAL SCALE INSPECTOR 176 EAST 5 TH ST BOX 64960

ST PAUL: MN 55164
$612298-2605$

BURL INGTON NOR THERN RAILROAD JAMES W OAVIOSON

ASSISTANT CHIEF ENGINEER

$P$ O BOX 29136

OVERLAND PARK. KS 66201

$913661-4238$

BURLINGTON NORTHERN RAILROAD RONALD J HOSTETLER

DENVER REGION SCALE INSPECTOR $R R \quad B O X 100$

MILFORD. NE 68405

$402761-2623$

BURLINGTON NORTHERN RAILROAD DAVID PHILLIPS

INSPECTOR

1300 EAST H ST APT 29

MCC OOK. NE 69001

$308345-7547 \times 283$

BURMAH-CASTROL INC

GEORGE E GOODR I CH

ENVIRONMENIAL \& SAFETY MANAGER 401 HACKENSACK AVE

HACKENSACK. NJ 07601

$201488-1080 \times 62$

BUSINESS EOUIPMENT CTR INC DORSEY W WORLEY

PRESIDENT

US RDUTE \& BOX 192

CENTER RUTLAND. VT 05736

$802775-3349$

CEE SCALES \& EQUIPMENT CO

CHARL IE ELLIS

OWNER

PO BOX 700 (104 PI GIBSON RD) RAYMOND, MS 39154

$601857-5143$

CALIBRATION DIVISION OA-CB

JAMES R HINCHEY

CHIEF BASIC STDS BRANCH

U S ARMY WHITE SANDS MISSILE

RANGE NM 88002

$505678-1720$

CAL IFORN IA ALMOND GROHERS EXCH DONALD A BROWN

PROJECT ENGINEER

PO BOX 1768

SACRAMENTO, CA 95808

$916446-8386$

CALIFORNIA BREWERS ASSOCIAIION GEORGE USOSKE

EXECUTIVE VICE PRESIDENI

235 MONTGOMERY STREFT 508

SAN FRANCISCO. CA 94104

$415421-7747$

CAL IFDRNIA LEAGUE FONO PROCESS LAWRENCE K TABER

PRESIDEN I

1007 L ST

SACRAMENTO. CA 95814

$916444-9260$

CALLIS-THDMPSDN INC

WILLIAM G THOMPSON

UP

PO BOX 177

HARRINGION, DE 19952 
CALRECQ

PHIL CRAIN

DIRECTOR QUALITY AS SUR ANCE

8015 VAN NUYS BLVD

VAN NUYS, CA 91412

$818787-7820 \times 225$

\section{CAMCAL COMPANY}

JOHN L CARLTON

PRESIDENT

4000 AIRPORT HAY SOUTH

SEATTLE, WA 98108

$206682-5416$

CAMPBELL SOUP CO

R KEVIN CHUMNEY

MGR GOVT REGULATIONS

CAMPBELL PLACE

CAMDEN, NJ 08101

CANADIAN GRAIN COMMISSION

C J DEMPSTER

GRAIN RESEARCH LABORATORY

$1404-303$ MAIN SI

HINNIPEG R3C

CANADIAN GRAIN COMMISSION

ROBERT A MCLEOD

ACT ING DEP DIR AUDI TS-SCALES

BO0-303 MAIN ST

WINNIPEG MAN

CAPE BRETON DEVELOPMENT CORP

MICHAEL HHALEN

SR PROJECT TECHNOLOGIST

$P$ O BOX 2500/SYDNEY

NOVA SCOTIA

CAPIIOL SCALE CO INC

MAR ILYN SMITH

TREASURER

927 SH WASHINGTON ST

PEORIA, IL 61602

$309676-0865$

CARDINAL SCALE CO

HILLIAM V GOODPASTER

VICE PRESIDENT

$1610 \mathrm{~N}$ C ST

SACRAMENTO, CA 95814

$916441-0178$

CARDINAL SCALE MFG CO

EA E BUR MAN

$P$ O BOX 24-061

APPLE VALLEY, MN 55124

$612432-2100$

CARDINAL SCALE MFG CO

W TERRY JAMES

VICE PRES ENG INEERING SERVICES

203 E DAUGHERTY

WEBB CI TY MO 64870

$417673-4631 \times 20$

CAROINAL SCALE MFG CO

H P PER R

PRESIDENT

$P$ OBOX 151

HEBB CITY. MO 64870

$417673-4631$

CARGILL INC

JOHN A JOHNSTON

P O BOX 930O/CMO

MINNEAPOLIS. MN 55440

$612721-8531$
CARPET AND RUG INSTITUTE

JUDY CLINE

ASST TO TECHNICAL DIRECTOR

PO BOX 2048

DAL TON: GA 30720

CASE SHAYNE CO INC

$\checkmark$ ICKI NASH

OUALITY CONTROL MANAGER

$1632 \mathrm{E} 4 \mathrm{TH}$ ST

SANTA ANA. CA 92702

$714558-8 B 61$

CASTLE \& COOKE INC

ANDREW B MOORE

QUAL I IY AS SURANCE AUDITOR

$P$ O BOX $5130 / 100$ PARK PLAZA CT

SAN JOSE, CA 95150

$408279-8750 \times 4305$

CATO OIL AND GREASE COMPANY

RALPH JENKS

PRESIDENT

$915 \mathrm{~N}$ EASTERN

OKL AHOMA CITY, OK 73117

$405424-3311 \times 221$

CENEX

RALPH S SCHLETTY

PETROLEUM EOUI PMENT

BOX 64089

SI PAUL:MN 55164

CENTRAL SCALE INC

TED DONALDSON

PRE SIDEN T

4915 E 16TH ST

IND IANAPOLIS, IN 46201

$317356-8005$

CHADWELL AND KAYSER

MERRILL S THOMPSON

$\checkmark$ ICE PRESIDENT \& PARTNER

P O BOX 8500

BRIDGETON, IN 47836

$317548-2202$

CHARLOTTE SCALE CO INC

MAR IIN E OEHLER

PRESIDENT

1510 AMERON DR IVE

CHARLOTTE. NC 28206

704 376-8496

CHESEBROUGH-PONDS INC

DAVID E HAM

PROJECT PLANNING MANAGER

33 BENEDICT PLACE

GREENWICH. CT 06830

$203661-2000 \times 1358$

CHEVRON USA INC

M HALBLE IB

SEN IOR ENG INEER

P O BOX 7006 ROOM 267 ?

SAN FRANCISCO. CA 94120

CHICAGO AND NORTHWESTERN TRANS

WILLIAM G GEME INER

SCALE ENGINEER

ONE NOR THWESTERN CENTER

CHICAGO, IL 60606

$317559-6133 \times 6610$

CHOCOLATE MANUFACTURERS ASSN

RICHARD T O CONNELL

PRESIDEN

7900 WES IPARK DR

MCLEAN. VA 22102

703 790-5011 
CHUGAI INTERNATIONAL CORP

KE VIN YAMAGUCHI

MARKETI NG MANAGER

55 MALL DR

COMMACK, NY 11725

$516 \quad 864-9700$

CHURCH \& DWIGHT CO INC

GEORGE $M$ VOGL

MANAGER OUAL ITY TECH \& MFG SVC

20 KING SBRIDGE RD PO BOX 369

PI SCATAWAY. NJ 08854

$201885-1220 \times 167$

CHURCH AND DWIGHT CO INC

MICHAEL R DOTY

OUALITY CONTROL SUPERVISOR

BOX 123

GREEN RIVER, WY 82935

$307875-2233 \times 124$

CI BA GE IGY CORPORATION

LEE HEINR I CHS

STAFF CHEMIST

PR EENSBORO: NC 27409
919 292-7100

CIVIL ENGINEERING ECJ 4.2

CLYDE E LEE

PROFESSOR

UNIV OF TEXAS AI AUSTIN

AUSTIN, TX 78712

$512471-4549$

CLOROX COMPANY

TERRY C GARDNER

TECH SVCS COORDINATOR

PO BOX 493

PLEASANTON. CA 94566

$415462-2100 \times 396$

CLOROX COMPANY

BARNEY T HOPKINS

MFG SER VICES SUPERVISOR

$P$ O BOX 493

PLEASANTON, CA 94566

$415847-6130$

C.H I COR PORATION

KEN COSBY

MANAGER COMPUTER CONTROLS DIV $P$ D BOX 1985

OKLAHOMA CITY. OK 73101

$405787-6020$

\section{CORPORATION}

RAY S IOZIOR

GEN MGR WEIGHING EQUIPMENT DIV P O BOX 1985

OKLAHOMA CITY, OK 73101

$405787-6020$

CMI HEIGHING EQUIPMENT

LYNN A MARVEL

ENG INEER ING MANAGER

6101 RENO SUITE 800

DKLAHOMA CITY, OK 73127

$405495-6830 \times 308$

\section{IDEARBORN}

JEFFREY B DAYIES

MARKETING DIRECTOR 82 O 8 IAFAYETTE RD BLDG 1 \#203

$603772-9791$

COASTAL STATES ENERGY CO

ARLIS SCHULTE

TRAFF IC MANAGER

NINE GR EENHAY PLAZA

HOUSTON. TX 77046
COATS ANO CLARK INC CONRAD JOHNSON MANAGER OUALITY ASSURANCE $P$ O BOX 670

TOCCOA. GA 30577

$404886-2141$

COCA COLA COMPANY MICHAEL J GILROY FOOD \& DRUG SENIOR COUNSEL $P$ D DRAWER 1734 ATLANTA, GA 30301 404 676-2121

COCA-COLA COMPANY

IRVING BEL L

SR EXECUTIVE STAFF REPR

PO DRAWER 1734

ATL ANTA, GA 30301

$404676-2623$

COCA-COLA COMPANY

ROBERT A LESTER

ATTDRNEY

PO DRAWER 1734

ATLANTA, GA 30301

$404676-2530$

COLGATE PALMOL IVE

BILL FOWLE

JEFFERSONVILLE. IN 47130

$812283-6611$

COLGATE PALMOL IVE CO

KENNETH C APPELL

DIREC TOR-OUAL ITY ASSURANCE

300 PARK AVE

NEW YORK. NY 10022

$212310-2022$

COLORADO-UTE ELECTRIC ASSN INC KENT E DAVENPORT

ENG INEER FUELS

P $\mathrm{BOX} 1149$

MONTROSE, CO 81402

$303249-4501$

COLUMBIA GRAIN INC

ROGER N ANDERSON

VICE PRESIDENT

$P$ O BOX 03370

PORTLAND. OR 97203

$503286-9681$

COLUMBIAN CHEMICALS COMPANY

JOHN R RIODELL

MANAGFR TRAFFIC

1600 PARKWOOD CIRCLE NH RM 400 A TLANTA. GA 30339

$404951-5700$

COMMERCIAL SCALE CO INC

DAVID C ROBBINS

PRESIDEN T

P O BOX 204

INDIAN ORCHARD, MA 01151

$413543-2290$

COMPETROL INC

ROY HAIGH

SALES MANAGER

8714 LION ST SUITE D

CUCAMONGA. CA 91730

$714987-0808$

COMPUWF I GH CORP

HAROLD H ECKE

PRE SI DENT

PO BOX 4517 YALESVILLE STATION WALL INGF ORD, CJ 06492

$203284-9184$ 
CONAGRA INC

ROBERT J NEWMAN

VP-MANUFACTUR ING

ONE CENTRAL PARK PLAZA

OMAHA, NE 68102

$402978-4050$

CONDON OIL COMPANY ROBERT F REISER JR OPERATI ONS MANAGER

126 E JACKSON ST

RI PON, WI 54971

$414748-3186 \times 41$

CONRAD FAFARD INC

HER VE H FAFARD

PRE SI DENT

PO BOX 3033

SPR INGF IELD, MA 01101

$413786-4343$

CONRAIL

JOE F KESSLER

SCALE INSPECTOR

501 E MICHIGAN

JACKSON. MI 49201

$517789-6261$

CONRAIL

C THOMAS PICTON

SYSTEM SUPERYISOR SCALE INSPEC 6 PENN CENTER ROOM 1634 PHILADE LPHIA. PA 19104 $215977-1617$

CONSOL IDATION COAL CO CHARLES J DUNAWAY

STAFF ENGINEER-TECH MARKETING 1800 WASHINGTON ROAD PITTSBURG PA 15241

$412831-4388$

CONSUMER \& CORPORATE AFFAIRS ROBERT BRUCE

ACT ING CHIEF WTS AND MEASURES

OT TAWA ONT

$613996-3035$

CONSUMER E CORPORATE AFFAIRS GERRY A JOROWSKI

PROGRAM OFFICER

50 VICTORIA PLACE DU PORTAGE

HULL QUEBEC

$819997-1177$

CONSUMER AFFAIRS BUREAU

RONALD $J$ CRAHFORD

CHIEF INSPECTOR $W \& M$

$P$ O BOX 252

BR I SBANE N

CONSUMER TRADING STANDARDS DPT CLIVE A HOWARD-LUCK

DIRECTOR

STRATHCLYDE HOUSE INDIA ST

GLASGOW G24PF

CONSUMERS POWER CO

GERALD $R$ BURGER

COAL SUPPLY COORDINATOR

1945 W PARNALL RD

JACKSON. MI 49201

$517788-0114$

CONTINENTAL GRAIN CO

DONALD J BAIN

VICE PRESIDENT-COMPLIANCE

277 PARK AVE

NEH YORK. NY 10017

$212 \quad 826-5532$
CONTINENTAL GRAIN CO

MACK BROWN

OPERATIONS MANAGER

5100 OAKLAND AVE

ST LOUIS. MO 63110

$314531-8300$

CONTINENTAL PIPE LINE COMPANY RICHARO N TENNILLE

MANAGER TECHNICAL DEVELOPMENT $P O B O X 2197$

HOUSTON. IX 77252

$713293-1776$

CONTINENTAL PIPELINE CO

JACK A L IERLY

SUPERVISOR CORROSION \& MEAS

7666 E 6IST SUITE 325

TUL SA. OK 74133

$918743-8803$

CONTINENTAL SCALE CORPORATION

WAL TER P KUSHMUK

MGR ENGI NEERING \& RESEARCH

7400 WEST $100 T H$ PLACE

BRIDGEVIEW: IL 60455

$312598-9100 \times 34$

CORRELLS SCALE SERVICE

RANDY PUGH

$\checkmark$ ICE PRESIDENT

RT 5 1450 NELSON RD

MOSES LAKE WA 98837

$509765-7754$

COUNSELOR COMPANY

$S$ ROBERT GUINTER

CHIEF PRODUCT DESIGN ENGINEER

2107 KI SHWAUKEE ST

ROCKF ORD: $1 \mathrm{~L} 61101$

COX FIXTURE \& SUPPLY INC

ROBERT N COX

PRES IDENT

3412 JACKSONVILLE HWY

NORTH LITTLE. AR T2117

$501945-6011$

CPC BEST FOODS

FRED J FANDERS

OAMGR

1120 COMMERCE AVE

UNION. NJ 07083

$201688-9000 \times 283$

CRANDALL FILLING MACHINERY INC KEITH A CRANDALL PO $80 \times 706$

BUFFALO. NY 14217

$716885-2228$

CROWN ZELLERBACH CORP

ROGER D KITCHING

PACKAGING MGR - CONSUMER PROD

ONE BUSH ST

SAN FRANCISCO, CA 94104

$415951-5568$

OAIRY/FOOD INDUSTRIES SUPPLY

THOMAS M GILMORE

TECHNICAL DIRECTOR

0245 EXECUTIVE BLVD

ROCKVILLE. MD 20852

$301984-1444$

DANIEL INDUSTRIES INC

JACK HAR SHMAN

MANAGER PRODUCT DEVELOPMENT

PO BOX 19097

HOUSTON: TX 77024 
DANTEST

E REPSTORFF HOLTVEG

DIREC TEUR

AMAGER BOULEVARD 115

DK 2300

DAR IGOLD INC

WILLIAM H BREWER

MGR RESEARCH \& DEVELOPMENT

P O BOX C19099

SEATTLE, WA 98119

$206284-7220$

DAT ACHE CKER / DTS

EDWARD H SONN

VP PRODUCT MARKETING

124 ACTDN ST

MAYNARD, MA 01754
$617897-3221 \times 2400$

DAVIS REFR IGERATION SVC INC

BILLY E DAVIS

PRESIDENI

1207 SO CHURCH ST

ROCKY MOUNT, NC 27801

DCA FOOD INDUSTRIES INC

JOSEPH M LAINO

CORP DIR OA \& REGULATORY CDMPL

919 THIRD AVE

NEW YDRK. NY 10022

$212371-6600 \times 300$

DEAN FOODS COMPANY

GEORGE A MUCK

VICE PRESIDENT RESEARCH \& DEV

1126 KI LBURN AVE

ROCKFORD, IL 61101

$815962-0647 \times 294$

DECATUR DANVILLE SCALE CO INC

CECIL J DAMERY

PRESIDENT

120 S WALL ST PO BDX 299

MACON, IL 62544

$217764-3341$

DEL GIODICE ASSOCIATES

VINCENT J DEL GIUDICE

CONSULT ANT

$6620 \mathrm{~N}$ WAUKESHA AVENUE

CHICAGO, IL 60646

$312763-1183 \times 3075$

DEL MDNTE CORPORATION

WILLIAM J SPAIN

DIRECTOR REGUL AFFRS ENERGY MG PO BOX 3575

SAN FRANCISCO, CA 94119

$415442-4610$

DELAVAL AGRICULTURE DIVISION

MAT TORMEY

MARKETING MGR COOLING/ENERGY

11100 NORTH CONGRESS AVE

KANSAS CITY, MO 64153

$816891-7700 \times 484$

DELTA SCALE INC

JAMES G AYERS

4525 MULLENS FORD ROAD

CHARLOTTE NC 28226

$704527-8256$

DEL TA SCALE INC

ADOLPH H GALINDO JR

10108 WDODVIEW CIRCLE

MATTHEWS: NC
DENVER $\&$ RID GRANDE WESTERN RR

KEITH W BRADLEY

ASST CHIEF ENGR-PLANNING

1515 ARAPAHDE ST PD BDX 5482

DENVER , CO 80217

$303629-5533 \times 2433$

DEPARTMENT OF LABOUR

THE LIBRAR IAN

PRIVATE BAG

WELL INGTON

DEPT OF PUBLIC 6 CDNS AFFAIRS JAMES A SERVIN

DIR CONSUMER AFFAIRS DIVISION

25 GRENFELL ST GRENFELL C.ENTRE ADELAIDE SA 2283211

$\times 247$

DERLEIN SCALES \& MFG INC JOHN CHESLEY

PRESIDENT

2425-0 S STOUGHTDN RD

MADISON. WI 53716

$608222-0606$

DESKIN SCALE CO INC

LEE C DESK IN

PRESIDEN

437 W MC GEE ST

SPRINGFIELD, MO 65804

$417883-0055$

DETERMAN WELDING \& TANK TDM DETERMAN

1241 72ND AV NE

MINNEAPDLIS. MN 55432

$612571-8110$

DETRDIT EDISON A-318 WSC

GER ARD B MACK

PRODUCTIUN SERVICES

2000 SECOND AVE

DETROIT. MI 48226

$313897-0115$

DETRDIT EDISON COMPANY

DARRY I BDYD

ELECTRONIC ENG INEERING IECH

6100 W WARREN

DEIROIT: MI 48210

$313897-119$

DFA OF CALIFORNIA

FRANK A MOSEBAR

PRESIDENT

$P$ O BDX $270 A$

SANTA CLARA. CA $9505 ?$

$408727-9302$

DICKEY-JOHN CORPORATION

DAVID B FUNK

PROJECT DEVELOPMENT FNGINFER

$155 \mathrm{~S}$ COUNTRY CLUB RD

AUBURN. IL 62615

$217438-337$

DJ INSIRUMENTS INCDRPORATED DAVID J FIRSI

PRESIDFNT

18 REPUBLIC RD

NORTH BILLERICA. MA 01862

$617667-5301$

DOCKENDDRF EQUIPMENT CD INC

DUANE MIKKELSEN

$830 \mathrm{E} I 5 \mathrm{TH}$ SI/PD $80 \times 1222$

SINUX FALLS. SD 57101 
DOVER CORP OPW DIVISION

G E MOORE

$\checkmark P$ ENG INEER ING

$P$ O BOX 405003

CINCINNATI, OH 45240

$513870-3208$

DOW CHEMICAL USA

ARTHUR SOLIS

WEIGHT SYSTEMS SPECIALIST

PO BOX 1398 LOVERIDGE ROAD

PITTSBURG, CA 94565

$415 \quad 432-5006$

DOW CORNING CORP

RALPH PRETZER

SUPERVISOR CORPORATE PACKAGING

MAIL \#112 P O BOX 1592

MI DLAND? MI 48640

$517496-4000$

DRACKET T COMPANY

DAVID J SAVAGE

DIRECTOR OF QUALITY ASSURANCE

5020 SPRING GROVE AVE

CINCINNATI, OH 45232

$513632-1406$

DUNBAR MANUFACTURING INC

HARVEY M LODGE

PRES IDENT

307 BROADWAY

SHANTON, OH 43558

$419244-3021$

\section{DUNCAN INDUSTRIES}

ALBERT A PESENT I

VICE PRESIDENT SALES

1701 GOLF ROAD SUITE 1100

ROLLING MEADOWS, IL 60008

$312952-2990 \times 210$

DUTCH METROLOGY SERVICE

J NIEUWLAND

CHIEF LEGAL/ JURIDICAL AFFAIRS

P O BOX 654

2600 AR

015569271

E E J GALLO WINERY

PAUL C THORPE

PO BOX 1130

MODESTO. CA 95353

$209521-3490 \times 3490$

E I DUPONT DE NEMOURS \& CO

$R$ N BUMGARDNER

901 DUPONT AVE

BELLE. WV 25015

$304949-4313$

E R SQUIBB \& SONS INC

AUGUSTUS E SYERS

GEORGES RD

NEW BRUNSWICK, NJ 08903

201 545-1300 $\times 3407$

E KNAUSS $E$ SON INC

E WILLIAM KNAUSS

VICE PRESIDENT

OUAKERTOWN, PA 18951

$215536-4220$

\section{EAGLE MICROSYSTEMS}

MICHAEL L SMITH

SALES MANAGER

RT $100-R D$ W

CHESTER SPRINGS PA 19425

$215458-5778$
EASTERN MILK PRODUCERS

KENNETH SLENTZ SR

QUALITY CONTROL FLD OPR MGR

$6567 \mathrm{KINNE}$ RD

SYRACUSE, NY 13214

$315446-0730 \times 36$

EASTERN SCALE COMPANY INC

LEONARD MATLOSZ

VICE PRESIDENT

1053 PENNSYLVANIA AVE

LINDEN, NJ 07036

$201486-4433$

EASTERN WEIGHING SYSTEMS INC JAMES A SCHRACK

PRESIDENT

BATH, PA 18014

$215759-2704$

EAU CLAIRE EQUIPMENT CO INC

PETER G HEACOX SR

OFF ICE MANAGER

2620 DAVEY ST

EAU CLAIRE WI 54703

$715 \quad 832-2987$

EOWARDS BAKING COMPANY

CHARLES H BROKAH

DIRECTOR OF PRODUCJ INTEGNITY

1 LEMON LANE

ATL ANTA. GA 30307

$404377-0511$

EDWAROS EQUIPMENT CO INC

VICKI EDWARDS

261-C W FLEMING DR

MORGANTON. NC 28655

$704 \quad 433-0650$

EGEG IDAHO INC

ROGER W ELLIS

MANAGER STANDARDS \& CAL LAB

PO BOX 1625

I DAHO FALLS. ID 83415

$208 \quad 526-2656$

ELECTROMETRICS CO

JAMES G BECKER

PRE SI DENT

6433 HAVELOCK AVE

IINCOLN, NE 68507

$402467-3541$

ELFC.TRONIC SCALES INTERNATL

HENRY C HARBERS

PRE S I DEN T

$P$ O BOX 3259

SAN LUIS CCA 93403

ELECTROSCALE CORP

DAVID CERVANTES

PROJECT ENGINEER

$P 0$ B 1786

SANTA KOSA: CA 95402

$707584-9720$

ELGIN JOLIET \& EASTFRN RY CO PETER F VIEHWEG

SR DESIGN ENGINEER

1141 MAPLE RD

$J$ OL IET. IL 60432

$815740-6573$

ELI LILLY ANO CO

LEROY A SPR INGMAN

MANAGER QUALITY CONTROL

307 EAST MCCARTY STREET

I NO IANAPOL IS. IN 46285

$317261-4686$ 
EL I ZABETH ARDEN INC

BARBARA LEVI ESQ
PARK AVE PLAZA 55 E $52 N D$ ST

NEW YORK. NY 10022

$212407-1112$

\section{EMARK CORPORATION}

BILL MÁHLER

ELECTRONIC SYSTEMS ENG INEER

11065 SORRENTO VALLEY CT

SAN DIEGO, CA 92121

$619457-1180 \times 312$

EAPRO PRODUCTS COMPANY INC PETER DE KORTE

PRESIDENT

357 MCLEAN BLVD

PATERSON, NJ 07513

$201279-1010$

ENG INEERED SYSTEMS INC

BRYAN L SHINNEY

MARKETING / SYSTEMS

TEMPE, AZ 85282

$602438-1362$

ENID BOARD OF TRADE

HAROLD L SCHULTZ

SUPERVI SOR OF WEI GHTS

PO BOX 17472309 NORTH $10 \mathrm{TH}$

ENID, OK 73701

$405233-1528$

\section{ENIERPRISE COMPANIES \\ MARY GE TTY}

OUALITY ASSURANCE DIRECTOR

1191 SOUTH HHEEL ING ROAD

WHEELING. IL 60090
$312541-9000$

EQUIPAENT CONTROLS CO

JAMES E BELL

PRESIDENT

4555 BERKELEY LAKE RD S

NORCROSS GA 30071

ERIE STRAYER COMPANY

HAMILTON W STRAYER

PRESIDENT

$P$ POX 1031

ERIE PA 16512

$814456-7001$

ERK SCALE CO INC

ALBERT ERK

OWNER

RT 4 BOX 262-B

FRANKLINTON, LA 70438

$504839-5660$

ERNIE GRAVES COMPANY INC

FRANK D GRAVES

PRESIDENT

201 S HOUSTON

TULSA. OK 74127

$918584-4707$

ESSELTE METO INC

D HOWARD RANDALL JR

TECHNICAL PRODUCT MANAGER

RANDOLPH. MA 02368

$617986-6200 \times 37$

ESSELTE MOREAU

GERARD SABIRON

DIR RESEARCH \& DEVELOPHENT

94100 SAINT
EX-CELLO MAIERIALS HANDLING CO STEVE J REYNOLDS

ELECTRICAL ENG INEER

PO BOX 6549 JOHNSON PKWY-AMES ST PAUL. HN 55106

$612776-8501$

EXACT WEIGHT SCALE

ROY W LUTLEY

VICF-PRESIDENT GENERAL MANAGER

1191 CRESTLAWN DR

MISSISSAUGA ONTARIO

$416 \quad 625-6220$

EXXON CHEMICAL AMERICAS

JOSEPH R MURPHY JR

MARKETING MANAGER

P 03272

HOUSTON. TX 77001

$713870-6178$

EXXON CO USA

JOHN R MCPHERSON

SENIOR STAFF ENGINEER

BOX 4388

HOUSTON. IX 77210

$713874-5203$

EXXON COMPANY USA

$G$ L GARTEI SER

MGR DISTRIBUTION \& ENGINEER ING $P 0$ BDX 4388

HOUSTON. IX 77210

$713656-6113$

EXXON COMPANY USA 38719

WILLIAM C STEWART

ENGINEER ING SERVICES COORD

$\mathrm{P} O \mathrm{BOX} 4388$

HOUSTON. TX 77210

$713656-6170$

FAIRBANKS WEIGHING DIV COLT IN DICK HURLEY

MGR REGUL COMPL IANCE

711 EAST ST JOHNSBURY RD

ST JOHNSBURY. VT 05819

$802748-5111 \times 349$

FAIRBANKS WEIGHING DIVISION

TAYLOR SOPER

PRESIDENT

COLT INDUSTRIES

ST JOHNSBURY. VT 05819

$802748-5111 \times 300$

FAIRBANKS WEIGHING DIVISION JOHN R WHITE

IECHN IC I AN

7 I8 RUBY

EXCEL SIOR SPRINGS. MO 64024

$816 \quad 637-3401$

FAIRBANKS WEIGHING SYSTEMS DIV JOHN E WOOD

SERVICE MANAGER

408 MIAMI

KANSAS CIIY. KS 66105

$913342-1077$

FANTASY FLAVORS INC

JACK C GROSSKOPF

TECHNICAL DIRECTOR

$24 W 051$ NORTH AVENUE

WHEATON. IL 60187

$312668-1000$

FAULTLESS RUBBER CO

ROGER JENNEY

CAL I BRAI ION COORDI NATOR

268 W 4 TH STREET

ASHLAND. OH 44805 
FEDERAL HIGHWAY ADM DOT

ED RUGENSTEIN

HWY ENGR HCT-1

HASHINGTON, DC 20590

$202426-6282$

FEL-PRO INCORPORATED ROBERT J MORRIS

YP ADVERTISING \& PUBLICATIONS $7450 \mathrm{~N}$ MCCORMICK BL VD SKOKIE, IL 60076

$312761-4500$

FERRANTI INDIANA INC

REX GEPFERT

PLANT MANAGER

4211 ENGLE ROAD

FT WAYNE, IN 46804

$219432-4214$

FIELD SERVICES CORP

GLENN R KERLER

PRESIDENT

$P$ BOX 413

CONIFER, CO 80433

$303838-2442$

FINA OIL \& CHEMICAL COMPANY JOHN F BREHER JR MANAGER CONST E HAINT P O BOX 2159

DALLAS. TX 75221

$214750-2642$

FISCA OIL CO INC

WILBERT G CHAMBERS

P $80 X 3363$

KANSAS CITY, KS 66103

FI SONS HESTERN CORPCRATION

MARKETING/SALES DEPT

500-1212 HEST BROADHAY

VANCOUYER $B C$

$604873-3201 \times 207$

FLEXIBLE PACKAGING ASSOCIATION SHERON E WEARY DIRECTOR TECHNOLOGY AND OATA 1090 VERMONT AVE NH

YASHING TON, DC 20005

$202842-3880$

FLORIDA INDUSTRIAL SCALE CO DON P COOK

OWNER

1540 NORTH ST

LONGWOOD:FL 32750

FOOD \& DRUG LAW INSTITUTE

GARY L YINGLING

PRESIDENT

$1701 \mathrm{~K}$ ST NH SUITE 904

WASHINGTON, DC 20006

$202833-1601$

FOOD CHEMICAL NEHS INC

RAYMOND GALANT

MANAGING EDITOR

LIOI PENNSYLVANIA AVE SE

WASHINGTON, DC 20003

$202544-1980$

FOOD MARKETING INSTITUTE

ROBERT O ADERS

PRESIDENT

$1750 \mathrm{~K}$ STREET NH

HA SHING TON, DC 20006

$202452-8444$
FORT WORTH GRAIN EXCHANGE

ALBERT J SILHAVY

2707 DECATUR AVE PO BOX 4422

FORT WORTH, TX 76106

$317626-1071$

FRANKLEN EQUIPHENT INC

RONGNER BERGHARK

PRESIDENT

6643 W $63 R$ D ST

CHICAGO. IL 60638

$312586-7881$

FRANKLIN CHEHICAL INDUSTRIES HILLIAM D SPOHN

DIRECTOR MFG SERVICES

2020 BRUCK ST

COLUMBUS: OH 43207

FRANKLIN ELECTRIC

JOHN H YOUNG

PRODUCT/DISTRI BUTION MANAGER

PO BOX 666

LEV ITTOWN PA 19058

FRANKLIN ELECTRIC CO

BRADFORD MOLNAR

DES IGN ENG INEER

RT 2 BOX 1

WHI TEVILLE, NC 28472

FRANKLIN ELECTRIC CO PACK/WHG ARTHUR L REENSTRA

DIR OF ENGINEERING/MANUF DIV PO BOX 666

LEVITTRWN FA 19058

FRANKLIN INTERNATIONAL

ROBERT W APKING

MFG SER VICES MANAGER

2020 BRUCK ST

COLUMBUS OH 43207

$614445-1404$

FRAZIER PRECISION INSTRUMENT

TUM SCRI VENER

VICE PRESIDENT

16761 OAKMONT AVE

GAITHERSBURG, MD 20877

FREU STEIN LABORATORIES INC

TOM BARNETT

DIRECTOR RESEARCH \& DEVELOPMEN

I 21 NORTH FOURTH ST

ATCHISON, KS 66002

$913367-3945$

FRIGO CHEESE CORPORATION

MAX SHA RE

TECHNICAL DIRECTOR

$P$ P BOX 158

LENA. WI 54139

$414829-5251$

FUEL MERCHANTS ASSOCIATION NJ GEORGE RIEMANN

TECHNICAL DIRECTOR

66 MORRIS AVE

SPRINGFI ELD, NJ 07081

201 379-1100

$G S E$ INC

TOM A UL ICNY

SCALE OPERATIONS MANAGER

23640 RESEARCH DR

FARMINGTON HILLS, MI 48024

$313476-7875 \times 225$ 
G I MICHELLI CO INC

SALES MANAGER

628 HES TER AVE

RIVER RIDGE, LA 70123
$504885-9710$

G T MICHELLI CO INC

TROY E HERR ING

BRANCH MANAGER

425 GUIDICI STREET

JACKSON, MS 39204

$601352-8809$

G T MICHELLI CO INC G T MICHELLI JR

PRESIDENT

10116 HYOE PLACE

RIVER RIOGE: LA 70123
$504885-9710$

G T MICHELLI CO INC

JOSEPH W OVELLA

SERVICE MANAGER

P O BOX 1045

HETAIRIE LA 70004

$504885-9710$

GARNAC GRAIN CO INC

HAL HUDGINS

$P$ C BOX 59

KANSAS CITY, MO 64141

$816932-0140$

GARNAC GRAIN CO INC

WICK W VAN EYCK

VICE PRESIDENT ENGINEER ING

8000 WE ST 110 TH STREET

OVERLAND PARK, KS 66210

$913661-6100$

GARSITE PRODUCTS INC

PETER J HLAVAC

SALES MANAGER

10 GRAND BLVD P $080 \times 289$

DEER PARK NY 11729

GASCO I NC

RI CHARO LOO

VP ENGINEERING \& CONSTRUCTION

PO BOX 3379

HONOLULU. HI 96842

$808547-3570$

GASTOWN

8 ILL D: NEIL

23660 MILES AVE

BEDFDRD HEIGHTS, OH 44128

$216475-9212$

GENERAL DYNAMICS CONVAIR DIV

URBAN I SWEENEY

CHIEF LIBRAR IAN

P BOX 85386

SAN DIEGO. CA 92138

$619573-4876 \times 1073$

GENERAL ELECTRO OYNAMICS CORP

KEVIN C BEGLEY

CUSTOMER SERVICE

P O BOX 15008

ARLINGTON, TX 76015

GENERAL ELECTRONIC SYSTEMS INC W GARY OOLL

VICE PRESIDENT-MARKETING

$914 \mathrm{SE} 14 \mathrm{TH}$ PLACE

CAPE CORAL. FL 33904

$813574-2313$
GENERAL FOODS CORPORATION

HARVEY A HALZER

MANAGER CORP QUAL ITY ASSURANCE

250 NORTH ST

WHITE PLAINS. NY 10625

$914335-5515$

GENER AL METER SERVICE INC

RONALD C YOUNG

PRESIOENT

123 H TH $80 \times 1$

RUSSELL. KS 67665

$913483-3758$

GENERAL MILLS INC

ROBERT L NELSON

MGR PHYSICAL TESTING-8AKING SE 9000 PLYMOUTH AVE $N$

MINNEAPOL IS: MN 55427

$612540-2729$

GENERAL MILLS INC SPERRY DIV WILLIAM C MAILHOT

DIRECTOR OUALITY CONTROL

9200 WAYZATA BLVO

MINNEAPOLIS. MN 55426

$612540-2354$

GEO A HORMEL \& CO

JAMES E WESTRICK

OC ENGINEER

$B 0 X 800$

AUSTIN. MN 55912

$507437-5754$

GEORGE A LINTON INC

GEORGE A LINTON

CONSULTANT

1808 CANTON ROAO

BIRMINGHAM. AL 35216

205 B23-2855

GERBER PRODUCTS CO

RAY E VANHUSS

DIRECTIR GOVERNMENT RELATIONS

445 STATE STREET

FREMONT. MI 49412

$616928-2267$

GIANT FOOO INC

DAVID M RICHMAN

OIRECTOR OF OUALITY ASSURANCE

PO $80 \times 1804$

WASHINGTON, DC 20013

$301341-4134$

GILBARCO INC

ART KROLL

MANAGER REGULATORY AFFAIRS

7300 W FRIENOLY AVE

GREENSBORO. NC 27420

$919292-3011 \times 255$

GILBARCO INC

CLAUDE R PARENT

OIRECTOR-NATIONAL ACCOUNTS

7 EAS TWOOO DR

ORINDA. CA 94563

$415 \quad 376-5697$

GILMARCO INC

RICHARD L WHIPPLE

REGUL AIURY ENGINEER

3511 W MARKET ST POB 22087

GRFFNSBORO. NC 27420

919 292-3011

GIRI BROTHERS

T SF SHAGIR I

PARTNER

5I RAJAJI SALAI MADRAS 600001

MADRAS TAMIL 
GOLDEN GRAIN MACARONI CO

DENNIS T OEDOMENICO

SECRETARY-TREASURER

SAN LEANDRO, CA 94578

$415357-8400 \times 324$

GOLDEN RECYCLE CO

LARRY K HOPKINS

ELECTRI CAL ENGINEER

3000 YOUNGFIELD SUITE 230

$\angle A K E$ HOOD, CO 80215

$303277-5794$

GOLDEN RECYCLE COMP ANY

DAVID M ODAY

NATIONAL SALES MANAGER

3000 YOUNGFIELD ST SUITE 230

LAKEWOOD CO 80215

303 277-5771

GOODMARK FOODS INC

C H ISLEY

DIRECTOR QUALITY ASSURANCE

$P$ O BOX 187

GARNER, NC 27529

$919772-1511$

GRAIN ELEVATOR \& PROCESSING

JOHN J HEALY

EXECUTIVE VICE PRESIDENT MINNEAPOLIS, MN 554150026

$612339-4625$

GRAND TRUNK WESTERN RAILROAD

NARENDRA S PATEL

BRIDGE \& STRUCTURE ENG INEER

131 LAFAYETTE RM 903

DETROIT MI M 48226

GREAT NORTHERN PAPER COMPANY BR I AN HAML IN

MASTER JOURNEYMAN

$P$ O BOX 240 WEST BRANCH SHOP

MILLINDCKET, ME 04462

$207723-5 i 3 i \times 1435$

GREATER NY METRO FOODS

DONALD $R$ WALL

275 MADISON AVE

NEW YORK. NY 10016

$212689-8600$

GREATER WASHINGTON MARYLAND

ROY E LITTLEFIELD

9420 ANNAPOLIS RD SUITE 307

LANHAM, MD 20706

$301577-2875$

GROCERY MANUFACTURERS OF AMER

MAHLON A BURNETTE III

PUBLIC AFFAIRS CONSULTANT

631 WALKER ROAD

GREAT FALLS, VA 22066

$703759-5984$

GROCERY MANUFACTURERS OF AMER

ANDRE W M MORE

SCIENCE ASSOCIATE

IOIO WISCONSIN AV NH STE 800

WASHINGTON. DC 20007

$202337-9400$

$H \varepsilon S$ ENTERPRISES

W DOOLEY

GENERAL MANAGER

P O BOX 1050

FARMINGTON, NM 87499
H J HEINZ CO

SIRGINIA M LEY PO BOX 57

PITTSBURGH, PA 15230

$412237-5214$

H P HOOD INC

HARRY L WILDASIN PHD

CONSULTANT GOVT RELATIONS

500 RUTHERFORD AVE

BOSTON, MA O2129

HAL VER SON COMPANY

GEORGE MAJORS

MANAGER

235 HEST PAXTON AVE

SALT LAKE, UT 84101

$801467-9423$

HAMMEL SCALE COMPANY INC

JOHN R HAMMEL

$1545 \mathrm{~N}$ MOSLEY

WICHI TA, KS 67214

$316264-1358$

HAMMEL SCALE OF KC INC

RALPH TAYLOR

SER VICE SUPERVISOR

8421 NIEMAN ROAD

LENEXA. KS 66214

$913492-1807$

HARDY INSTRUMENTS INC

HUGH W NESS

PRESIDENT

3565 CORPORATE COURT

SAN DIEGO, CA 92123

$619565-7701 \times 446$

\author{
HAROLD WAINESS $E$ ASSOCIATES \\ HAROLD WAINESS \\ OMNER/CONSULTANT \\ 464 CENTRAL \\ NORTHFIELD, IL 60093 \\ $312446-2402$ \\ HARRIS ASSOCIATES \\ HAROLD E HARRIS \\ PRE SIDENT \\ 22R TOWN \& COUNTRY \\ HOUSTON. TX 77024 \\ $713465-8034$ \\ HEINZ USA \\ $J A M E S$ G FOX \\ PITTSBURGH, PA 15230
}

HEINZ USA DIV OF H J HEINZ CO JOHN S ELLIOTT JR

1062 PROGRESS ST

PITTSBURGH. PA 15212

$412237-5862$

HELENF CURTIS INDUSTRIES INC MARVIN SIROTA

CORPORATE DIRECTOR MANUFACTURE

4401 W NOR TH AVE

CHICAGO. IL 60639

$312292-2121$

HERMAN AND ASSOCIATES

MAR ILYN J HERMAN

PRE SIDEN T

$2300 \mathrm{M}$ ST NW SUITE 800

WASHINGTON, DC 20037 
HERSHEY FOODS CORPORATION

VINCENT J WATTAI

OUALITY AS SURANCE SUPERVISOR

19 E CHOCOLATE AVE

HER SHEY, PA 17033

$717534-4686 \times 4686$

HI-SPEED CHECKWEIGHER CO INC VICTOR DEL ROSSO

PRESIDENT

605 WEST STATE ST

ITHACA. NY 14850

$607273-5704$

HILLS PET PRODUCTS

RONALD E CROH

QA MANAGER

$80 \times 148$

TOPEKA KS 66603

$913354-8340 \times 38$

HOBART CORP

FRED H KAT TERHE INRICH

MANAGER AGENCY/DESI GN POLICY TROY OH 45374

$513332-2037$

HOBART CORP

JACK SHEEHAN

MANAGER

28 BY PASS BOX 957

MANCHESTER NH 03105

$603623-3622$

HOBART CORPORATION

EUWIN E BOSHINSKI

DIR ADVANCED DEVELOPMENI

WORLD HEADOUARTERS

TROY, OH 45374

$513332-2637$

HOBART CORPORATION

GERALD W DAVIS

SER VICE MANAGER

10631 SUMMIT ST

LENEXA: KS 66215

$913469-9600$

HOBART CORPORATION

JAMES R GARRETT

MANAGER PRODUCT ENGINEERING

TROY, OH 45374

$513 \quad 332-3000$

HOBART CORPORATION

ROBERT E JONES

NATIONAL ACCOUNTS REPR

$55 B 4$ BANDINI BLVD

BELL? CA 90201

$213260-7321$

HOBART SALES \& SERVICE

HAROLD L HOLDORF

$A G E N T$

2116 CLEVELAND AVE

ANCHORAGE AK 99503

907 248-0700

HOFFER FLOW CONTROLS INC

RICHARD L GRUSKDS

$\checkmark I C E$ PRESIDENT/ENGINEER ING

P O BOX 130

PORT MONMOUTH, NJ 07758

$201787-1997$

HOFFER FLOW CONTROLS INC

KENNETH R HOFFER

PRESIDENT

$P$ O BOX 130

PORT MONMOUTH, NJ 07758

$201787-1997$
HOLBROOK JONSON BRESSLER ET AL $T$ IMOTHY $R$ EVANS

ATTORNEY

315 S MONUMENT AVE

HAMILTON, OH 45011

$513868-7600$

HOLLY SUGAR CORPORATION

RICHARU S PARR ILL

DIRECTOR OF CUSTOMER SFRVICES

$P$ O BOX 1052

COLORADD SPRINGS, CO 80901

$303471-0123 \times 208$

HOL TGREVEN SCALE \& ELECT CORP CHARLES I HOLTGREVEN

PRES IDENT

420 EAST LINCOLN ST

FINDLAY, OH 45840

HOOKER CHEMICALS \& PLASTICS CO TA LESNIOWSKI SUPERVISOR PLANT TRAFFIC $P O B O X 159$

NIAGARA FALLS. NY 14302

$716278-7622$

HORNER EOUIP CO

TOM M OKON

PRESIDENT

$400 N$ I 14 TH ST $P$ O BOX 1288

SAGINAW. MI 48606

$517755-5327$

HOT I INGER BALDWIN MEASUREMENTS HARRY E LOCKERY

139 NEWBURY ST

FRAMINGHAM, MA 0170

$617875-8282$

HOWE RICHARDSON

JOHN W AOUADRO

VICE PRESIDENT INDUSTRIAL OIV

680 VAN HOUTEN AVE

CLIFTON. NJ 07015

$201471-3400 \times 243$

HOWE RICHARDSUN SCALE COMPANY JOHN MACDONALD

$D I R E C T O R$ OF ENGINEERING

680 VAN HOUTEN AVENUF.

CLIFTON. NJ 07015

$201471-3400$

HUMKO PRODUCTS KRAFT CORP

DOWARD T BENEF IELD

QUAL IIY CONTROL MANAGER

P 0 BOX 398

MEMPHIS, TN 38101

HUNTOON EOUIPMENT CO

ROBERT M FREEBURG

PRES

624 LIVERNDIS

FERNDALE. MI 48220

$313543-6900$

HUSKY C.ORPORAT ION

GRENVILLE G SUTCLIFFF

I DAILEY INDUSTRIAL PARK

PACIFIC, MO 63069

$314257-3073$

HYPONEX CORPORATION

JOHN E SOLTES

DIREC IOR OF PACKAG ING

4501 CIRCLE 75 PKWY STE R2250

ATLANTA. GA 30339

$404955-5910$ 
I BM

JOHN PR OVETERO

PLANNER

G68/B602 PO BOX 12195

RESEARCH TRI ANGLE, NC 27709

$919836-5456$

ICE CREAM MNFRS ASSN

DAHN M BRYDON

DIRECTOR PUBLIC AFFAIRS

$88816 \mathrm{TH}$ ST $\mathrm{NH}$

WASHINGTON: DC 20006

202 296-4250

ILLINOIS CENTRAL GULF RAILROAD LAYERN H CARROLL

CHIEF SCALE INSPECTOR

600 GILMORE ST

CENTRALIA: IL 62801
$618533-3319$

ILLINOIS DEPT OF AGRICULTURE BOB SCHWARBERG

BUREAU CHIEF

801 EAST SANGAMON AVENUE

SPRINGFIELD: IL 62706

IMPERIAL SUGAR CO

JOE E RUZICKA

PACKAGING SYSTEMS ENGINEER

SUGAR LAND: TX 77478

INDEPENDENT LUBRICANT MFR ASSN

NANCY J DEMARCO

EXECUTIVE DIRECTOR

1055 THOMAS JEFFERSON NH $\$ 302$

WASHING TON DC 20007

INDIANA OIL MARKETERS ASSN

L JACK STOUGH

EXECUTI VE DIRECTOR

8780 PURDUE ROAD

IND I ANAPOL IS. IN 46268

$317875-9057$

INDUSIRIAL SCALE SERVICE

LINDSEY L EPPERSON

GEN MGR

RT 2 BOX 355

MARSHALL TX 75670

$214935-3027$

INDUSTR IAL SCALES INC

ROBERT M HAMILTON

PRESIDENT

RTE 5 BOX 99B I-55 SOUTH

JACKSON, MS 39212

$601372-5384 \times 270$

INFORMATION RESOURCES INC

JAMES E PEEPLES

DIRECTOR LEGISLATIVE AFFAIRS

499 S CAPITOL ST SUITE 406

WASHING TON, DC 20003

$202554-0614$

INLAND NORTH WEST SCALE CO

JACK B BRADLEY

PRESIDENT

PO BOX 113355602 E DE SMET

SPOKANE , HA 99211

$509535-4295 \times 323$

INLAND NORTHWEST SCALE CO INC AL I CE W LACY

PO BOX 11355 PARKWATER STA

SPOKANE, HA 99211
INST CENTRO AM INV TEC IND

FRANCISCO AGUIRRE

BIOCHEMIST

AVENIDA LA REFORMA 4-47ZONA 10

GUATEMALA GUATEMALA 310631

INSTITUTE FOR INDUSTRIAL $R \& S$ MART IN A HYNES

HEAD-METROLOGY LABORATORY

BALLYMUN ROAD

DUBLIN 9

INT BUREAU OF LEGAL METROLOGY $Z$ REFER OWSKI

ASS ISTANT DIRECTOR

11 RUE TUR GOT

75009 PARIS

INT SOC OF WEIGHING \& MEAS

A H ENGLE

EXECUTIVE DIRECTOR

2506 GROSS POINT RO

EVANSTON IL 60201

INT ERAMER ICANO DE METROLOGIA

RAFAEL STE INBERG

PRESIDENTE SISTEMA

SIM-INTI

1650 SAN

7525213

INT ERCOMP COMPANY

ROBER T E KROLL

2520 XEN IUM LANE

PLYMOUTH. MN 55441

$612559-0646$

INTERNATIONAL ROAD DYNAMICS

SHARON PARKER

OFFICE MANAGER

211-3502 TAYLOR ST F

SASK SASKATCHEHAN

INTERSTATE MILK PRODUCERS COOP WILLIAM B HASTINGS

MGR OUALITY \& FIELD SERVICES

$P O B O X 127$

SOUTHAMPION, PA 18966

$215322-0200$

INTL ASSN ICE CREAM MFG

GLENN P WITTE

VICE PRES IDENT

888 16TH ST NH

WASHINGTON, DC 20006

IRWIN MEASURING TOOL CO ROBERT W CARTER

$P$ BOX 471

PATCHOGUE, NY 11772

$516 \quad 289-0500$

J $B$ DEE \& CO INC

STEPHAN A

$1722 \mathrm{~W} 16 \mathrm{TH}$ ST

IND IANAPOLIS. IN 46202

$317635-5548$

$J$ L PRESCOJT CO

DON AYERS

MANAGER OUALITY CONTROL

27 EIGHTH ST

PASSAIC. NJ 07055

$201777-4200$ 
$\checkmark$ L PRE SCOTT COMPANY

HOWARD ALBOUM

27 EIGH ST P 0 BOX 778

$201777-420007055$

$J$ M SMUCKER CO

M L LAZEAR JR

MANAGER REGULATORY AFFAIRS

STRAWBERRY LANE

ORRVILLE: OH 44667
$216 \quad 682-0015$

J P STEVENS CO INC

DIRECTOR CUSTOMER-SUPPLIER AFF

P O BOX 2850

GREENVILLE: SC 29602

$516 \quad 349-9082$

$J$ RED GASKINS \& CO

CARLTON J GASKINS

OWNER

HWAY 52 SOUTH
LAKE CITY SC 29560
$803394-8830$

JAMAICAN BUREAU OF STANDARDS TWEEDSMUIR C MITCHELL

STANDARDS SCIENT IFIC OFFICER 6 WINCHESTER ROAD KINGSTON 10 KINGSTON

$8099263140 \times 217$

JAMES RIVER CORPORATION

RICHARD L DAVIS

ADMINISTRATOR REGULATORY COMPL

1915 MARATHON AVE

NEENAH, WI 54956

$414729-8174$

JAPAN MEASURING INSTRUMENT FED KE SAKATSU KO I ZUMI

MANAGING DIRECTOR

25-I NANDO-CHO SHIN JUKU-KU

TOKYO

JBBRADY INCORPORATED

JOHN B BRADY

PRES IDENT

81 I AL VORD ST

SYRACUSE, NY 13208

$315422-9271$

JOHN CHATILLON \& SONS INC

$J$ FRITS NIEMOLLER

PRESIDENT

$8330 \mathrm{KEW}$ GARDENS RD

KE GARDENS. NY 11415

$212847-5000 \times 12$

JOHN J MCINTYRE SONS INC

JOHN J MCINTYRE

PRESIDENT

514 KNORR

PHILADELPHIA, PA 19111

$215745-3304$

JOHN MORRELL

DON J KINDT

SCALE REPAIR SUPERVISOR

1400 NORTHWEBER PO BOX 1266

SIOUX FALLS. SD 57117

$605338-8200 \times 223$

JOHNSON SCALE CO

WAYNE $H$ JOHNSON

$P$ O BOX 1329

CALDWELL. NJ 07006
KAL KAN FOODS INC

MAN MCKNIGHT

MANAGER TECHNICAL SERVICES

3376 E 44TH STREET

VERNON, CA 90058

$213587-2727$

KAMLAR CORP

RICHARD C SEALE

PRESIDENT

105 KAMLAR DRIVE

ROCKY MOUNT: NC 27801

919 443-2576

KANAWHA SCALES \& SYSTEHS

WILLI AM J MCHALE

$\checkmark$ ICE PRESI DENT

ROCK BRANCH INDUSTRIAL PARK POCA, WV 25159

$304755-8321$

KEEBLER COMPANY

JACK A R ISER

MANAGER LABORATORY SERVICES

ONE HOLLOW TREE LAHE

ELMHURST IL 60126

$312833-2900$

KELLOGG COMPANY

$M W$ SCOT

USFPO OUAL ITY PROGRAMS

BAT TLE CREEK. MI 49016

KELLY OIL COMPANY

MICHAEL FOSTER

REG IS TER ED SERVICEMAN

$B O X 1016$

RAWLINS, WY 82301

$307324-3332$

KENOSHA CITY HEALIH DEP

GEORGE A $Z$ IMMER $R$

SUPERVISOR ENVIRONMENTAL MLTH $62552 N D$ ST

KENOSHA. HI 53140

$414656-8170$

KERR MC GEE COAL CORPORATION H E SCHUMACHER

DIRECTOR SALES \& SERVICE

KERR MC GEE CIR PO BOX 25861

OKL AHOMA CITY. OK 73125

$405270-3735$

KESON INDUSTRIES INC

FRANK J NOSEK

$\checkmark I C E$ PRESIDENT

28 W 715 MOUNT ST

WARRENVILLE. IL 60555

$312393-3232$

KIKKOMAN FOODS INC

MILTON E NESHEK

ASST SECY $E$ GEN COUNSEL

P 0 BDX 291

ELKHORN, WI 53121

$414723-3220$

KIPPENBROCK SCALE SERVICE

JOSEPH 8 KIPPENBROCK

2408 PLEASURE HOUSE RD

VIRGINIA BEACH. VA 23455

KITCHENS OF SARA LFE

JAMES CULLEN

SCALE TECHNICIAN

500 WAIJKEGAN RD

DEERF IFLD, IL 60015

$312945-2525 \times 2282$ 
KIUI BRANDS INC PETER A BURKE LABORATORY MANAGER

ROUTE 662 NORTH

DOUGLASSVILLE, PA 19518

$215385-3041$

KLEESPIE TANK \& PETROLEUM EQ BOB KLEESPIE

OHNER

$P$ O BOX 106

MORRIS. MN 56267

$612589-2100$

KNUDSEN CORP

JOSEPH G HECK PHD

OUALITY ASSURANCE

1900 WEST SLAUSON A VE

LOS ANGELES: CA 90047

$213744-7000$

KNUDSEN CORP

DOUGLAS D MELOTT

QUALITY CONTROL MANAGER

415 KANSAS AVE/PO BOX 5018

MODESTO. CA 95352

$209575-5100 \times 5123$

KOLMAR LABORATORIES INC

KEITH A BURDICK

QUALITY ASSURANCE MANAGER

$P$ O BOX 1986

DENTON, TX 76201

$414271-3131 \times 260$

KOPPENS / SCHLUMBER GER

RICHARD P TRASK

WTS E MEASURES LIAI SON

181 STETSON RD

NOR WELL, MA 02061

$617849-1368$

KRAFT I NC

SHARON L IRVINE

ASSOC ATTORNEY ADY/FOOD \& DRUG GLENVIEW IL 60025

$312998-2796$

KRAFT INC

DARREL D MOATES

STATISTICAL TECHNOLOGY MANAGER

KRAFT COURT

GLENVIEW: 1 IL 60025

KRAFT INC

ELLEN THOMAS

MANAGER REGULATORY COMPLIANCE

KRAFT COURT

GLENVIEW. IL 60025

KROGER CO

DA VID P LEAHY

TECHNICAL CONSULTANT

2 CAMPBELL DRIVE

HIGHLAND HEIGHTS, KY 41076

$606572-2200$

KROGER CO DAIRY

JOHN C WURGLER

MANAGER OUALITY ASSURANCE

400 S SHORTRIDGE RD

INDI ANAPOLIS. IN 46219

$317356-6800 \times 14$

KROGER COMPANY

STU ROE MKE

2 CAMPBELL DR

HIGHLAND HEIGHTS, KY 41076

$607572-2223$
KUBOTA AMERICA CORPORATION

YOSHI YUKI WAKI SAKA

$R$ AND D MGR

CHRYSLER BLDG 405 LEXINGTON AV

NEW YORK. NY 10174

$212 \quad 490-8050$

LAB BAL ANCE E HICROSCOPE SVC ROGER CRAWFORD

OWNER

203 SCENIC DR

OAK RIDGE: TN 37830

$615 \quad 483-9652$

$\angle A N C E$ INC

ROBERT S CARLES

ATTORNEY

PO BOX 32368

CHARLOTTE NC 28232

(5) 5421

LAND O LAKES INC

DOUGLAS R ENGEBRETSON

DIR QUALITY ASSURANCE \& TECHN P O BOX II 6

MINNEAPOLIS, MN 55440

$612331-6330 \times 390$

LAND 0 LAKES INC

JOEL SIANGELAND

MANAGER CORPORATE OUALITY ASSU

$P$ O BOX 116

MINNEAPOLIS, MN 55440

$612481-2281 \times 379$

LANDVATER ASSOCIATES

JOHN H LANDVATER

PRESIDENT

$P$ D BOX 654

SUMMII. NJ 07901

$201273-8757$

$\angle A N E \&$ ANDERSON INC

ROGER LANE

7479 LEMHI ST

BOISE. ID 83709

$208376-4300$

LAWRYS FOOD INC

ERNIE H YASUKDCHI

O A MANAGER

570 WEST AVENUE 26

LGS ANGELES. CA 90065

$213 \quad 225-2491$

LEHN \& FINK PROD/STERLING DRUG RANDALL L STRUBHAR

MANAGER OUALITY CONTROL

316 N LIMIT ST

LINCOLN. IL 62656

$217735-1555 \times 231$

LEVER BROTHERS COMPANY

EDWARD M SULL IVAN

OUALITY ASSURANCE MANAGER

5300 HOL AB IRD AVE

BAL TIMORE. MD 21224

$301342-2100 \times 322$

LIBERTY GLASS CO

DON E BOL IN

MANAGER OF OUALITY CONTROL

PO BOX 520

SAPULPA, OK 74066

$918 \quad 224-1440 \times 244$

LIOUID CONTROLS CORPORATION

MELVIN C HANKEL

CHIEF DESIGN ENGINEER

WACKER PARK

NOR IH CHICAGO, IL 60064

$312689-2400$ 
LIOUID TRANSFER SVS LTD

G I AN PATTERSON

P ENG

1325 SHAWSON DR/MISSISSAUGA

ONTARIO L4H

$416678-6314$

LOCKHEEO ELECTRONICS CO

JOSEPH F DEVIIT

SER VICE MANAGER

US HWY 22

PLAINFIELD. NJ 07061

$201757-1600 \times 2723$

LOCKHEED MISSILES \& SPACE CO J M SUR ACI MANAGER CAL IBRATION LABORATORY PO BOX 6429

BREMERTON。 WA 98315

206 396-6772

LOCKHEED MISSILES \& SPACE CO

ROBERT $L$ WEBER

MANAGER METROLOGY

P D BOX 504 BLDG 195A 0/48-70

SUNNYVALE。 CA 94086

$408742-2957$

LOOEC INC

DOUGLAS G BRDOKS

PRESIDENT

PO DRAWER D

LYNNWOOD. WA 980461300

$206775-6471$

LODEC INC

ROBERT B HOOD

DIRECTOR OF ENGINEERING

DRAWER D

LYNWOOD. WA 98036

$206775-6471$

LOUIS DREYFUS CORPORATION

GERRY KRUEGER

24 RICHMOND HILL AVE

$203357-8444$

LOUISIANA DEPT OF TRANS \& DEV JARVIS J POCHE

ASST MATER IALS ENGINEER

PO BOX 44245 CAPITOL STATION

BATON ROUGE, LA 70804

$504925-6722$

LUDENS INC

LEONARO S CHANOLER

QUALITY CONTROL MANAGER

200 NOR TH 8 TH ST

READING, PA 19603

$215376-2981$

LUFKIN DIV COOPER IND

FRED E REIKOWSKY

QUALIIY CONTROL MANAGER

$B 0 \times 728$

APEX, NC 27502

$919362-7511 \times 636$

M \& M MARS

ANTHONY HOOO

QUALITY SYSTEMS SPECIALIST

HIGH ST

HACKETTSTOWN, NJ 07840

$201850-2257^{\circ}$

MAIN AVE

SY FEINLAND

PITNEY BOWES INC

NORWALK,$C T$ T 06851

$203853-7113$
MAPCO INC

WAYNE A LATIMER

SENIOR MEASURE MENT ENG INEER

$1800 \mathrm{~S}$ BALTIMORE ST

TULSA, OK 74101

$918599-3635 \times 635$

MARATHON PETROLEUM CO

ROBERT J HARRI NGTON

MGR SAFETY SECURITY MKTG OPS

539 SOUTH MAIN ST

FINDLAY. OH 45840

$419422-2121$

MARATON OIL CO

MICHAEL I SCHAIBLE

SPECIAL REPRESENTATIVE

539 MAIN ST

FINOLAY. OH 45840

$419422-2121$

MAR ION COUNTY AUDITORS OFFICE WAYNE N MAHONEY

I NSPECTOR

CDURTHOUSE ROOM 5

MARION. OH 43302

$614387-5871$

MARKETING SPECIAL PROJECTS THOMAS L MORROW

MANAGER

19250 VAN NESS AVE

TORRANCE. CA 90501

$213320-8900 \times 67$

MARTIN DECKER COMPANY

THOMAS G GORDON

QUALITY ASSURANCE MANAGER

1200 CYPRESS CREEK ROAD

CEDAR PARK. TX 78613

$512331-0411 \times 265$

MAR T IN-DECKER

JOHN E GARNSEY

GULF COAST REGIDNAL SALES MGR

2630 FOUNT AINVIEW SUITE 410 HOUSTON. TX 77057

$713780-8787$

MAR T IN-DECKER

WAL TER J GOULET

SENIOR REGIONAL SALES

4989 TURTLE ROCK DR

MARIETTA, GA 30066

$404928-7051$

MARYLAND PUMP TANK $\&$ ELECTRIC FRANK $X$ SINGLETON

PRE S IDEN T

2512 ERICK STREET

BAL IIMORE, MO 21230

$301837-0770$

MASCOTT EQUIPMENT COMPANY INC WILLIAM J MASCOTT PRESI DENT

435 NE HANCOCK ST

POR TLANO, OR 97212

$503282-2587$

MASSTRON SCALE INC

BEN DILLON

PRESIDENT

6600 HUNTLEY ROAD

COLUMBUS, OH 43229

$614436-3292$

MASTER BUILDERS

JAMES C MARAS

QUALITY ASSURANCE

23700 CHAGRIN BLVD

CLEVELAND, OH 44122 
MASTER SCALES

GARY R MCDONALD

713 HWY 82 HEST

GREENHOOD MS 38930

$901346-4619$

MC CADAM CHEESE CO INC

HILLIAM F HARRIGAN

PLANT MANAGER

12 ANNETTE

HEUVELTON: NY 13654

$315344-2441$

MCCORHICK \& CO

MARK MORE

PACKAGING $\&$ PRODUCT CDMPLIANCE

11350 MCCORMICK ROAD

HUNT VALLEY, MD 21031

$301667-7442$

MCCORMICK CO

RONALD F FLEMING

TECHNICAL SERVICE MANAGER

1311 SCHILLING PLACE

SAL INUS. CA 93912

$408758-2411 \times 221$

MCKESSON CORP LAW DEPARTMENT

ALFRED E JOHANSON

SENIOR COUNSEL

NEW YORK NY 10017

$212573-0332 \times 330$

MEASUREGRAPH CO

GLEN MAYS

MÁNAGER OF SERYICE

1694 LARKIN WILLIAMS ROAD

FENTON: MO 63026

MEA SUREMENT AND CONTROL

MIL TON H ARONSON

EOI TOR PUBL ISHER

2994 LIBERTY AYE

PITTSBURGH. PA 15216

$412343-9666$

MEA SUREMENT SYSTEMS INTERNTL

DAVID C ENGLISH

PRE SIDENT

12622 INTERURBAN AVE S

SEATTLE, WA 98168

$206767-7433$

MEENAN OIL CO INC

CHARLES C SHANNON

OPERATI ONS MANAGER

$P$ O BOX 67

LEVITTOWN, PA 19059

MEISSNER TISCH \& KLEINBERG

HOWARD B TISCH

PARINER

275 MADISON AVE

NEW YORK, NY 10016

MENNEN COMPANY

WILLIAM V STANSKY

O A INSPECTION MANAGER

MORRISTOWN. NJ 07960

$20163 i-9095$

MER ICO INC

GEORGE WEBER

CARROLLTON. TX 75006
MERRICK SCALE MFG COMPANY

LOUIS C DIANI

SER ICE DEPARTMENT

MERR ICK DR IVE

ROSELAND, NJ 07068

201 226-0656

METERS INC

A H DUNCAN

HWY 205 \& VICTORIA ROAD

WOODSTOCK, GA 30188
$404926-2917$

METRO EQUI PMENT CORPORATION

JACOUES C

1235 REAMWOOD AVE

SUNNYVALE, CA 94086

$408734-5400$

METROLOGY GALLIED TECHNOLOGIES

PHILLIP A PAINCHAUD

CONSULTANT

1110 WOROTHY DR

BREA. CA 92621

$714529-6604$

METROLOGY SERVICES CORP

LLOYD CARVER

P O BOX 7029

HUNTSVILLE:AL 35805

METTLER INSTRUMENT CORP

WALTER HAUSHERR

DIR MGMT SERVICES

BOX 71

HIGHTSTOWN. NJ 08520

$609448-3000$

METTLER INSTRUMENT CORP

WAL TER E KUPPER

MANAGER ENGINEERING

$B O X 71$

HIGHTSTOWN, NJ 08520
$609448-3000$

METTLER INSTRUMENT CORPORATION WOL FGANG LEMME

MANAGER TECHNICAL SERVICES

PRINCFTON-HIGHTSTOWN ROAD

HIGHTSTOHN. NJ 08520

$609448-3000 \times 221$

METTLER INSTRUMENTE AG

HANS EIGENMANN

TECHNICAL SALES

$\mathrm{BOX} 71$

HIGHTSTOWN. NJ 08520

$609448-3000 \times 254$

MICHIANA SCALE COMPANY

VIC W STEINHOFFER SR

OWNER

$1524 M 139$

BENTON HARBOR. MI 49022

$616927-1471$

MICHIGAN PEAT CO

GARY WYSE

DISTRIBUTION MANAGER

2243 MILFORD ST

HOUSTON. IX 77098

$713522-0711$

MICHIGAN PETROLEUM ASSOCIATION

IHOMAS G CORDELL

EXECUTIVE DIRECTOR

1200 MICHIGAN NATIDNAL TOWER

LANSING. MI 48933

$517487-9139$ 
MICRO MOTION

CATHY A APPLE

FLOH SYSTEMS ENG INEER

7070 WINCHESTER CIRCLE

BOULDER, CO $8030 \mathrm{I}$

$303530-8530$

MICRO MOTION INC

JOEL BL UMENTHAL

7070 WI NCHESTER CIRCLE

BOULDER CO 80301

$303530-8400$

MICRO MOTION INC

RANDY JENSON

7070 HINCHESTER CIRCLE

BOULDER CO 80301

$303530-8400$

MICRO MOTION INC

CHUCK MYERS

7070 WINCHESTER CIR CLE

BOULDER . CO 80301

$303530-0530$

HICRO MOTION INC

LEE SMI TH

NEW PRODUCTS MANAGER

7070 WINCHESTER CIR

BOULDER - CO 80301

$303530-0530$

MICRO MOTION INC

KEVIN D WOOD

CAL IBRAT ION SUPERVISOR

7070 WI NCHESTER CIR CLE

BOULDER CO 80301

$303530-8446$

MID MISSOURI SCALE INC

RICHARD L PHILLIPS

PRESIDENT

RR 4 BOX $193 \mathrm{~F}$

SEDALIA, MO 65301

MIDWEST SCALE COMPANY

ROBERT L KENNEDY

PRESIDENT

1327 SEVETH ST

ROCKFORO, IL 61108
$815968-3731$

MIDWESTERN SCALE CO

JAMES BELL

SALES AND INFORMATI ON

1 PECAN PLAZA

NEVADA. MO 64772

$417667-6333$

MILK \& ICE CREAM ASSOCIATIONS

JOHN F SPEER JR

PRESIDENT

88816 TH STREET NH

HASHING TON, DC 20006

MILLER BREHING CO

ROBERT B CORNEY

3939 WEST HIGHLAND BLVD

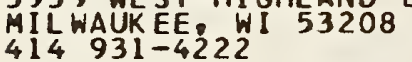

MILLERS NATIONAL FEDERATION

TOM KLEVAY

$\checkmark$ ICE PRESIDENT

600 MAR YLAND AVE STE 305 WEST

WA SHINGTON, DC 20024
$202484-2200$
MINERAL INSULATION MFGRS ASSOC SHELDON H CADY

EXECUTIVE VICE PRESIDENT

382 SPRINGFIELD AVE

SUMMI N NJ 07901

$201277-1550$

MINNESOTA MINING \& MFG

RICHARD H HEBER

METROLOGY LAB

BLDG 544-1-02

ST PAUL, MN 55144

$612733-2674$

MINNETONKA INC

JOHN L NEVEAUX

DIRECTOR REGULATORY SERVICES

JONATHAN INDUSTRIAL CENTER

CHASKA. MN 55318

$612448-4181 \times 260$

MINNKOTA MEIER COMPANY

JAMES F LAMB

OWNER

190 3RD ST NE

WATERTOWN, SD

57201

$605882-2270$

MISSOURI PACIFIC RAILROAD CO EDWARD F PASCHAL

CIVIL ENGINEER

RM $1211210 \mathrm{~N} 13 T H$ STREET

ST LOUIS. MO 63103

31462 ? -2861

MO DEPT OF AGRICULTURE

RON HAYES

PETROLEUM LAB SUPERVISOR

$P$ D BOX 630

JEFFERSON CITY. MO 65102

$314751-2922$

MOBAY CHEMICAL CORP

VERNON L CRAIG

CHIEF INSPECTOR

PO BOX 4913 HAWTHORN ROAD

KANSAS CITY. MO 64120

$816242-2000 \times 2416$

MOBIL OIL CORPORATION

P T OLSON

PLANT ENGINEER ING MANAGER

3225 GALLOWS RD ROOM $2 N$

FAIRFAX, VA 22037

$703849-3730$

MOB ILE INSTRUMENJ CO INC JERRY E GAMOTIS

$7 O 1$ S CONCEPTION ST

MOB ILE, AL 36603

\section{MON SANTO}

H CARL HOEFER JR

PRODUCT MANAGER - F3MG

$800 \mathrm{~N}$ L INDBERGH

ST LOUIS. MO 63166

$314694-5361$

MONSANTO CO

GARY W LINDLEY

PRODUC I ION SUPERVI SOR

$P D$ BOX 473

MUSCATINE. IA 5276 I

319 263-0093 $\times 275$

MONSANTO COMPANY

HARRISUN N HOWE

MATERIAL HANDL ING MANAGER

$800 \mathrm{~N}$ LINDBERGH BL VD

ST LOUIS. MO 63166

$314694-5671$ 
MONSANTO COMPANY (C2NJ)

DAVIDE HOLFERSBERG ER

MANAGER OUALITY ASSURANCE

$800 \mathrm{~N}$ LINDBERGH BLVD

ST LOUIS. MO 63167

$314694-3365$

MONSANTO POLYMER PRODUCTS CO $R$ L SPARKS

GENERAL SUPERINTENDENT TSD NITRO, WV 25143

$304755-3341$

MONTEREY MUSHROOMS

JONATHAN A LIBBY

MANAGER - QUALITY ASSURANCE

$P$ O $B O X 29$

WATSONVILLE, CA 95077

$408728-145 i$ X0242

MONTEREY HUSHROOHS

RAMONA PHILLIPS

OUALITY CONTROL SUPERVISOR

HWY 75 SOUTH P O BOX

MADISONVILLE, TX 77864

$409348-3511$

MONTEREY MUSHROOMS- 8 TERRE

DONALD W PROTHEROE

PRODUCT ION MANAGER

P O BOX 250

BONNIE TERRE, MO 63628

$314358-3381$

MOREAU S A

HENRI G ROUQUAIROL

OIRECTOR OF APPROVALS

106 BIS AVENUE BEAUREPAIRE

94100 SAINT-MAUR

$0113318861 \times 283$

MORRISON WEIGHING SYSTEHS INC DONALD G MORRISON

PRES

INDIAN 8LUFF RD $80 \times 860$

MILAN, IL 61264

$309799-7311$

MORTEC INOUSTRIES INC

JOE KRAL

515 INDUSTRIAL PK RD POB 977

BRUSH, CO 80723

$303842-5063$

MOS SCALE INTERNATI ONAL

STEVEN E HARRIS

DIRECTOR OF ENGINEERING

3180 REOHILL AVE

COSTA MESA. CA 92626

$714754-7841$

MOTOR VEHICLE MANUF ASSN

HARRY 8 WEAVER 300 NEH CENTER 8 LOG

DETROIT, MI 48202

$313872-4311$

MOULDEN SUPPLY CO

JOHN TI TCOMB

GENERAL MANAGER

3600 HIGHWAY 80 WEST BOX 1247

JACKSON, MS 39205

$601922-4611$

MUTUAL OIL CO INC

EDWARD A RACHINS

PRESIDENT

$P$ O BOX 486

BROCKTON, MA 02403

$617583-5777$
NABISCO BRANDS

SONIA D MEURER

SR LIBRAR I AN

BOX 1942

EAST HANOVER, NJ 07936

$201797-6800 \times 338$

NASH FINCH COMPANY

ROBERT G JOHNSON

3636 STOLLEY PARK ROAO

GRAND ISLANO, NE 68802

$612929-0371$

NATIONAL ASSN OF MARGARINE MFR SIERT F RIEPMA

PRESIDENT

1625 EYE STREET NW SUITE $1024 \mathrm{~A}$

WASHINGTON, DC 20006

$202785-3232$

NATIONAL BARK PRODUCERS ASSN

ROBERT LA GASSE

EXECUTIVE OIRECTOR

13542 UNION YILLAGE CIRCLE

CLIFTON, VA 22024

$703830-5367$

NATIONAL BUREAU OF STANDAROS

CHOU CHI-HSI ANG

DIRECTOR GENERAL

4 TH FL $102 \mathrm{KHANG}-F U$ S ROAD

TAIPEI TAIWAN

NATIONAL BUREAU OF STANDARDS TERRY L GRIMES

SECRETARY

WASHINGTON, DC 20899

NATIONAL BUREAU OF STANDARDS

OAWN M HOFFMAN

SECRETARY

WASHINGTON, DC 20899

NAT IONAL BUREAU OF STANDARDS

CAROLYN K MCKENZIE

SECRETARY

WASHINGTON, DC 20899

NAT IONAL CALIBRATION INC

MARLAN D STAHL

MANAGER

3737 E BROADWAY

PHOENIX, AZ 85040

$602268-1381$

NATIONAL CHEESE INST

ROBERT F ANOERSON

EXECUTIVE DIRECTOR

699 PRINCE ST/PO BOX 20047

ALEXANDRIA, VA 22320

312 263-2733

NAT IONAL CONTROLS INC

BOB BRONSON

MANAGER UF $R \& D$

4500 JOHN YOUNG PARKWAY

ORLANDO, FL 32804

$305298-0506 \times 230$

NATIONAL CONTROLS INC

JAMES C FLINT

PO BOX 15012320 AIRPORT BL VD SANTA ROSA, CA 9540 ?

$707527-5555 \times 284$

NATIONAL CONTROLS INC

PAUL GUNDERSON

INDUSTRIAL PRODUCTS MANAGER

2320 AIRPORTT BLVO PO BOX 1501

SANTA ROSA. CA 95402 
NATIONAL CONTROLS INC GENE I KATZ

VICE PRESIDENT

PO BOX 15012320 AIRPORT BLVD

SANTA ROSA. CA 95402

$707527-5555 \times 303$

NATIONAL CONTROLS INC

DAVID $B$ SHEPARD

VICE PRESIDENT MANUFACTURING

2320 AIRPORT BLVD PO BOX 1501

SANTA ROSA. CA 95402

$707527-5555 \times 131$

NATIONAL FOOD PROCESSORS ASSOC

ALLEN W MATTHYS

DIRECTOR REGULATORY AFFAIRS

1401 NEW YORK AVE NH

WASHING TON. DC 20005

$202331-5960$

NATIONAL GRAIN \& FEED ASSN

MARK G GOEDDE

DIRECTOR OF ENGINEERING SVCS

725 I5TH ST NH PO BOX 28328

WASHINGTON, DC 20005

202 783-2024

NATIONAL LP-GAS ASSN

WILLIAM H BUTTERBAUGH

1301 WEST $22 \mathrm{ND} S$

OAK BROOK, IL 60521

$312986-4800$

NATIONAL OATS COMPANY INC

JERRY GRZESKOWIAK

MANAGER OUALITY CONTROL

$1515 \mathrm{H}$ AVENUE N E

CEDAR RAPIDS: IA 52402

$319364-9161$

NATIONAL PAINT \& COATINGS ASSN

BRUCE H HAMILL

GENERAL COUNSEL

1500 RHODE ISLAND AVE NW

WASHING TON, DC 20005

$202462-6272$

NATIONAL SOFT DRINK ASSOCIATIO

CLAUDIA J LOUIS

MANAGER REGULATORY AFFAIRS

$110116 \mathrm{TH}$ STREET NW

WA SHING TON DC 20036

NATIONAL: STANDARDS COMM.

THOMAS JOSEPH PETRY

EXECUT I VE DIRECTOR

PO BOX 282

NORTH RYDE.

NATIONAL STANDARDS COUNCIL

EXECUTIVE DIRECTOR

$P$ O BOX 5286 BOROKO

PAPUA NEW

NATIONAL STANDARDS OF PR

ANABEL TORRES

GENERAL MANAGER

P O $8 O X$ AC CAPARRA STATION

SAN JUAN, PR 00922

$809751-7188$

NATL METROLOGICAL COORDIN UNIT

JAMES $K$ HUMBLE

DIRECTOR

PO BOX 6 FELL ROAD

CROYDON

$$
01688 \times 1996
$$

NATL READY MIXED CONCRETE ASSN

RICHARD D GAYNOR

VP OF ENGR AND RESEARCH

900 SPRING ST

SILVER SPR ING. MD 20910

$301587-1400$

NATL SEMICOND-DATACHECKER/DTS BOGDAN M MATOGA

ENGINEER ING MANAGER REG COMPL PO BOX 58112

SANTA CLARA, CA 950528112

$408982-3369$

NCR

HENRY L POLITI

PETROL MGMT SYSTMS-USG3

1700 S PATTERSON BLVO

DAYTON, OH 45479

$314991-0273$

NCR CORPORATION

EDHARO BRATLE

RETAIL SYSTEMS D \& P OIVISION WORLO HEADOUARTERS $4 T H$ FLOOR DAYTON. OH 45479

NCR CORPORATION

A R DANIELS

DIRECTOR INDUSTRY STOS $\varepsilon$ REL

1700 S PATTERSON BLVD WHO

DAYTON, OH 45479

$513445-1310$

NCR CORPORATION

JOHN F PAUGSTAT

ENG INEFR ING \& MFG CAMBR IDGE

PO BOX $728 / 800$ COCHRAN AVE

CAMBRIDGE OH 437250523

$614439-0571$

NEBRASKA GRAIN \& FEED DEALERS RICHARD D SANNE

ACT ING D IRECTOR

$1620 \mathrm{M}$ STREET FIRST FLR SOUTH LINCOLN. NE 685082531

$402476-6174$

NEDCO

GAYLORD SMITH

QUALITY CONTROL ASSURANCE DIR 44 UNION ST

FULTONVILLE, NY 12072

NEPTUNE MEASUREMENT CO

DARYL C SHIPMAN

SALES ENGINEER

EMERALO ROAD P $080 \times 792$

GREENWOOD, SC 29646

$803223-1212$

NEPTUNE MEASUREMENT COMPANY

SAM D LOFT IS

VICE PRES SALES \& MARKETING

$P 080 X 792$

GREENWOIOD, SC 29648

NESTLE FOODS CORP

DAVID B HARTLEY SR

ASST MGR OUALITY ASSURANCE

100 BLOOMINGDALE ROAD

WHITE PLAINS, NY 10605

$914682-6857$

NEW BEDFORD SCALE COMPANY

WILL IAM M WHITE

OWNER

507 SMITH NECK ROAD

SOUTH DARTMOUTH, MA 02748

$617997-6730$ 
NEW BRUNSWICK INTERNATL INC JOHN S BAUMANN

VICE PR ESIDENT

5 GREEK LANE

EDISON: NJ 08817

NEW BRUNSHICK INTERNATL INC LOUIS P BONAPACE PRES IDENT 5 GREEK LANE EDISON: NJ 08817 $201287-2288$

NEW BRUNSWICK INTERNATL INC PATRICK MARINO TECHNICAL SER VICE MANAGER 5 GREEK LANE EDISON, NJ 08817 $201287-2288$

NEW HAMPSHIRE RETAIL GROC ASSN JOHN $M$ DUMAIS EXECUTIVE DIRECTOR P 0 BOX 868 MANCHESTER, NH 03105 $603669-9333$

NEW YORK OIL HEATING ASSN

JA HES R WELLS

EXEC VICE PRES 225 SUITE 1202 NEW YORK NY 10122 212 695-1380

\section{NEHPORT ELECTRONICS \\ KARL HINRICHS \\ VP}

630 E YQUNG ST

SANTA ANA: CA 92705

NICOL SCALES INC

NICKI NICOL

PRESIDENT

PO $80 X 222288$

DALLAS, TX 75222

$214428-8181$

NICOL SCALES INC

WILLIAM F NICOL

CHAIRMAN OF BOARD AND CEO

PO BOX 222288

OALLAS, TX 75222

$214428-8181$

NMPC-NMP NUC STAT UNII 2

JOHN M THOMPSON JR

GENERATION ENG HEALTH PHYSICS 8223 PERRUGIA LANE

CLAY. NY 13041

315 425-2525

NORFOLK \& HESTERN RAILHAY CO

LAWRENCE L LOWERY

GENERAL SCALE INSPECTOR

$8 N$ JEFFER SON ST

ROANOKE V VA 24042

$703981-3022 \times 4251$

NORTH 11 TH PO BOX 1037

HARIVIG H SKAUGE

AMERICAN CRYSTAL SUGAR CO

MOORHEAD, MN 56560

$218233-1561$

NORTHEAST ICE CREAM ASSN

AUSTIN T RHOADS

$P$ BOX 0510

FUL TON, MO 20759

$301953-9117$
NORTHEAST PETROLEUM CORP

MALCOLA T NEHELL

VICE PRESIDENT OPERATIONS

295 EASTERN AVE

CHELSEA, MA O2150

$617884-7570$

NORTHEASTERN-PETROLEUM SERESUP JERDHE F MELLO

PRE SIDENT

37 BROOKLEY RO

BOSTON, MA 02130

$617522-8390$

NOR THWEST CALIBRATION SYSTEMS KEITH W CABLE

SLO S LUCILE ST

NORTHWEST SVC STATION EO CO

JOHN H ANOERSEN

PRE SIDENT

2520 NICOLLET AVE

MINNEAPOLIS, MN 55404

$612827-5501$

NORTON CO

ANT HONY $M$ FESTA

GROUP LEADER

1 NEW BOND ST

WORCESTER, MA 01606

$617853-1000 \times 2391$

NORWICH EATON PHARMACEUTICALS LEROY ADAMS

P 0 BOX 2468

GREENVILLE SC 29602

$803277-7110 \times 298$

NOXELL CORP

JOSEPH P ROMANO

HTS \& MEAS INSPECTOR III

IIO5O YORK RD

HUNT VALLEY. MO 21030

$301628-4435$

NY-NJ MILK MARKETING AREA

THOMAS A WILSON

MARKET AOMINISTRATOR

708 THIRD AVENUE

NEW YORK, NY 10017

$212309-1600$

- M SCOTT E SONS COMPANY

VINCENT SNYDER JR

MAR YVILLE. OH 43041

$513644-0011 \times 2497$

OHAUS SCALE CO

MICHAEL P CAMPBELL

PRODUCT MANAGER

29 HANOVER ROAD

FLORHAM PARK. NJ 07932

201 377-9000

OHAUS SCALE CORPORATION

EARL D MYERS II

SENIOR ENGINEER

29 HANOVER ROAD

FLORHAM PARK. NJ 07932

$201377-9000 \times 283$

OHIO COUNTING SCALE NORTH INC

JOHN D GUNN JR

VICE PRESIDENT

4541 INDUSTRIAL PARKWAY

CLEVELAND, OH 44135

$216941-4500$ 
OIL EQUIPMENT SALES \& SVC CO ARTHUR HUBBARD

P D BOX 5096

CHESAPEAKE VA 23324
$804543-3596$

OLIN CORP

JOHN T JOHNS

SCALE REPAIRMAN

SHAMROCK BLDG 246

EAST AL ION: IL 62024

OLSSON \& FRANK

PHILIP C OLSSON

PARTNER

1029 VERMONT AVE NW SUITE 400
WASHINGTON, DC 20005

OMEGA BALANCE SERVICE INC

HER MAN BLITZSTEIN

PRESIDENT

P O BOX 325

MATAWAN, NJ 07747

$201946-2121$

OMYA INC

NORMAN G CHAMPINE

LABORATORY MANAGER

61 MAIN STREET

PROCTOR, VT 05765

$802459-3311 \times 219$

ORMOND INC

ALFRED N ORMOND

PRE SIDENT

12020 EAST RIVERA ROAD

SANTE FE, CA 90670

$213945-1427$

OSCAR MAYER FOODS CORP

CHARLES F COOK

OSCAR MAYER \& CO

P BOX 7188

MAD ISON. HI 53707

$608344-6819$

OSCAR MAYER FOODS CORPORATION DONALD $L$ PAUL

VP OUAL ITY AND REGULATORY AFFR PO BOX 7188

608 ISAL $24-6803$

OTEX FACTOR

JEOFFRE H CHAN

ASSISTANT MANAGER

2040 TAFI AVE

PASAY CITY-MANILA

OWENS CORNING FIBERGLAS

TIMOTHY L SCRIBNER II

PROJECT ENG INEER

300 SUN SHINE ROAD

KANSAS CITY, KS 66102

$913281-9528$

OWENS ILLINOIS INC

D P CONYERS

OUALITY E SPECIFICATIONS MGR 1 SEA GATE TOLEDO OH 43666

$419247-0489$

OWENS-ILLINOIS INC GCD PLT \#9

STEVE C TROUT

$901 N$ SHABBONA ST

STREATOR, IL 61364
OXYGENATED FUELS ASSN

GEORGE S DOHINGUEZ

1330 CONNECTICUT AVE 300

HASHINGTON. DC 200361702

$202659-0060$

PES-AMS US DEPT OF AGRICULTURE JAMES L ALCORN

SUPERVISORY LIVESTOCK SEH SPEC

LIVESTOCK EXCH BLDG ROOM 208

DENVER. CO 80216

$303837-3312$

PA ASSN WEIGHTS AND MEASURES

PEGGY H ADAMS

SECRETAR Y

$P$ O BOX 340

BEDMINSTER, PA 18910

PA POWER \& LIGHT CO

MARSHALL D REHM

FOSSIL FUELS ENGINEER

$2 \mathrm{~N} 9 \mathrm{TH}$ ST

$A L L E N T O W N$
$215821-4714$

PACIFIC SCALE CO

HARRY G BAUGHN

PRES

3236 SE 50TH

PORTLAND, OR 97206

$503777-3978$

PACKAGING CORP OF AHER ICA

GARY BRENNGLASS

PRE SIDEN T

4633 DOWNEY ROAD

LOS ANGELES. CA 90058

$213589-8181$

PAPER PROD DIV PROCTER $\varepsilon$ GAMBL RDBERT STOKES

MGR IND GVT \& REG ACTIVITIES

PO $80 \times 599$

CINCINNATI, OH 45201

$513562-2177$

PAPERBOARD PACKAGING COUNCIL SPENCER A JOHNSON

VICE PRESIDENT

1101 VERMONT AVE NW SUITE 411

WASHINGTON, DC 20005

$202289-4100$

PAR - TEE COMPANY

LAWRENCE J LAUER

GENERAL MANAGER

STATE HIWAY I

SPENCERVILLE. IN 46788

$219238-4483$

PATHMARK SUPERMARKETS

BET TY F GREITZER

MANAGFR-PUBLIC AFFAIRS

301 BLAIR RD

WOODBRIDGE. NJ 07095

$201499-3424$

PAUL MUELLER COMPANY

HARRY BARBEE

SERVICE MANAGER MILK COOLERS

P O BOX 828

SPR INGF I ELD, MO 6580 I

$417831-3000 \times 205$

PAUL SAMSING CONSULTANT

PAUL SAMSING

CONSULTANT

245 NORTH 16 ST

MONTEBFLLO, CA 90640

$213944-6111 \times 323$ 
PEABODY COAL CO ARI ZONA DIV RAYMOND H HELMICK

MANAGER - WE IGHING SY STEMS

1638 E CINNABAR AVE

PHOENIX, AZ 85020

$602943-3837$

\section{PEABODY COAL COMPANY}

STANLEY I CISIEHSKI

MANAGER CUSTOMER SERVICE

$B O X 14495$

ST LOUIS, MO 63178

$618398-7950 \times 222$

PEABODY COAL COMPANY

JOSEPH WHHEATLEY

SCALE TECHNITION

$P$ O BOX 545

GREENVILLE, KY 42345

$502338-1960$

PELOUZE SCALE CO

JOHN H BUNGENER

VICE PRESIDE

EVANSTON, IL 60204

$312328-8330$

PEMBERTON FABRICATORS INC WALTER MERKH

VICE PRESIDENT ENGINEERING

30 INDEL AVE

RANCOCAS, NJ 08073

$609267-0922$

PENN CHAMP INC

PAUL SIMITOSKI

LABORATORY DIRECTOR

$14 T H$ \& RAILROAD ST

EAST BUTLER, PA 16029

$412287-8771 \times 33$

PENNSYL VANIA SCALE CO

ERIC A GARD

VP \& OPERATIONS MGR

12 GRAYBILL AVE

LEOLA, PA 17540
$717656-2653 \times 42$

PENNSYLVANIA SCALE COMPANY BARBARA S UMBENHAUER

TECHNICAL WRITER

21 GRAYBILL RD

LEOLA. PA 17540

$717656-2653 \times 53$

PEPSI COLA CO

GEORGE E GARDNER

3 GANNETT DR 2ND FLOOR

WHITE PLAINS, NY 10604

PERDUE INC

ALAN P CULVER

P O BOX 1537

SALISBURY. MD 21801

PERSONAL PRODUCTS COMPANY

ARTHUR I HUETTEMAN

DIR REG ULATORY AFFAIRS

VAN LIEW AVE

MILLTOWN. NJ 08850

$201524-7294$

PETER ECKRICH AND SONS INC LARRY M RASMUSSEN

QUALITY ASSURANCE ENGINEER $P$ O BOX 388

FORT HAYNE, IN 46801

$219481-2034$
PETERSON PURITAN INC

BEN Z TAYLOR

TECHNICAL DIRECTOR

HEGELER LN

DANVILLE: IL 61832

PETROLEUM CONTRACTORS INC

TIM MCGUIGAN

BRANCH MANAGER

RD 6 BOX $579 F$

ALLENTOWN, PA 18106

$215398-0421$

PETROLEUM EOUIP INSTITUTE

HOWARD UPTON

EXEC VICE PRES

PO BOX 2380

TULSA. OK 74101

$918743-9941$

PETROLEUM EQUIPMENT EMAINTENACE BARRY G FOSDICK

OWNER

PO BOX 53

BASSETT HI 53101

$414877-2661$

PETROLFUM MARKETERS ASSN OF AM BARBARA J FAULKNER

VICE PRESIDENT

1120 VERMONT AVE SUITE 1130

WASHINGTON, DC 20005

$202331-1298$

PFIZER INC

JOHN NATISHAN

DIRECTOR OUALI TY CONTROL

100 JEFFER SON ROAD

PAR SIPPANY. NJ 07054

PHILIP A HUNT CHEMICAL CORP

GLENN C KNUDSEN

PACKAGING MANAGER

5 GARRET MOUNTAIN PLAZA

WEST PATERSON. NJ 07424

$201944-4000$

PHILLIPS PETROLEUM COMPANY

C I TAVERNER

PRINC IPAL TECH REPRESENTATIVE

11 D-4 PHILLIPS BLDG

BAR JLESVILLE. OK 74004

$918 \quad 661-4944$

PILLSBURY CO

CARL A SMITH JR

DIRECTOR PRODUCT SAFETY - AGRI

PILLSBURY CENTER

MINNEAPOL IS. MN 55402

$612330-5165$

PILLSBURY COMPANY

HOWARD E BAUMAN

VP SCIENCE E REGULATORY AFFAIR

311 SECOND ST SE

MINNEAPOLIS. MN 55414

$612330-4676$

PILLSBURY COMPANY

RONALD FREYER

MANAGER QUALITY GROCERY

PILLSBURY CENTER

MINNEAPOLIS. MN 55402

PILLSBURY COMPANY-MS 9441

CARL A SMITH JR

DIRECTOR OA TECHNICAL SERVICES

311 SECOND STREET S E

MINNEAPOLIS. MN 55414

$612330-4477$ 
PI INEY BOWES

RUTHERF ORD H FENN

DIRECTOR CORPORATE STANDARDS

WAL TER WHEELER DR IVE

STAMFOR O. CT 06926

$203853-0727$

PI TNEY BOWES

OON J VIOLETTE

380 MAIN AVE

NORWALK. CT 06852

$203853-7113$

PI TNEY BOWES

MARL C WESTFALL

380 MAIN AVE

NORWALK: CT 06852

$203356-5541$

PITNEY BOWES INC MSD

FREO J STAUDI NGER

BUSINESS SEGMENT MANAGER

WAL TER H WHEELER JR OR IVE

ST AMFORO, CT 06926

203 356-7019 $\times 404$

PITNEY-BOHES INC

GARY G HANSEN

ENG INEER ING MANAGER

380 MAIN AYE

NORWALK CT 06852

$203854-7279$

PITT TRAINING ASSOCIATES

HY PITT

PRESIOENT

$6810 \mathrm{~N}$ NEIL PL

MIL WAUKEE: WI 53209

PITTSBRG-MIOWAY COAL MINING CO

WILLIAM T BOWLES

$D I R E C T O R$ PREP ENG INEER ING

1720 S BELLAIRE ST

OENVER : CO 80222

PLANTERS $\varepsilon$ LIFESAVERS

PHIL SHEET

QUALI TY ASSURANCE TECHNOLOGIST

200 JOHNSON AVENUE

SUFFOLK. VA 23434

$804934-6436$

PLASTICS INC

JOHN O MOYER

QUALITY CONTROL MANAGER

P O BOX 43440

ST PAUL. MN 55164

\section{PMP COR PORATION}

RICHARO L HOCKMUTH

MECHANI CAL PRODUCTS ENGR MGR

25 SECURITY DRIVE POB 422

AVON, CT 06001

$203677-9656$

POLK OIL CO INC

VERNON T HUMPHR IES

MAINTENANCE SUPVT

1422 SOUTH 1 ST

LUFKIN. TX 75901

$409634-8484$

POLY-AMERICA INC

WILLIAM C NEAL

$\checkmark I C E$ PRESIOENT-MARKETING

2000 W MARSHALL

GRANO PRAIRIE. TX 75051

$214647-2950$
POLY-TECH INC

JEFFREY N HERSTEIN

D IRECTOR OF SALES

1401 WEST 94 TH STREET

MINNEAPOLIS. MN 55431

$612884-7281 \times 232$

POPSICLE INDUSTRIES INC

MAX S STURGES

MGR OUALITY ASSURANCE F FLAVOR

110 ROUTE 4 P 0 BOX 200

ENGLEWOOO, NJ 07631

$201567-8500$

PORT OF CORPUS CHRISTI AUTH

JOE GIANNINA

SUPR BULK MATERIALS DOCKS

P O BOX 1541

CORPUS CHRISTI. TX 78403

$512882-1956$

POWELL ALL-STEEL SCALES INC

MAR IAN P BAIR

RT 8 BOX 313

JASPER, AL 35501

PRECISION SCALE

MERRILL E THUR MAN

SCALE MAINTENANCE ENGINEER

$720 \mathrm{~N}$ EL MOOD

SPR INGFIELD. MO 65802
$417831-0995$

PRECISION HEIGHT SCALE CO

DANIEL A KENDRA

315 R ICKERT ROAO

SELLERSVILLE, PA 18960

$215723-9523$

PRESTO PRODUCTS INC

ROBER A LE CAIRE

DIRECTOR-LABORATORY SERVICES $P 0$ BกX 2399

APPLETON. WI 54913

$414739-9471$

PRESTO PROOUCTS INCORPORATED

PAUL ZIEMER

ART DIRECTOR/PACKAGING

$B O X 2399$

APPLETON, WI 54913

$414739-9471$

PRINCE FOODS INC

FAROOK TAUFIO

VICE PRESIDENT OUALITY ASSUR

PRINCE AVENUE

LOWELL. MA 01853

$617458-4111 \times 121$

PROCTER \& GAMBLE

YILLIAM H BRAUN

PACKAGING SECIION HEAD

6100 CENTER HILL RD

CINCINNATI. OH 45224

$513659-5233$

PROCTER 6 GAMBLE CO

GEORGE E CARLETON

MGR ADMIN E SPECIAL SERVICES

ONE PROCTER E GAMBLE PLALA

CINCINNATI: OH 45202

$513562-2721$

PROCTER \& GAMBLE CO

ROBERT S RICHARDS

BAL ANCE ANO SCALE REPAIRMAN

6083 CENTER HILL RD

CINCINNATI. OH 45224

$513659-7073$ 
PROCTER \& GAMBLE COMPANY

VIC TOR L PRE STON

BAL ANCE $E$ SCALE REPAIRMAN

6083 CENTER HILL RD

CINCINNAII 3 OH 45224

$513659-7073$

PROCTER \& GAMBLE COMPANY

WILLIAH A SHITH

II 520 REED HARTHAN HW/PO 41520

$513763-6075$ OH 45241

PROCTER \& GAMBLE COMPANY

HARR I SON B WILSON III

LEGAL DIVISION COUNSEL

ONE PROCTER \& GAMBLE PLZ/8599

CINCINNATI OH 45202

$513983-5873$

PROCTER E GAMBLE MFG CD -

R J KATHMANN

1601 WE ST TTH ST

LONG BEACH, CA 90813

PROCTER ANO GAMBLE

LOUIS K HILLER

REGULATORY RELATIONS - FOODS

6083 CENIER HILL RD

CINCINNATI OH 45224

$513659-7291$

PROCTER AND GAMBLE CO

LEKENNETH HILLER REL FOOD DIV

HEAD RE GULATORY REL

CINCINNATI, OH 45224

$513977-7969$

PRODUCT ASSURANCES

HAR VEY SCHDCK JR

CONSULT TANT

30 OAK RIDGE DR

HADDONFIELD, NJ 08033

$609428-7236$

PUBLIX SUPER MARKETS INC

HENRY E CROSBY

ASST DIR CONSTRUCTION E MAINT

$P$ O BOX 407

LAKELAND FL 33802

$813686-1188 \times 281$

PUMP PARTS INC

RONALD A PATTERSON

PRESIDENT

12427 GRAND RIVER

DETROIT. MI 48204

$313933-9500$

PHI INC ORPORATED

STEPHEN $J$ HIEBER

OWNER

211 LINCOLNWAY H

NEH OXFORD,PA 17350

$717624-2111 \times 25$

O C INC QUAL CONTR LAB DIV

A F 2 IMMERMANN

PO BOX 514

SOUTHAMPTON, PA 18966

QUAKER OATS

ROY ANDERSON

QUALITY AS SURANCE

PO BDX 3040 FOODS DIVISION

SHIREMANSTOWN, PA 17011

$717737-8601 \times 282$
OUAKER OATS COMPANY

JOHN R MCCLURE

OUALITY ASSURANCE MANAGER

PO BOX 1677

CHATTANDOGA. TN 37401

$615698-1591 \times 178$

QUALITY METER INC

ALAN CAMERON

MANAGER QUALITY ASSURANCE

507 MISSOURI AVENUE

HEST PLAINS. MO 65775

$201687-4000$

QUALIIY PUMP \& ELECTRIC INC

JOHN K KOLB

ROCK SPRINGS, WY 82901

$R$ H ALEXANDER CO

KENNETH WTEEN

PRESI DENT

3001 E I2TH ST

LOS ANGELES, CA 90023

$213 \quad 264-1141$

$R$ MCCOLLUM CO INC

RICHARD R SAUNDER S

SEC \& TREAS

107 MEADOWVIEW RO BOX 20145

GREENSBORO, NC 27420

$919272-6191$

RALSTON PURINA CO

EDWARD L BERGMANN

MGR QUALITY ASSURANCE PET FOOD

900 CHECKERBOARD SOUARE

ST LOUIS, MO 63188

$314982-3483$

RAMSEY ENGINEER ING COMPANY

MAX C CASANOVA

MANAGER FIELD SERVICE

1853 H COUNTY RD C

SAINT PAUL MN 55113

$612633-5150 \times 264$

RAMSEY ENGINEER ING COMPANY

PAUL CHASE

VICE PRESIDENT OF RES \& DEVEL

1853 W COUNTRY ROAD C

ST PAUL, MN 55113

$612633-5150$

RASCHER \& BETZOLO INC

CHARLES RASCHER JR

5410 N DAMEN AVE

$312275-7300$

RAY EOUIPMENT CO INC

ROGER H FORD

OFF ICE MANAGER

5538 MERRIAM DR

MERRI AM, KS 66203

$913384-1070$

RE-MI FOOD PRODUCTS

LEE BROWN

CONTINENTAL FOOD PRODUCTS CO

1201 TONNE ROAD

$\begin{array}{ll}\text { ELK GROVE: IL } 60007 \\ 312 & 640-0400\end{array}$

RED-ROSE TRANSFER INC

JAY-DONALD FISHER

PRE SIDENT

1526 IILTMORE AVE

LANCASTER. PA 17601

717 397-0953 
REED TRUCKING

GORDON F REED

PRESIDENT

60 MELR OSE ROAD

GRAND I SLAND, NY 14072

REFINED SUGARS INC

JAMES REICHE

TECHNICAL PROJECTS MANAGER

1 FEDERAL STREET

YONKERS. NY 10702

$914963-2400$

REGULTECH ASSOCIATES INC

PAUL KHAN

PRESIDENT

$158 \mathrm{~W}$ BOSTON POST RD/POB 717

MAMARONECK, NY 10543

$914899-0224$

RENEHABLE FUELS ASSOC

ERIC VAUGHN

PRESIDENT/CEO 499 SO CAPITOL ST SW SUITE 420

499 SO CAPITOL ST SW
HASHING TON. DC 20003

$202484-9320$

RETAIL DATA SYSTEMS

ROBERT MURPHY

SERVICE MANAGER

106 GREYSTONE AVE

KANSAS CITY KS 66103

$913281-1333$

REVERE CORP OF AMER ICA

CARL R KUTELIS

DIRECTOR OF ENGINEER ING

P O BOX 56

WALLINGFORD, CT 06492

$203269-7701$

REVERE CORPORATION OF AMERICA

JOHN J ELENGO JR

VICE PRESIDENT

$845 \mathrm{~N}$ COLONY RD P $\mathrm{O}$ BOX 56

HALLINGFORD, CT 06492

$203269-7701$

REX PLASTICS INCORPORATED

RICHARD C PHELPS

DIRECTOR OF MANUFACTUR ING

1200 UNITY

THOMASVILLE, NC 27360

REXWORKS INC-CONST MACH DIV MAIT JACOBCHICK

MANAGER ENGINEER ING \& SERVICE

$445 \mathrm{~W}$ OKLAHOMA AVE BLDG F2

MILWAUKEE, WI 53207

$414643-2230$

REYNOLDS METALS CO

GERALD E MER ICLE

MANAGER $-R \& R$ TECHNOLOGY

1803 RE YME T ROAD

RICHMOND VA 23237
$804743-6757$

REYNOLDS METALS COMPANY

ER VIN G RICHARDSON

CORPORATE ENG GOVT STANDARDS

$6601 W$ BROAD ST

RICHMOND, VA 23261

$804281-2276$

REYNOLDS METALS COMPANY

J R WAL LACE

SHEFFIELD, AL 35660

$205386-6000$
RICE LAKE BEAR ING INC

RICHARD CALKINS

MET ROLOG IST

RICE LAKE, WI 54868

715 234-9171

RIEDE SYSTEMS INC

JOHN W SCHULT2

SALES MANAGER

8375 SUNSET RD

MINNEAPOLIS, MN 55432

$612784-8020$

RIGHT HEIGH SCALE CO

DAVID M HEBENSTREIT

PRESIDENT

RT 10 HWY $80 E$ AIRPORT IND PK

PEARL, MS 39208

$601932-4790$

RIV IANA FOODS

TED R LABREE

TECHNICAL MANAGER REG SERVICES

1 TO2 TAYLOR STREET

HOUSTON. IX 77007

$713529-3251 \times 854$

\section{ROBERT H KELLEN COMPANY}

ROBERT H KELLEN

PRESIDENT

5775 D PEACHTREE DUNWOODY RD

ATL ANTA. GA 30342

$404252-3663$

ROSS IND DEPT OF CARGILL INC

ROBERT W SCHRADER

MGR LAB \& TECH SERV

$P 080 \times 2696$

HICHITA. KS 67201

$316267-6281 \times 250$

ROSS LABORATOR IES

MICHAEL H HANEY

DIR REGULATORY AFFAIRS

625 CLEVELAND AVE

COLUMBUS. OH 43216

$614227-3212$

$S$ C JOHNSON \& SON INC

RICHARD $M$ NEU

0 A TECHNOLOGIST

1525 HOWE ST MS 273

RAC INE. II 534

$414631-3713$

$S W B E I Z C O I N C$

FREDERICK $W$ BETZ

2239 KIRK AVE

BAL IIMORE, MD 21218

$301366-1030$

SAFEWAY STORES INC

C A STOFFERS

MGR FOOD TECHNOLOGY DIVN

430-A JACK SON ST

OAKLAND. CA 94660

$415891-3250$

SALTER INDUSTRIAL MEASUREMENI A G COOPER

MANAGING DIRECTOR

GEORGE STREET

WFS T BROMWICH

SAMUEI K SPIGLER COMPANY INC

SAMUEL K SPIGLER SR

PRE SI DENT

$P \cap B \cap X 360$

FUNKSTOWN. MD 21734

$301739-4462$ 
SANDERS ASSOCIATES INC HARRY 8 HAYMES

95 CANAL ST

NASHUA. NH 03061

$603885-4913$

SANDIA NATIONAL LABORATORIES

OL I VER D ROHRBACK

DIVISION 8416

IIVERMORE CA 94550

$415 \quad 422-3146$

SANITARY SCALE COMPANY

THOMAS L NOLAN JR

PRESIDENT

910 E INCOLN AVE

BELVIOERE, IL 61008

815 544-2i81

SAR TORI US BALANCES/BRI NKMANN DIETER F SCHLUTER

$\checkmark p$

CANTIAGUE RD

WESTBURY NY 11590
$516334-7500 \times 230^{\circ}$

SCALE MANUFACTURERS ASSN

RAYMOND $J$ LLOYD

EXECUTIVE DIRECTOR

152 ROLL INS AVE SUITE 208 ROCKVILLE, MD 20852

$301984-9080$

SCALE MANUFACTURERS ASSN

ROBERT A REINFRIED

TECHNICAL ASSISTANT

152 ROLLINS AVE

ROCKVILLE, MD 20852

$301984-9080$

SCALE MANUFACTURERS ASSN

DARYL E TONINI

TECHNICAL DIRECTOR

6842 ELM STREET SUITE 102

MCLEAN, VA 22101

$301-984-9080$

SCALE PEOPLE INC

RONALD L BUCK

10101 BACON DR

BELTSVILLE, MD 20705

$301949-6994 \times 215$

SCALE SERVICE CO

JAMES D SUGGS

$4406 \mathrm{~N}$ OAKLEY

KANSAS CITY, MO 64117

$816454-9509$

SCALE SYSTEMS HAWAI I

GLENN C SHIROMA

GENERAL PARTNER

$P$ O BOX 1598

HILO, HI 96720

$808935-1344$

SCHERING CORPORATION

ROBERT BIXLER

1011 MORRIS ST

UNION. NJ 07083

$201931-2000$

SCHNEIDER SYSTEMS

MILES A SCHNEIDER

PRESIDENT AND GENERAL MANAGER 621 HEA VER ST

FAIRFIELD, OH 45014

$513896-7900$
SCHRE IAER FOODS INCORPORATED DONALD E CRIPE OUALITY SYSTEMS CORPORATE DIR P O BOX 19010 O 54307

$414437-7601 \times 581$

SCHWANS SALES ENTERPRISES

PHIL RYBACKI

NATIONAL RETAIL MKTG MGR

MAR SHALL, MN 56258

$507532-3274$

SCIENCE APPLICATIONS INTL CORP STANLEY CRANDON

SCIENCE APPLICATIONS INC

POMONA. CA 91766

$714623-6711$

SEABOARD SYSIEM RAILROAD FUPOE LIOYD JR P O BOX 45052

JACKSONVILLE. FL 322325052

$904359-1024$

SEEDBURO EQUIP CO

JACK T SNADER

EXECUTIVE VICE PRESIDENT

1022 JACKSON BLVD

CHICAGO IL 60607

$312738-3700$

SELF-EMPLOYED

RALPH T DICKSON

SCALE TECHNICI AN

RT 3 BOX 246-E

CHINA GROVE. NC 28023

$704938-2085$

SEL IEC CORPORATION

JAMES M NOBLE

PRES I DENT

4568 ENTERPRISE ST

FREMONT. CA 94538

415 657-7800

SENSITIVE MEASUREMENT INC

RAYMOND R WELLS

$\checkmark I C E$ PRES - SALES

P O BOX 72

PFMBERTON. NJ 08068

$609894-2292$

SERAPHIN TEST MEASURE

CHARLES H GARDNER

PRESI DENT

10 INDEL AVE

RANCOCAS, NJ 08073

$609267-0922$

SER VICE EQUIPMENT COMPANY INC RON THOMLEY

ASSI STANT MANAGER

502 EAST MAIN STREET

DOTHAN, AL 36301

$205793-6676$

SERVICE STATION DEALERS OF AME VIC RASHEED

EXECUTIVE DIRECTOR

400 NORIH CAPITOL ST SUITE 175 WASHINGTON, DC 20006

202 393-6868

SGS CONTROL SERVICES INC

NORMAN A GREEN

VICE PRESIDENI

42 BROADWAY

NEW YORK. NY 10004

$212482-8700 \times 226$ 
SHAMROCK SCALE COMPANY JAMES W JONES

5553 JEFFREY LANEIPO BOX 1719 MORRISTOHN. TN 37814

$615586-2083$

SHAMROCK SCALE COMPANY

CONOR O DONOGHUE

PRESIDENT

5553 JEFFREY LANE/PO BDX 1719

MORRIST OWN, TN 37814

$615586-2083$

SHASTA BEVERAGES INC

JOSEPH B LEWIS

MGR QUALITY EASTERN STATES

6085 BARFIELD ROAD SUITE 204

ATLANTA. GA 30328

SHELL OIL CO

$G$ RICHARD FURBER

STAFF DISTR IBUTION REPR

THO SHELL PLAZA-RM 1508

HOUSTON. TX 77001

$713241-4950$

SHELL OIL COMPANY

N E FRA ILEY

PACKAGING COORD LUBRICANTS

$P$ O BOX 2463

HOUSTON . TX 77001

$713241-2689$

SHELL OIL COMPANY

CHARLES MICHELL JR

$S R$ LOSS CONTROL REPRESENTATIVE TSP ROOM 1140

HOUSTON, TX 77001

$713241-0455$

SHELL OIL COMPANY

CHARLES L VAN INWAGEN

SENIOR STAFF ENGINEER

1200 MI LAM PO BOX 3105

HOUSTON. TK 772533105

$713241-1778$

SHERWIN-HILLIAMS COMPANY

DWIGHT A CDHAGAN

DIR REGULATORY SERVICES

101 PROSPECT AVE NW

CLEVELAND: OH 44115

$216566-2918$

SI HCO

CARL L QUINN

PRESIDENT

382 MARTIN AVE

SANTA CLARA CA 95050

$408727-3611$

SINGLE SERVICE INSTITUTE

JOSEPH H BOW

1025 CONNECTICUT AVE NW

WASHINGTON, DC 20036

$202347-0020$

SIOUXLAND SCALE SERYICE INC

HILLIAM R HANNA

$1218 \mathrm{~N}$ LEHIS BLVO

SIDUX CITY. IA 51105

SMITH METER INC

PHILIP E SHANSON

SR ENG I NEER

1602 WAGNER AVE

ERIE, PA 16514

814899-0661 X268
SMITHS SCALE SERVICE INC JAMES L SMITH

PRES IDENT

$25098 \mathrm{TH}$ AVE

COUNCIL BLUFFS. IA 51501

$712322-8671$

SNAP-ON TOOLS CORP

RICHARD M SHIRVEN

SERVICE MANAGER

280180 TH ST

KENOSHA. WI 53140

$414656-5391$

SOAP \& DETERGENT ASSN

MARY P KILCOYNE

DIRECTOR LEG \& REG INFO

475 PARK AVE $S$

NEW YORK. NY 10016

$212725-1262 \times 21$

SOUTH SHORE SCALE CO

HAMILTON T TEWKSBURY

81 ELM ST
COHASSETT. MA 02025

SOUTHERN CO SERVICES FUEL DEPT JACK C AWBREY

QUALITY CONTROL SPECIALIST

1036 2ND STREET N E

ALABASTER, AL 35007

$205877-7675$

SOUTHERN COMPANY SERVICES

WILLIAM D BRASHER

OUALITY CONTROL SPECIALIST

$P$ D BDX 2625

BIRMINGHAM. AL 35202

$205877-7653$

SOUTHERN PACIFIC TRANSP CO

EDW IN G BANKS

SUPERVISOR OF SCALES

1 MARKET PLAZA ROOM 1007

SAN FRANC ISCO. CA 94105

$415891-7828$

SOUTHERN PUMP \& TANK CO

LARRY F HUNTER

MANAGER TECHNICAL SERVICES

P 0 BDX 31516

CHARLOTTE. NC 28231

$704596-4373 \times 230$

SOUTHERN RAILWAY SYSTEM

GROVER L SCHENCK III

SUPT SCALES E HIGHWAY EOUIP

99 SPRING ST SH

A TLANTA: GA 30303

404 529-1391

SOUTHERN TEA COMPANY

GLENDA ROGERS

OUAL IIY AS SURANCE MANAGER

1267 COBB INDUSTR IAL DR IVE

MARIETTA. GA 30066

$404428-5555$

SOUTHERN WEIGHING \& INSP AU

MARTIN $R$ GRUBER JR

DIRECTOR OF SCALE TESTING

151 EL.LIS ST NE 306

A TL ANTA. GA 30303

$404659-6266 \times 266$

SOUTHLAND CORPORATION

CHARLES R PALMOUIST

GASOL INE SUPERVISOR

8825 ROE AVE

PRAIRIE VILLAGE, KS 662072299

$913649-8220$ 
SOUTHLAND CORPORATION

ROBERTO PERAZA

GASOL INE CONSTRUCTION MANAGER

$P$ O BOX 719

DALLAS: TX 75221

$214559-0 B 50$

SOUTHWEST PUMP COMP ANY

F MLCHAEL BELUE

MECHANICAL ENGINEER ING DEPT

PO DRAHER 280

BONHAM, TX 75418

$214583-3134 \times 47$

SOUTHHESTERN PUBLIC SERVICE CO DHIGHT G ROBERT SON

SEN IOR ENG INEER-P ER FOR MANCE

GTH $E$ TYLER

AMAR ILLO TX 79170

$806378-2722$

SPECIALTY FOOD EQUI PMENT CO

RICHARD E OGREN

PRESIDENT

306 DELAHARE

KANSAS CITY, MO 64105

$816842-1773$

SPEEDWAY PETROLEUM

RON G SCHUMANN

MANAGER MAINTENANCE

2121 DIRECTORS ROW

IND I ANAPOL IS. IN 46241

$317243-7500^{\circ} \times 224$

SPIKES FOOD \& MEAT EQUIPAENT ORVILLE E SPILKER

PRESIDENT

7629 ENGLEWOOD

RAYTOHN MO 64138

$816358-4600$

SPRAYON PRODUCTS

ROBERT A GRAHAM

TECHNICAL DIRECTOR

26300 FARGO AVE

BEDFORD HEIGHTS, OH 44146

$216292-7400 \times 270$

STABRO LABORATORIES INC

DAVID N EBBERT

MANAGER

25 KENSINGTON AVE

SALTLAKE, UT 84115

$801487-1344$

STALEY CONTINENTAL INC

$N$ RICHARD LOCKMILLER

MANAGER GDVERNMENT RELATIONS

$P$ O BOX 2513

DECATUR, IL 62525

$217421-3065$

STANDARD OIL COMPANY

LEE HOCK

FIELD SERVICES CODRDINATOR

4850 EAST 49 TH STREET

CLEVELAND, OH 44125

$216271-8211$

SIANDARD OIL COMPANY (OHIO)

JAMES J QUANDT

SENIDR AUTOMOTIVE SPECIALIST

4850 E 49 TH ST

CLEVELAND, OH 44125

$216575-4383$

STANLEY TOOLS

PARKER A THOMPSON

MARKETING MANAGER

600 MYR TLE ST

NEW BRITAIN. CT 06050
STANLEY TOOLS-DV STANLEY WORKS

DONALD P RYAN

CHIEF DR AFTSHAN

600 MYRTLE ST

NEW BRITAIN, CT 06050

$203225-5111 \times 453$

STATE OF ILLINOIS

RALPH H MILLER JR

COMMISSIONER INDUSTR IAL COMMN

100 W R ANDOLPH ST STE $241-C$

CHICAGO: IL 60601

STAUFFER CHEMICAL COMPANY

TERRELL L MAYS

DIRECTOR-P ACKAG ING

WESTPORT, CT 06881

$203222-3390$

STE INHOFFER SCALE CO INC

GLEN E BABCOCK

SALES MANAGER

55645 CURRANT ROAD

MISHAWAKA. IN 46545

$219259-5425$

STERLING SCALE CO INC

ERIC D DIXON

PRES \& GEN MGR

20950 BDENING DRIVE

SOUTHFIELD, MI 48075

$313358-0590 \times 25$

STRAINSERT COMPANY

ALEXANDER YORGIADIS

VICE PRESIDENT

1404 GARZA ST

ANAHEIM, CA 92804

$714827-3803$

STROH BREWING CO

DONALD P RICE

ASST DIR QUALITY ASSURANCE

100 RIVER PLACE

DETROIJ: MI 48207

$313446-2157$

SUBURBAN PROPANE GAS CORP

JOHN R KUKUCKA

VICE PRESIDENT ENGINEER ING

RT 10 PO BOX 206

HHIPPANY, NJ 07981

$201887-5300 \times 366$

SUBURBAN PROPANE GAS CORP

MELVIN W SCHROEDER

MANAGER REGULATORY 6 SAF SERV

P D BOX 206

HHI PPANY NJ 08807

$201887-5300$

SUN REFINING \& MARKETING

TERRY ROSFELDER

1801 MARKET ST

PHILADELPHIA. PA 19103

$215977-6502$

SUN-DIAMOND GRDWERS OF CALIF

$R$ E GUNNERSON

DIRECTOR OUALI IY ASSURANCE

1050 S DI AMOND ST PO BOX 1727

STOCKTON CA 95201

$209466-4851$

SUNNYSIDE CORPORATION

WILLI AM W BUCHANAN

REGULATORY AFFAIRS

225 CARPENTER AVENUE

WHEELING IL 60090
$312541-5700$
$\times 0022$ 
SUPERIOR EQUIPMENT COMPANY INC JAMES H SPIROS

3283 IVANHOE AVE

SAINT LDUIS: MO 63139

SHEDA INTERNATIONAL INC

BARBARA J MUELLER

MANAGER US OPERATIONS

34 MAPLE AVE

PINE BROOK. NJ 07058

$201575-8100 \times 303$

SHEDA INTERNATIONAL INC

DANIEL ROMAN

QUALITY CONTROL ENGINEER

34 MAPLE AVE P

201 575-8100

SYSTEMS ASSOCIATES

INC ORPORATE

ROBERT T

205 PETERSON RD

IIBERTYVILLE. IL 60048

SYSTEMS INSTALL SPECS CO INC

HUGO M CANCIO

ENG INEER ING MANAGER

PO BOX 10310 CAPARRA HGHTS STA SAN JUAN, PR 00922

809 783-1057

SYSTEMS SERVICES

PRESIDENT

$P$ BOX 65

BREMEN. OH 43107

$614878-4269$

TANNER OIL CO

ROBERT B TANNER

SECRE TARY

620 S FRONT ST

IRONTON OH 45638

$614532-9145$

TEC AMERICA INC

DENNIS SCHAF FER

SCALE SERVICE COORD INATOR

19250 VAN NESS AVE

TORRANCE, CA 90501

$213320-8900$

TECHSCIENCE INTERNATIONAL INC RICHARD D DUNCAN

SER VICE MGR

3020 NO HESPERIAN WAY

SANTA ANA, CA 92706

$714558-6911$

TECNETICS INDUSTRIES

GERALD J BERGER

SALES MANAGER

2180 OLD HIGHWAY 8

ST PAUL. MN 55112

$612780 \div 4880$

TENCO DIVISION OF TETLEY INC ANGELO GIAMMARI NO

OUALITY ASSURANCE DIRECTOR

$P$ O BOX 15

LINDEN, NJ 07036
$201862-6600 \times 283$

TETLEY INC

KENNETH MAIIETTA

QUALITY CONTROL

P O BOX 3337

WILLIAMSPORT, PA 17701
TETLEY INC

PHILIP J MCGOWAN

MANAGER OF OUALITY ASSURANCE

W RUBY E GRAND AVE

PALISADES PARK, NJ 07650

201 943-0600

TEXAS SCALES AND MATERIAL HDLG JOHN J CHINNI

PRESIDENT GEN MGR

RT 3 BOX $183 \mathrm{D}$

CIBOLO, TX 78108

$817848-4491$

TEXIZE DIV OF DOH CHEMICAL CO LARRY D SOLE

DIRECTOR OUALITY ASSURANCE

PO BOX 368

GREENVILLE: SC 29602

$803963-4261$

THAYER SCALE DIV HYER IND

FRANK $S$ HYER

PRESIDENT

RT 139

PEMBROKE MA 02359

THE OUAKER OATS CO

HAROLD D GODSEY

MGR PROCESS ENG INEER ING

MERCHANDISE MART

CHICAGO, IL 60654

$312222-7600$

THE OUAKER OATS COMPANY

STEVE GRUL ER

MANAGER OUALITY AS SURANCE

400 SECOND STREET N E

CEDAR RAPIDS. IA 52406

$319362-3121 \times 126$

THE OUAKER OATS COMPANY

MINDAUGAS A SIMKUS

MANAGER OF PROCESS ENGINEERING

345 MERCHANDISE MART

CHICAGח, IL 60654

$816279-1651 \times 326$

THE OUAKER OATS COMPANY

TOM TOPAL I S

MGR OUAL ASSURANCE-COMPLIANCE

GIT WEST MAIN ST

BARRINGTON. IL 60010

$312381-1980 \times 2064$

THOMAS J LIPTON INC

DEBORAH J HORNER

OUALITY CONTROL MANAGER

2200 DELAWARE AVE

SANTA CRUZ CA 95060

$408426-9100 \times 0336$

THOMAS J LIPION INC

JOHN C YOUNG

ASS ISTANT/GENERAL COUNSEL

800 SYLVAN AVE

ENGLEWDOD CLIFFS. NJ 07632

THURSTON AVIATION INC.

ROY S BERRYHILL

VEHICLE MAINTENANCE

3840 AIRPORT DRIVE

CHARLOITE, NC 28219

$704394-4331$

TIME OIL CO

JOHN P DENHAM

SYSTEMS ENCINEER

2737 WOMMODORE WAY

SEATTLE. WA 98199

$206>85-2400 \times 61$ 
TOBAL DIV VERTEX INDUSTRIES CLARENCE I KASLINE PRODUCT MARKETING MANAGER 23 CAROL ST CLIFTON: NJ 07014

TOKHEIM CORPORATION

WALTER F GERDOM JR MANAGER-TECHNICAL SERVICES P O BOX 360 FORT WAYNE, IN 46801

$219423-2552 \times 316$

TOKHEIM CORPORATION

HILLIAM D KEY

MANAAGER OF ENGINEER ING

PO BOX 360

FT WAYNE IN 46801

$219493-2554 \times 345$

TOKHEIM CORPORATION

RICHARD TUCKER

ASST MANAGER CUSTOMER SERVICE P D BOX 360

FT WAYNE IN 46801

$219423-2552$

TOKHEIM FCSI

CHRISTOPHER VAN METRE

TECHNICAL SUPPOR

600 CEN TURY PLAZA

HOUSTON, TX 77073

$713821-9865$

TOKYO ELECTRIC USA INC

SEIJI SUZUKI

19250 VAN NESS AVE

TORRENCE CA 90501

$213320-8900$

TOKYO ELECTRIC USA INC YUKIHIRO TAKADO

PRODUCT MANAGER

19250 VAN NESS AVENUE

TORRENCE. CA 90501

$213956-2614$

TOLEDO SCALE

THOMAS M STABLER

MANAGER - WTS AND MEAS

$P$ O BOX 658

HORTHING TON, OH 43085

$614438-4548$

TOTAL PETROLEUM INC

DAVID L STRONG

REGIONAL MANAGER

$1019 \mathrm{~N}$ BURL INGTON

$N$ KANSAS, MO 64116

$816421-2200$

TOTALCOMP INC

RUDOLPH J KOLACI

PRE SIDENT

3-09 SADDLE RIVER ROAD

FAIR LAWN NJ 07410

$201797-2718$

TRANSDUCERS INC

LAVAR CLEGG

DIRECTOR OF ENGINEERING

CERRITOS CA 90701

$714739-1991$

TRANSDUCERS INC

PETER R PERINO

PRESIDENT

14030 BOLSA LANE

CERRITOS CA 90701

$714739-1991$
TRANSDUCERS INCORPORATED

JOHN J FAHY

MARKET ING DIRECTOR

14030 BOLSA LN

CERRITOS, CA 90701

$714739-1991 \times 221$

TREE TOP INC

RANDALL F WILKINSON

DIRECTOR OF OUALITY ASSURANCE P 0 BOX 248

SELAH. WA 98942

$509697-7251 \times 621$

TRI-GAS E OIL CO INC

LEE D MCMAHAN

PRES IDENT

$P$ BOX 465

FEDERAL SBURG. MD 21632

$301754-8184$

TRIANGLE MACHINERY CO

WILLIAM H ZUSE

DIRECTOR MARKETING SERVICES

6655 WEST DIVERSEY AVE

CHICAGO, IL 60635

$312889-0200$

TRISTATE GRAIN INSPECTION SVC DAMON SAMPSON

WEI GHMAS TER

3906 RIVER ROAD

CINCINNATI, OH 45204

$513251-6571$

TROEMNER HENRY INC

WILBERT D ABELE GENERAL MGR

6825 GREENHAY AVE

PHILADELPHIA. PA 19143

$215724-0800$

TROPICANA PRODUCTS INC

GORDON D HARTMAN

DIRECTOR CORP QUAL ITY CONTROL

P O BOX 338

BRADENTON. FL 33506

$813747-4461 \times 3220$

TRUCK-L ITE CO INC

THEODORE $R$ ANJOS

OEM SALES MANAGER

310 EAST ELMHODD AVE

FALONFR, NY 14733

$716665-6214 \times 261$

TURNER SCALE SERVICE

JAMES H TURNER

581-B GEORGE TODD DR

MONTGOMERY AL 36117

$205271-3232$

TWO SHELL PLAZA

HALTER TKACHUK

SHELL OIL COMPANY

777 HALKER ST/TSP ROOM 1142

HOUSTON. TX 7700

713 241-0502

U S BORAX RESEARCH CORP

SCOTT GR IFF IN

MANAGER CONSUMER PRODUCTS RES

412 CRESCENT HAY

ANAHE IM. CA 92801

$714774-2670$

U S METRIC ASSOCIATION

LOUIS F SOKOI

PRESIDENT EMER ITUS

SUGARLOAF STAR RT

BOULDER, CO 80302

$303442-3342$ 
U S SUZUKI MOTOR CORPORATION FREDERIC M PETLER

HEAD ADMINISTRATION

3251 EAST IMPER IAL HWY

BREA, CA 92621

$714996-7040 \times 278$

U S TOBACCO CO

JOHN T CRAMBES

1I6OI COPENHAGEN COURT

FRANKLIN PARK, IL 60131

$312595-8200$

UNC NAVAL PRODUCIS

JAMES C SCHWARTZ

PROJECT LEADER

67 SANDY DESERT RD

UNCASVILLE, CT 06382

$203848-1511 \times 2482$

UNGAR CONSULTATION SERVICES

DAN UNG AR

CONSULTANT

30 LAFA YETTE DR

WOODMERE NY 11598

$516374-5690$

UNIDYNAMICS/ST LOUIS INC

THOM HUELLINGHORST

ENG INEER ING MANAGER

1326 ASHBY RD

ST LOUIS. MO 63132

$314991-0240$

UNIDN CARBIDE CDRP LINDE DIV RICHARD M HAYFORD

CONSUL T ANT

PO BOX 44

TONAWANDA, NY 14151

$716879-7207$

UNION CARBIDE CORPORATION

JOHN J HENKE

$P O B O X 2831$ BLDG 8280

CHARLESTON, WV 25330

$304747-6200$

UNION CARBIDE H $\&$ A DIVISION ALAN F ROGERS

MANAGER QUALITY ASSURANCE

88 LONG HILL STREET

EAST HARTFORD, CT 06108

$203728-6086 \times 350$

UNION OIL OF CALIFORNIA

OP E KITCHEN

$P$ O BOX 7600

LOS ANGELES: CA 90051

UNION PACIFIC RAILROAD

G J MEYER

1416 DODGE ST ROOM 1000

1416 DODGE ST ROOM 1026

OMAHA NE 68179

$402271-3153$

UNITED AIR LINES SFOQA

JOHN H SULLIVAN

METROLOGY SUPVSR

INTL AIRPORT

SAN FRANCISCO, CA 94128

$415876-4780$

UN I TED AMER ICAN FUELS

DALE L GREENBANK

SUPPLY \& DISTRIBUTION MGR

$P$ O BOX 16627

PHOENIX. AZ 85011

$602253-8981$
UNITED SCALE CORP INC

RONALD A THOMAS

PRESIDENT

$P 0$ BOX 376

CEDAR BLUFF, VA 24609

$703963-0343$

UNITED STANDARDS LAB INC

FRANK A DOLEZAL

PRE S I DENT

CAL IBRATION SVC-4250 NICOLLET

MINNEAPOLIS. MN 55409

$612823-4438$

UNITED STATES STEEL CORP

DAVID K JOHNSTONE

TRAFFIC AND TRANS ENGINEER

600 GRANT STREET RM 460

PITTSBURGH, PA 15230

$412433-3075$

UNI VERSAL COOPERATIVES INC

LOYAL W LEITGEN

MANAGER - THINE DEPT

7801 METRO PARKWAY

MINNEAPOLIS. MN 55420

$612854-0800 \times 182$

UNIVERSEL EPSCO INC

RUTHERFORD L ELLIS JR

VICE PRESIDENT-ELECTRONICS

1494 ELLSWORTH IND DR $8 \times 93544$

ATLANTA, GA 30318

$404351-2740$

US BORAX

JOHN M MANN

SEN IOR COUNSEL

3075 WILSHIRE BLVD

LOS ANGELES. CA 90010

$213381-5311 \times 1534$

USDA AGR ICULTURAL MARKETING SE STEPHEN RAYNER

STAFF OFFICER

$14 \mathrm{TH} \&$ INDEPENDENCE RM $0709 \mathrm{~S}$

ASHINGTON. DC 20250

$202447-5021$

UTAH INTERNATIONAL NAVAJO MINE R L GATES

PLANT SUPERINTENDENT

$P$ O BOX 155

FRUITLAND. NM 87416

$505598-5861$

VALLFY SCALE COMPANY

GLENN W HAYES

PRESIDENT

2306 h' BURBANK BLVD

BURBANK. CA 91506

$213841-0151$

VAN DYKE SCALE CO

GEORGE J BARBER

SCALE TECHNITION

2825 RAYTOWN RD

KANSAS CITY. MO 64128

$816861-7191$

VAN DYKE SCALE CO

ROGER HOWELL

SCALE TECHNICION

2825 RAYTOWN RD

KANSAS CITY, MO 64128

$816861-7191 \times 92$

VAN DYKE SCALE CO

GEORGE VANDYKE

OWNER

2825 RAYIOWN RD

KANSAS CITY. MO 64128 
YAN DYKE SCALE INC RONALO WEESE 2825 RAYTOHN RD
KANSAS CITY, MO 64128
$816861-7191$

VANDE BERG SCALES DON L VANDE BERG

OWNER

770 7TH ST NH IA 51250

SI QUX CENTER

VEEDER ROOT CO

RICHARD L SEITL
MGR HECH PRODUCT ENGR

28 SARGEANT ST

HATFORO: CT 06102
$203527-7201 \times 430$

VEEDER-ROOT CO

SAMUEL O COEN

SENIOR RESIDENT ENGINEER

PO BOX 1673

ALTOONA: PA 16603

$814695-4476 \times 78$

VEEDER-ROOT CO

ALFRED C EVANS $V$ VICE PRESIDENT ENGI NEERING

VICE PRESIDENT
70 SARGEANT ST

HARTFORD, CT 06102

203 527-7201

VEEDER-ROOT COMPANY

DONALD E MILLS MANAGER

PO BOX 1673

ALTOONA: PA 16603

$814695-4476 \times 25$

VEGA VEKT A/S

MECH ENG

$P$ O BOX $158 \quad N-1360$

NESBRU

VENTURE PACKAGING

PRESTON B BRYANT

ART DIRECTOR

PO BOX 7149

CHARLOT TE: NC 28217

VIOAL SASSOON INC

JOHN A MAC INTOSH

9640 OWENSMOUTH AVE

CHATWORTH, CA 91311

VIKING FEEDS INC

HERB N FLOAN

MANAGER

305 H GRANT ST/PO BOX 804

DETROIT LAKES, MN 56501

$218847-4749$

VINCE HAGAN CO

B D RAY

VICE PRESIDENT ENGINEERING

PO BOX 225141

DALLAS. IX 75265

$214339-7194$

H E T A VERY LTO

$G$ F HOD SMAN

LEGAL METROLOGY EXECUTIVE SME THWICK WARLEY

HEST MI DLANDS

0213537693
PATLEE BURPEE COMPANY

MANAGER PACKAG ING

300 PARK AYE

WARMINSTER: PA 18974

$215^{2} 674-4900^{\circ} \times 255$

H M BARR \& CO

RICK E MILLER

QUALI TY CONTROL SUPERVISOR

2105 CHANNEL AVE

MEMPHIS. TN 38101

WAKEFERN FOOD CORP

MYRON A SCHMUTZER

VICE PRES QUALITY ASSURANCE

600 YORK ST

ELI ZABETH, NJ 07207

$201527-3422$

HALGREEN LABS INC

$T S$ SUN

MGR OUAL CONTROL/ASSURANCE

3308 CQVINGTON ROAD

KAL AMAZOO, MI 49002

$616 \quad 349-1096$

WALNUT GROVE PRODUCTS

JERRY OVERTON

MANAGER OF OUALITY CONTROL

SECOND AND LINN

ATLANTIC I I A 50022

WARNER LAMBERT PHARMACEUTICAL $R C$ ERNST

201 TABOR RD

MORRIS PLAINS. NJ 07950

WAYNE DIV-DRESSER INDUSTRIES

LARRY MURRAY

CHIEF ENGINEER

$124 \mathrm{~W}$ COLLEGE AVE PO BOX 1859

SAL ISBURY, MD 21801

301 546-6690

WEBB CORPORATION

R ICHARD BENTLEY

MANAGER SCALE DEPT

$P O B O X \quad 549$

HEBB CITY: MO 64870

$417673-4646$

WEBBER OIL COMPANY

LEON L ST LOUIS

MAINTENANCE SUPER VISOR

700 MAIN ST

BANGOR, ME 04401

$207942-5501 \times 276$

HEBSTER INDUSTRIES INC

WILLIAM J CRISAFI

DIRECTOR OF MANUFACTURING

58 PULASKI ST

PEABODY, MA 01960

$617532-2000$

WEBTER SCALE SALES AND SERVICE

ROGER E SHOEMAKER

PAR TNER

PO BOX 127

WEBTER , SD 57274

$605345-3881$

HEI GH-TRONIX I NC

RICHARD S BRADLEY

PRESIDEN I

1000 ARMSTRONG DR

FAIRMONT. MN 56031

$507238-4461 \times 200$ 
WEIGHING 6 AUTOMATION SYSTEMS SALYATORE A BARBERA

PRESIDENT

20 PASSAIC ST

$201777-552207026$

HEIGHING \& CONTROL SYSTEMS INC DANIEL \& COCKRELL

PRESIDENT

P 0 BOX 1483

BRANDON. FI 33511

HEIGHTS \& MEASURES CCAC

C HAHKINS

GRAYIMETRIC SPECIALIST

4900 YUNGE ST-SUITE 601

HLLOHDALE M2N6B 8

HEIGHTS AND MEASURES CONTROL

DONALO 1 LYNCH

STANDARDS ADMINISTRATOR

710 NORTH 17 STREET

KANSAS CITY KS 66102
913 342-5243

MEIGHTS AND MEASURES SERVICE

GEORGE P TSIARTZAZIS

METRICATION OFFICER

NICOSIA

$4034413 \times 24$

HELD-IT COMPANY

SHELDON SCHAFFER

4477 SHEILA ST

LOS ANGELES, CA 90023

WELOOTRON CORP

HILLIAM NOHR

MGR FOOD PACKAGING SERVICE

1532 S HASHINGTON AVE AT I-287

$201752-6780 \times 372$

HEST POINT-PEPPERELL INC

BARRY F SHEA

ASSOC LEGAL COUNSEL/ASST SECY

PO BOX 71

HEST POINT GA 31833

HEST 10 TH ST

MENE L ARNETT

ANNISTON AL 36201

$205236-6381$

HESTERN FUELS ASSOCIATION INC ROD $J$ HOLF

WYOMING OPERATIONS SUPERVISOR

203 CAREY AVE

GILLETTE HY 82716

HESTERN HEIGHING \& INSP BUR

$R$ D HEER

MANAGER-TRANSIT G AUDIT SERV

222 S RIVERSIDE PLAZA RM 1130

CHICAGO IL 60606

312 559-0510

HESTERN WEIGHING \& INSP BUREAU

DHAYNE E CIRCLE

CHIEF TRAVELING AGENT

3435 BROADWAY SUITE 201

KANSAS CITY, MO 64111

$816753-2101 \times 17$

HESTINGHOUS AESD

HILL AM P THIGG

SENIOR ENG INEER

PO BOX 746 MAIL STOP 550

BALTIMORE MO 21203

$301765-3606$
HHITES PETROLEUM SERVICE

RAYMOND H HHITE

OWNER

385 ARNDT ST PO BOX 1241

$414922-393054935$

HIDE RANGE SCALE SERVICE

MICHAEL HERMES

OWNER

991 BEARS DEN RD

YOUNGSTOWN $\mathrm{OH} 44511$

HIDGEON ENTERPRISES INC DAVIDP HIDGEON

PRESIDENT

ROUTE 13 SOUTH P 0 BOX 8

LAUREL DE 19956

उO2 $875-5777$

HILKINSON SCALE COMPANY INC GEORGE D WILKINSON

PRE S I DENT

8800 WILKINSON BLVD

CHARLOTTE, NC 2821

$704394-1392 \times 385$

HILLIAM M HILSONS SONS INC

CHARLES J DENNY 8 \& VALLEY FORGE RDS

LANSDALE, PA 19446

$215855-4631 \times 37$

WILLIAM PIKE COMPANY

RICHARD W THOMPSON

GENERAL MANAGER-SECRETARY

7741 DIX AVE

DEIROIT. MI 48209

$313849-1300$

WILLIAMS PETROLEUM SVC E EOUIP

ROBERT R HILLIANS

OUNER

RR $680 \times 328$

BEMIDJI. MN 56601

$218586-2038$

WINSLOW SCALE COMPANY

JOSEPH GINER

PRESIDENT

PO BOX 1523

TERRE HAUTE, IN 47808

WISCONSIN ELECTRICAL MFG CO DONALD HAGENBAR

VICE PRESIDENT ENGINEER ING

2501 SOUTH MOORLANO ROAD

NEW BERLIN. WI 53151

$424782=2340$

HM DIXON CO

JAMES J MANNING

PURCHASING AGENT

750 HASHINGTON AVE

CARLSTADT, NJ 07072

$201939-6700$

HORLO WIDE MEIGHING

GIENN J DOUGLAS

DIRECTOR OF MARKETING

$11863124 \mathrm{TH}$ AVE NE

KIRKLAND. HA 98034

WYANDOT INC

JACKIE CAPL INGER

OUALITY ASSURANCE MANAGER

135 HYANDOT AVE

MARION, OH 43302

$614383-4031$

2 R OIL EQUIPAENT CO

ROBERT F IINDEL

PO BOX 38

OREANA. IL 62554 


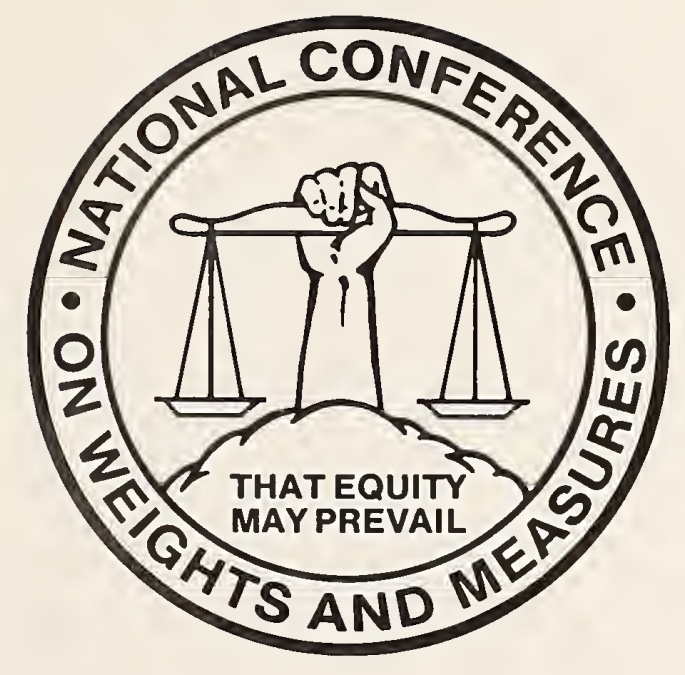

NCWM MEMBERSHIP APPLICATION

Please accepl my application for membership in THE NATIONAL CONFERENCE ON WEIGHTSAND MEASURES. My check made payable to "National Conference on Weights and Measures" is enclosed to cover the annual membership fee of $\$ 35.00$ for the period ending June 30. Mail to: P.O. Box 3137. Gaithersburg. MD 20878.

(Please fill in form completely -- abbreviate only if necessary)
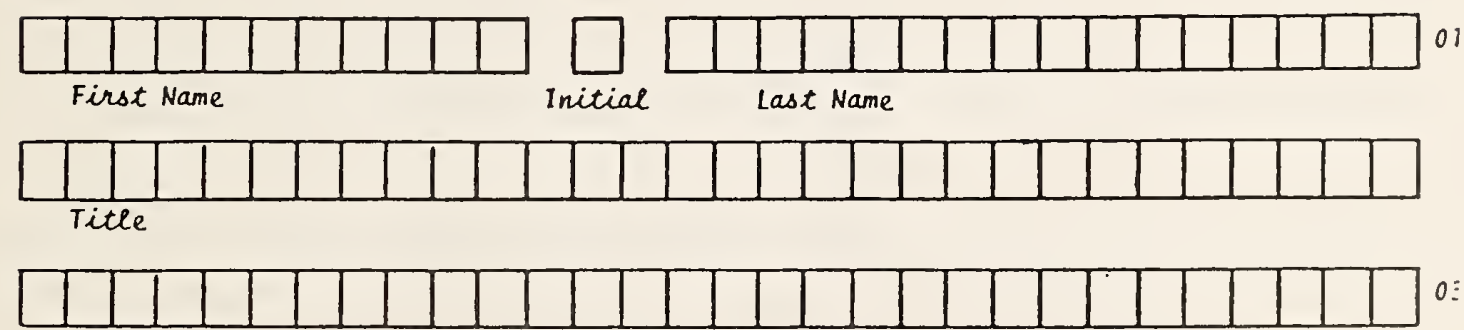

Organization.

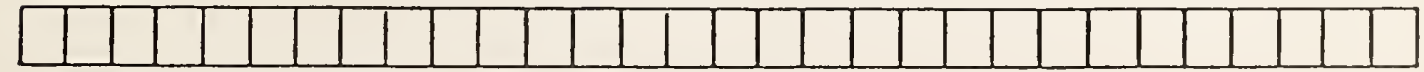

Business Street Address
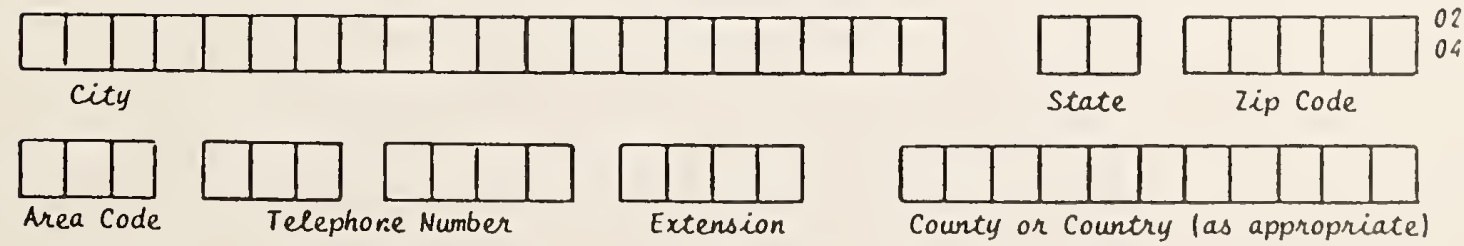




U.S. DEPARTMENT OF COMMERCE

National Bureau of Standards

Gaithersburg, MD 20899

OFFICIAL BUSINESS

Penalty for Private Use, $\$ 300$
SPECIAL FOURTH-CLASS RATE

POSTAGE \& FEES PAID

NBS

GAITHERSBURG, MD

PERMIT No. G195 